\title{
Uranium Oxide Aerosol Transport in Porous Graphite
}

\author{
J Blanchard \\ DC Gerlach \\ RD Scheele \\ ML Stewart \\ BD Reid \\ PA Gauglitz \\ LM Bagaasen
}

C Brown

C Lovin

$\mathrm{CH}$ Delegard

A Zelenyuk

EC Buck

BJ Riley

CA Burns

January 2012

Pacific Northwest

NATIONAL LABORATORY

Proudly Operated by Battelle Since 1965 


\title{
DISCLAIMER
}

This report was prepared as an account of work sponsored by an agency of the United States Government. Neither the United States Government nor any agency thereof, nor Battelle Memorial Institute, nor any of their employees, makes any warranty, express or implied, or assumes any legal liability or responsibility for the accuracy, completeness, or usefulness of any information, apparatus, product, or process disclosed, or represents that its use would not infringe privately owned rights. Reference herein to any specific commercial product, process, or service by trade name, trademark, manufacturer, or otherwise does not necessarily constitute or imply its endorsement, recommendation, or favoring by the United States Government or any agency thereof, or Battelle Memorial Institute. The views and opinions of authors expressed herein do not necessarily state or reflect those of the United States Government or any agency thereof.

\author{
PACIFIC NORTHWEST NATIONAL LABORATORY \\ operated by \\ BATTELLE \\ for the \\ UNITED STATES DEPARTMENT OF ENERGY \\ under Contract DE-AC05-76RL01830 \\ Printed in the United States of America \\ Available to DOE and DOE contractors from the \\ Office of Scientific and Technical Information, \\ P.O. Box 62, Oak Ridge, TN 37831-0062; \\ ph: (865) 576-8401 \\ fax: (865) 576-5728 \\ email: reports@adonis.osti.gov

\begin{abstract}
Available to the public from the National Technical Information Service, U.S. Department of Commerce, 5285 Port Royal Rd., Springfield, VA 22161 ph: (800) 553-6847 fax: (703) 605-6900 email: orders@ $@$ ntis.fedworld.gov online ordering: http://www.ntis.gov/ordering.htm
\end{abstract}




\title{
Uranium Oxide Aerosol Transport in Porous Graphite
}

\author{
J Blanchard \\ C Brown \\ DC Gerlach \\ C Lovin \\ RD Scheele \\ $\mathrm{CH}$ Delegard \\ ML Stewart \\ A Zelenyuk \\ BD Reid \\ EC Buck \\ PA Gauglitz \\ BJ Riley \\ LM Bagaasen \\ CA Burns
}

January 2012

Prepared for the U.S. Department of Energy under Contract DE-AC05-76RL01830

Pacific Northwest National Laboratory

Richland, Washington 99352 


\section{Summary}

Conditions exist in carbon dioxide $\left(\mathrm{CO}_{2}\right)$ gas-cooled, graphite moderated reactors that might allow for gaseous transport of uranium oxide $\left(\mathrm{UO}_{2}\right)$ particles into the graphite moderator. The transport of $\mathrm{UO}_{2}$ in the reactor coolant system, and subsequent deposition of this material in the graphite, is of interest due to the potential to influence the application of the Graphite Isotope Ratio Method (GIRM). GIRM was developed to validate the declared operation of graphite moderated reactors.

Uranium impurities in nuclear grade graphite are one of several possible indicator elements that can be used for a GIRM assessment. During fuel failures, uranium metal in the fuel is exposed to the $\mathrm{CO}_{2}$ coolant and oxidizes as either $\mathrm{UO}_{2}$ or $\mathrm{U}_{3} \mathrm{O}_{8}$. Measurements in adjacent fuel channels indicate that $\mathrm{CO}_{2}$ coolant readily flows through the porous graphite moderator blocks. Therefore, the potential exists for the coolant gas to transport $\mathrm{UO}_{2}$ particles, as an aerosol, into the porous graphite, thereby invalidating uranium as an indicator element. Scoping calculations indicated that a mass of $\mathrm{UO}_{2}$ particles sufficient to impact typical background levels in the graphite could be produced during the life of a graphite reactor. Air flow velocities in these reactors were more than sufficient to transport $\mathrm{UO}_{2}$ particles to channels adjacent to where the fuel failure occurred.

The objective of the work, summarized in this report, was to address the feasibility and extent of $\mathrm{UO}_{2}$ particle transport in porous graphite. Using a theoretical model, based on classical aerosol filtration, a conservative, worst-case, particle size distribution (PSD) was determined. Experiments were then designed to evaluate the aerosol flow, using the worst case PSD, through well-characterized porous graphite samples. The graphite samples were analyzed to determine the $\mathrm{UO}_{2}$ concentration profile and quantify the depth of penetration. The experimental results were also compared to model data to further understand the physics of $\mathrm{UO}_{2}$ aerosol flow in porous graphite.

The graphite penetration tests indicate that it is possible for uranium from fuel failures to be transported by the $\mathrm{CO}_{2}$ gas coolant system and be deposited at depths as great as $35 \mathrm{~mm}$ in the graphite blocks of a graphite moderated reactor. Since a nominal GIRM graphite sample is taken within the first $19 \mathrm{~mm}$, uranium from fuel failures has the potential to adversely impact a GIRM assessment.

The penetration of uranium seen in these worst case tests may not be realized when $\mathrm{UO}_{2}$ is generated from fuel failures in an actual reactor environment. Nonetheless, these tests show that steps must be taken to identify and ensure that uranium contamination from fuel failures does not adversely impact a GIRM assessment. A standard protocol to evaluate whether a GIRM graphite sample has been compromised by uranium from fuel failures should be implemented and appropriate steps should be taken to ensure that any compromised sample uses other indicator elements for GIRM assessment. 



\section{Table of Contents}

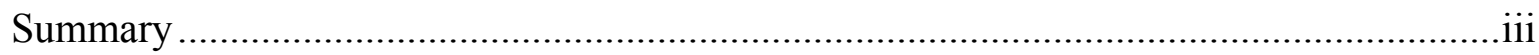

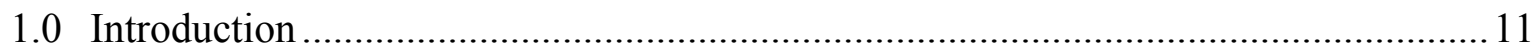

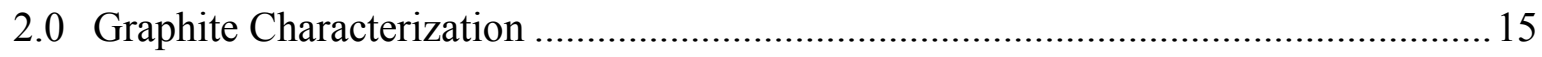

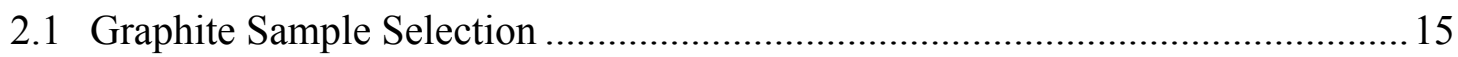

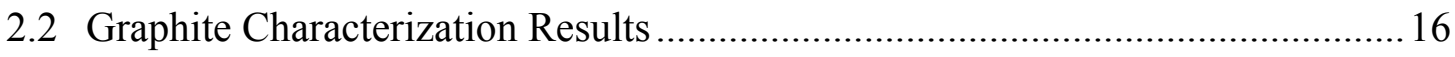

2.3 Graphite Uranium Background Content......................................................... 19

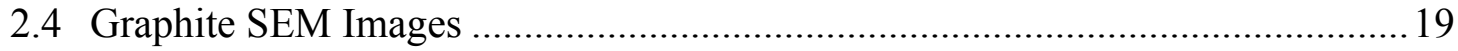

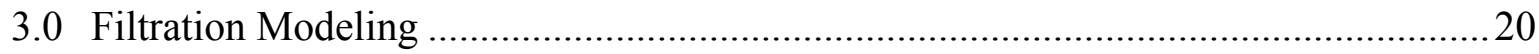

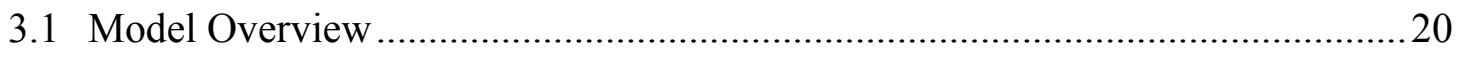

3.2 Conservative Aerosol Particle Size Estimation ………….................................... 21

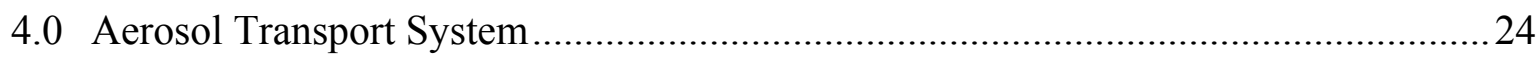

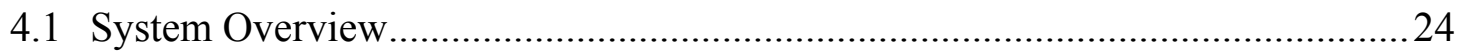

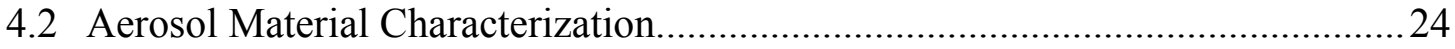

4.2.1 Nature of Uranium Oxide Produced by $\mathrm{CO}_{2}$ Oxidation .............................24

4.2.2 Carbon Dioxide Oxidation of Uranium.....................................................25

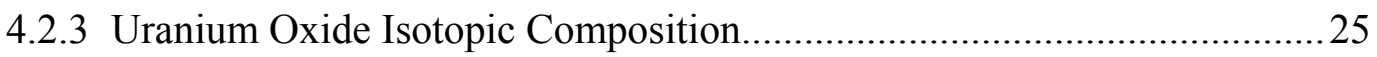

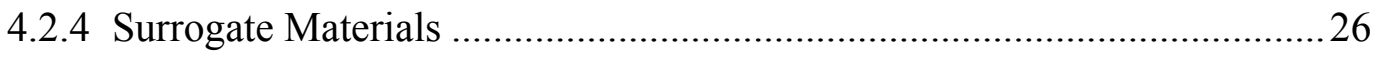

4.3 Phase I: Surrogate Aerosol Experimental Apparatus ………………................28

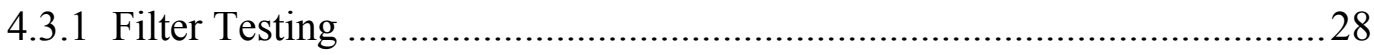

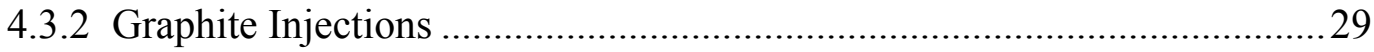

4.4 Phase II: Uranium Oxide Aerosol Experimental Apparatus ………………….......30

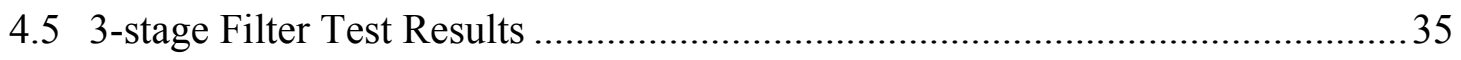

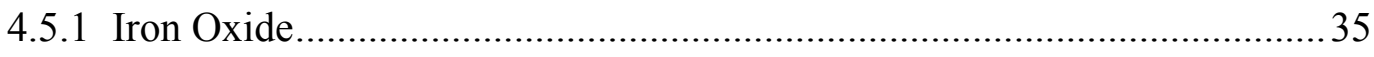

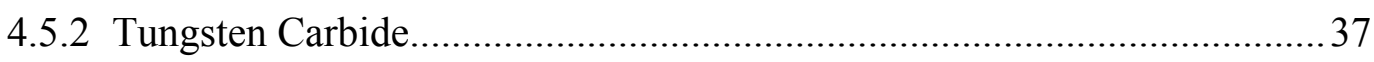

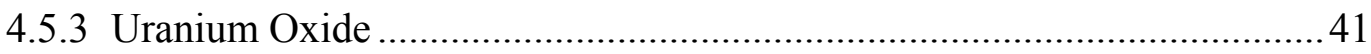

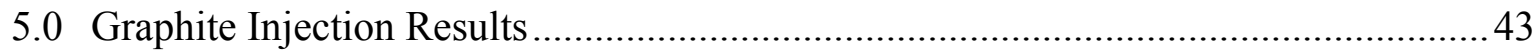

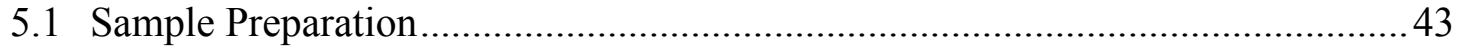

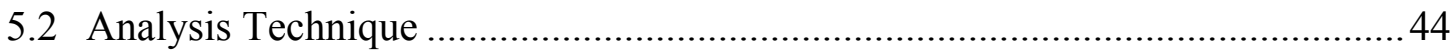

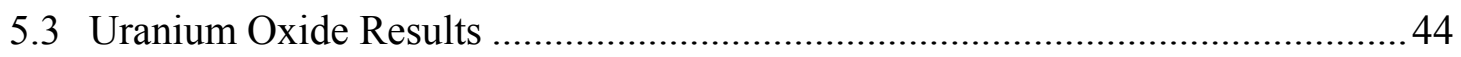

5.3.1 ATS Testing Data .............................................................................. 44

5.3.2 Tuned Unit Collector Model ...................................................................... 47

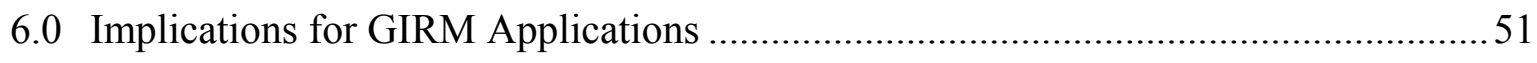

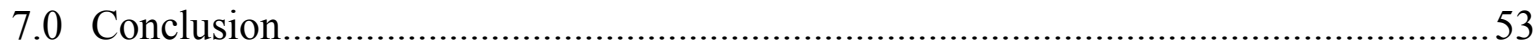

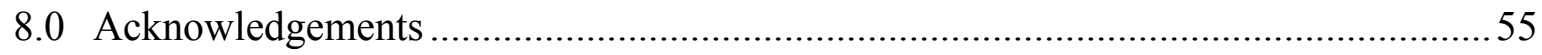


PNNL-21014

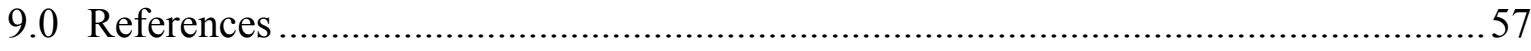

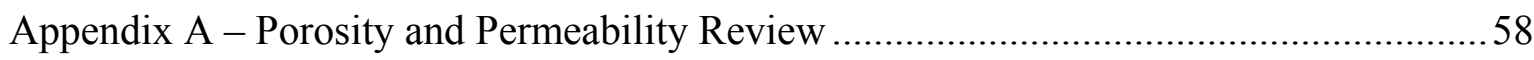

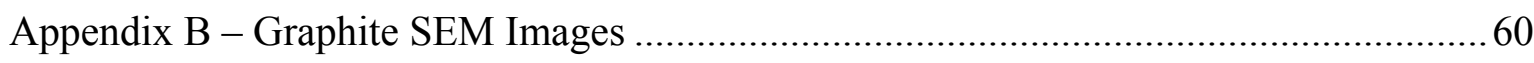

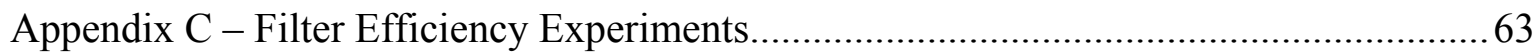

Appendix D - Aerosol Material SEM Images .............................................................. 70

Appendix E - Aerosol Transport System Information ....................................................76

Appendix F - Review of Carbon Dioxide Oxidation of Uranium........................................... 81

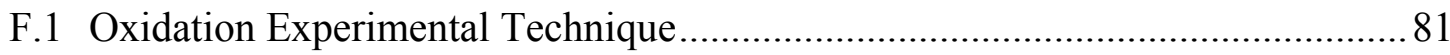

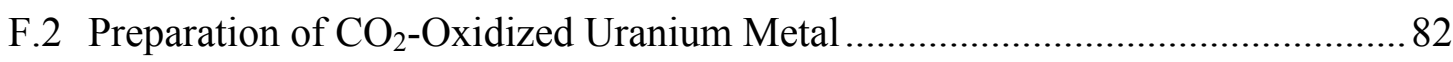

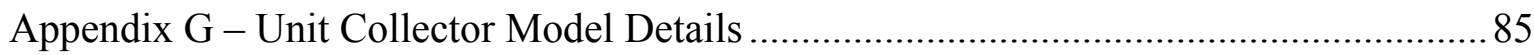




\section{List of Figures}

Figure 1: Schematic of exaggerated fuel failure scenario. 12

Figure 2: $\mathrm{UO}_{2}$ particle lofting capability by reactor plenum $\mathrm{CO}_{2}$ coolant flow. Coolant flow of $2 \mathrm{~m} / \mathrm{s}$ is typical in reactor plenums. Note SF defines the particle shape factor, or ratio of horizontal and vertical dimensions...

Figure 3: $\mathrm{UO}_{2}$ particle lofting capability by reactor fuel channel $\mathrm{CO}_{2}$ coolant flow. Coolant flow of $20 \mathrm{~m} / \mathrm{s}$ is typical in reactor fuel channels. Note SF defines the particle shape factor, or ratio of horizontal and vertical dimensions.

Figure 4: Potted graphite schematic.

Figure 5: Permeability regressions for PGX graphite sample 19. 16

Figure 6. Graphite characterization results for 17 PGX graphite samples. 17

Figure 7: Mercury porosimetry pore size distribution results for BR, CX, PGX and RM graphite, where the vertical axis shows the slope of the intruded volume per log pore diameter which provides a measure of the pore volume.

Figure 8: Mercury porosimetry cumulative pore size distribution results for BR, CX, PGX and RM graphite.

Figure 9: Several mechanisms leading to particle capture on a spherical unit collector. ....21

Figure 10: Estimation penetration depth as a function of particle size using unit collector model, assuming a face velocity of $3.75 \mathrm{~cm} / \mathrm{s}$.

Figure 11: Contributions of various capture mechanisms to total capture efficiency for $\mathrm{UO}_{2}$ particles.

Figure 12: High magnification SEM image of uranium oxide. Both fundamental particles

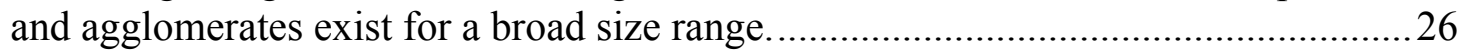

Figure 13: Iron oxide particle size distribution............................................................. 27

Figure 14: Tungsten carbide particle size distribution..............................................28

Figure 15: ATS 3-stage filter test configuration schematic.........................................29

Figure 16: ATS graphite injection test configuration schematic. ................................. 30

Figure 17: Radioactive environment ATS graphite injection test configuration schematic.34

Figure 18: Iron oxide filter test mass collected results. ................................................. 36

Figure 19: Iron oxide filter test percent collected results. ......................................... 37

Figure 20: Tungsten carbide filter test mass collected results. ..................................... 39

Figure 21: Tungsten carbide filter test percent collected results. .................................. 39

Figure 22: Iron oxide and tungsten carbide mass collected filter test comparison............. 40

Figure 23: Iron oxide and tungsten carbide percent collected filter test comparison......... 40

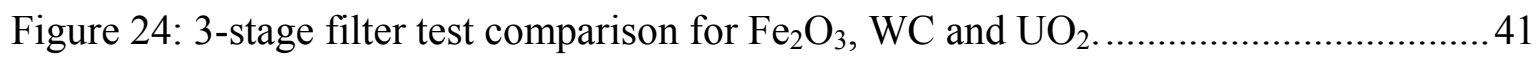

Figure 25: Graphite injection sample preparation and slicing protocol. Note that $\sim 2 \mathrm{~mm}$ were machined from the outer surface of the graphite prior to slicing operations....... 43 
Figure 26: $\mathrm{U}_{236}$ isotope concentration profile in graphite samples.

Figure 27: ATS experiment and unit collector model comparison of $\mathrm{UO}_{2}$ concentration profile.

Figure 28: Mass collected on $5 \mu \mathrm{m}$ filters in $\mathrm{UO}_{2}$ graphite injection and filter tests ......... 46

Figure 29: Unit collector model inlet mass fraction. ................................................... 48

Figure 30: Comparison of particle penetration predicted by the preliminary and tuned unit

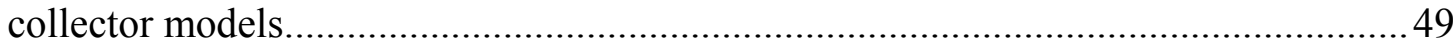

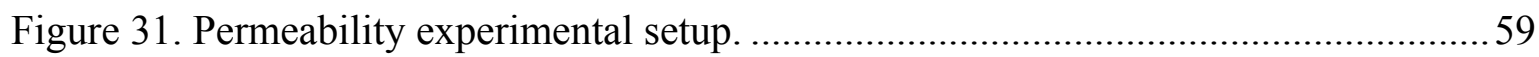

Figure 32: SEM images of PGX sample 17 inlet cross-section at $12 \mathrm{x}$ magnification. The

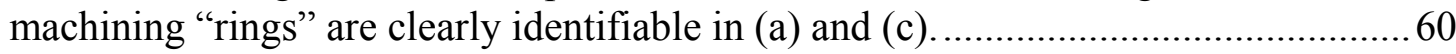

Figure 33: SEM images of PGX sample 17 inlet cross-section at 100x magnification. Pores ranging from $50-100 \mu \mathrm{m}$ can be seen in (a), (c), (d) and (e). A large 150 by $600 \mu \mathrm{m}$ pore is present in (b).

Figure 34: SEM images of PGX sample 17 inlet cross-section. The magnification of (a) and (b) is 500 and $1000 \mathrm{x}$, respectively.

Figure 35: Schematic and photographs of filter efficiency experimental setup...............64

Figure 36: Filter efficiency experiment results for graphite sample FEE21.....................65

Figure 37: Expected profile of filter efficiency versus particle size...............................66

Figure 38: Filter efficiency data for $40 \mathrm{~nm}$ particles where (a) and (b) is measured at the

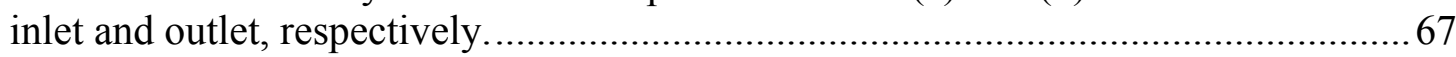

Figure 39: Filter efficiency data for $100 \mathrm{~nm}$ particles where (a) and (b) is measured at the inlet and outlet, respectively.

Figure 40: Filter efficiency data for $200 \mathrm{~nm}$ particles where (a) and (b) is measured at the inlet and outlet, respectively.

Figure 41: Filter efficiency data for $300 \mathrm{~nm}$ particles where (a) and (b) is measured at the inlet and outlet, respectively.

Figure 42: Filter efficiency data for $400 \mathrm{~nm}$ particles where (a) and (b) is measured at the inlet and outlet, respectively.

Figure 43: SEM images of iron oxide. Micrographs (A), (B) and (C) were captured at $5,000 \mathrm{X}, 10,000 \mathrm{X}$ and 20,000X, respectively.

Figure 44: SEM images of tungsten carbide. Micrographs (A), (B) and (C) were captured at 5,000X, 10,000X and 20,000X, respectively.

Figure 45: SEM image of uranium oxide. Note many large agglomerates are present. ...... 72

Figure 46: SEM image of uranium oxide showing small order $200 \mathrm{~nm}$ particles affixed to the surface of large order $20 \mu \mathrm{m}$ particles and agglomerates.................................... 73

Figure 47: SEM image of uranium oxide showing many small particles........................ 74

Figure 48: High magnification SEM image of uranium oxide. Extremely small particles can be seen on the surface of large agglomerates................................................. 75

Figure 49: Comparison of Nitrogen and Carbon Dioxide gas properties. ........................ 76 
Figure 50: Photographs of ATS components. 3-stage filters mounted in vacuum fittings are shown in (a) and (b). A loading tee is shown in (b) and mounted in downstream of the isolation valve in (c). A sample $5 \mu \mathrm{m}$ pre-filter with tungsten carbide is shown in (d)

Figure 51: Photograph of potted graphite with pre-filter (a) and 3-stage filters (b)...........78

Figure 52: Photograph of Phase I ATS 79

Figure 53: Photograph of radiological environment ATS, where 1 is the pressure chamber, 2 is the loading tee, 3 is the mixing chamber, 4 is the $5 \mu \mathrm{m}$ pre-filter, 5 is the potted graphite sample and 6 is the pressure and flow measurement instruments. 80

Figure 54: Uranium metal beads, where the scale is in $\mathrm{cm}$ (Delegard et al. 2004). ........... 82

Figure 55: Particle size distribution of uranium metal beads (Delegard et al. 2004). .........82

Figure 56: Observed oxidation behavior of $\mathrm{U}$ metal in TG/DTA experiments ....................83

Figure 57: Continued $\mathrm{CO}_{2}$ oxidation of $\mathrm{U}$ metal experiment isothermally at $580^{\circ} \mathrm{C} \ldots \ldots \ldots . .84$ 


\section{List of Tables}

Table 1: Average Background Uranium content in three PGX graphite samples. ............. 19

Table 2: Unit Collector Model Parameters ...................................................................... 22

Table 3: Redding EU isotopic composition data. ........................................................25

Table 4: Characteristics of aerosol materials tested using ATS. .................................... 27

Table 5: Identification of the radioactive environment ATS components..........................33

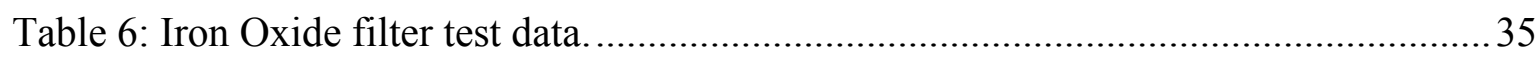

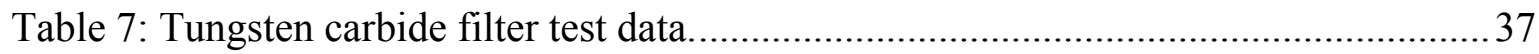

Table 8: Uranium Oxide filter test data. .................................................................... 41

Table 9: Concentration of material homogeneously distributed in a $5 \mathrm{~cm}$ thick graphite

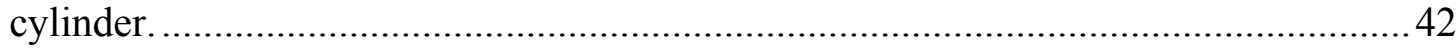

Table 10: Graphite injection sample nominal slicing dimensions. .................................. 43

Table 11: $\mathrm{UO}_{2}$ injection graphite permeability and flow rate summary .......................... 45

Table 12: Summary of $\mathrm{UO}_{2}$ mass measurements in filter and graphite tests.................... 47

Table 13: ATS 3-stage testing filter specifications.................................................... 76

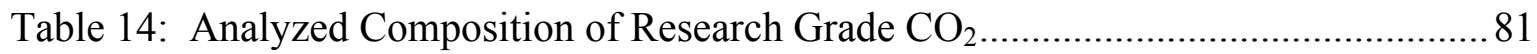


PNNL-21014

\subsection{Introduction}

Conditions exist in $\mathrm{CO}_{2}$ gas-cooled, graphite moderated reactors that might allow for gaseous transport of uranium oxide particles into the graphite moderator. The transport of uranium oxide in the reactor coolant system, and subsequent deposition of this material in the graphite, is of interest due to the potential to influence the application of the Graphite Isotope Ratio Method (GIRM). GIRM was developed to validate the declared operation of graphite moderated reactors. GIRM exploits isotopic ratio changes that occur in the impurity elements present in the graphite to infer cumulative exposure and hence the reactor's lifetime cumulative plutonium production. Refer to Gesh, et. al. (2004), for a more complete discussion on the GIRM technology.

Uranium impurities in nuclear grade graphite are one of several possible indicator elements that can be used for a GIRM assessment. Successful GIRM predictions require that the indicator element is present from the initial reactor core irradiation. If uranium is introduced into the graphite after the initial core irradiation, such as from a fuel failure during operation, there is a potential to create a bias in the GIRM results. Therefore, it is desirable to measure GIRM graphite samples that have not been contaminated with uranium introduced from fuel failures or other sources.

During fuel failures (e.g., a cladding breach) uranium metal in the fuel is exposed to the $\mathrm{CO}_{2}$ coolant. The uranium metal reacts with $\mathrm{CO}_{2}$ to form uranium oxide, as either $\mathrm{UO}_{2}$ or $\mathrm{U}_{3} \mathrm{O}_{8}$. The uranium oxide can also form through combination with the oxygen that is present in the coolant at around $10 \mathrm{ppm}$ due to radiolytic decomposition of $\mathrm{CO}_{2}$. The oxygen is more reactive with the uranium metal than $\mathrm{CO}_{2}$.

Measurements in adjacent fuel channels indicate that $\mathrm{CO}_{2}$ coolant readily flows through the porous graphite moderator blocks. This flow may be driven by as much as a 10 psig pressure gradient from the inner fuel channel to the outer surface of the graphite block. Therefore, the potential exists for the coolant gas to transport uranium oxide particles, as an aerosol, into the porous graphite, thereby invalidating uranium as an indicator element. This scenario is presented schematically in Figure 1, where the fuel failure has been accentuated for clarity. The potential concern is not associated with fuel failure channels because those channels can be avoided. The GIRM assessment could be affected, however, by the other channels, as indicated by the left side of the graphic, where fuel failure generated $\mathrm{UO}_{2}$ is circulated through the closed-loop system, enters other channels and is captured by the graphite. 
PNNL-21014

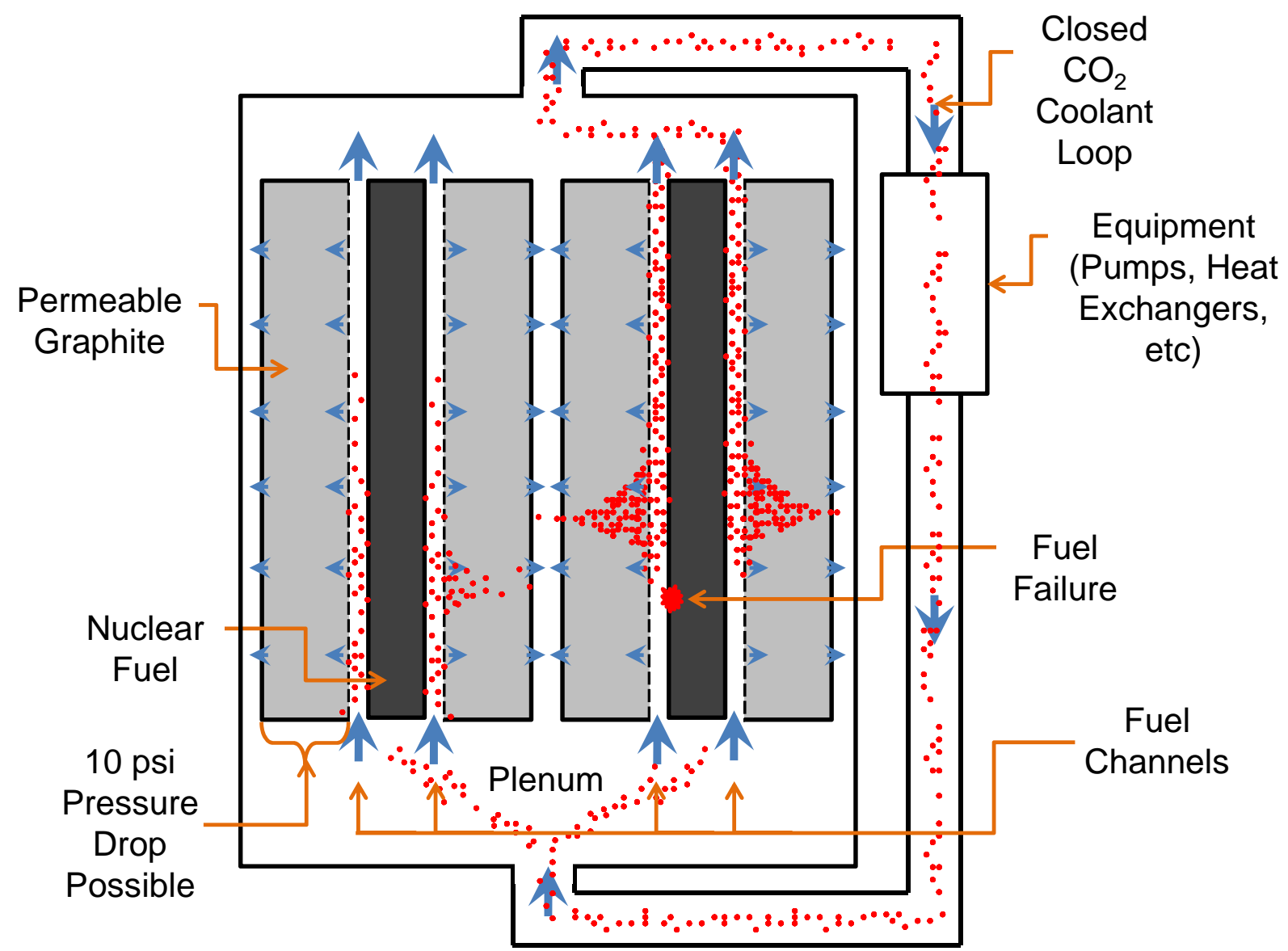

Figure 1: Schematic of exaggerated fuel failure scenario.

The amount of $\mathrm{UO}_{2}$ that might be generated from fuel failures can be approximated by assuming a $3.3 \mathrm{~cm}$ long by $0.3 \mathrm{~cm}$ wide split in the uranium cladding, exposing a $1 \mathrm{~cm}^{2}$ area. Furthermore, assuming the nuclear reactor core experiences 100 fuel failures over its lifetime and operates for 2 weeks after each failure, approximately $0.34 \mathrm{~g}$ of $\mathrm{UO}_{2}$ would be produced using an assumed oxidation rate of $0.01 \mathrm{mg} / \mathrm{cm}^{2}$ $\mathrm{hr}$ at $300{ }^{\circ} \mathrm{C}$. If $\mathrm{UO}_{2}$ were uniformly distributed in 500 metric tons of graphite, the uranium concentration from fuel failures would be $0.7 \mathrm{ppb}$. Typical uranium impurity levels in graphite are $10 \mathrm{ppb}$. Uranium from failed fuel at $10 \%$ of the impurity levels is a concern so the levels of $\mathrm{UO}_{2}$ that could be generated from fuel failures would be a concern especially when likely concentration effects near the channel wall are considered.

Additionally, settling theory can help determine the ability of the $\mathrm{CO}_{2}$ coolant stream to suspend and transport $\mathrm{UO}_{2}$ particles. The influence of particle size on the suspension potential by the plenum and 
channel gas flow is shown in Figure 2 and

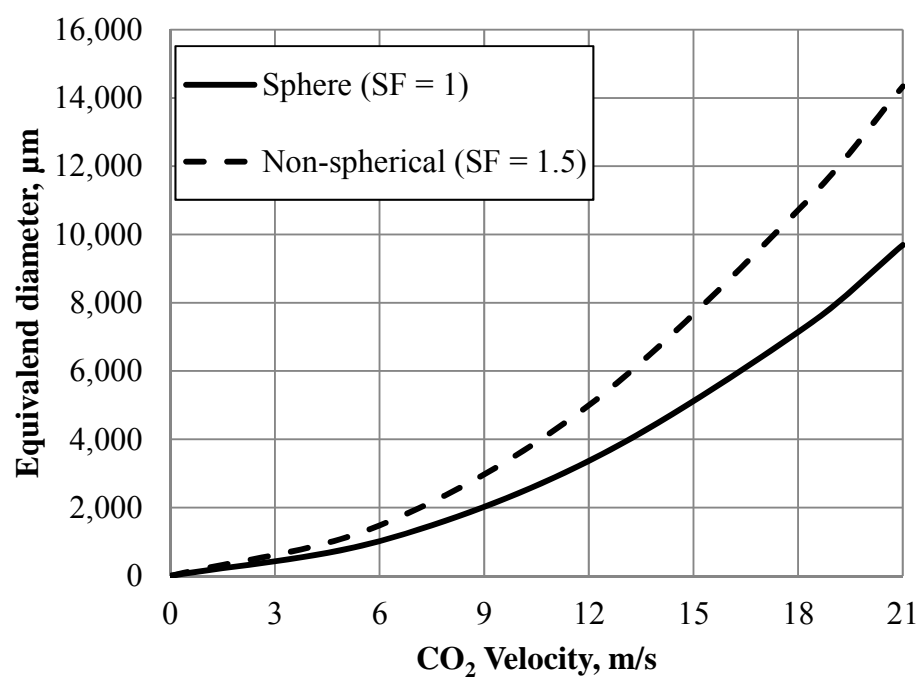

Figure 3, indicating it is easier to suspend smaller particles. Assuming the plenum and channel gas coolant flow rate is 2 and $20 \mathrm{~m} / \mathrm{s}$, respectively, spherical $\mathrm{UO}_{2}$ particles as large as $200 \mu \mathrm{m}$ in the plenum and $9 \mathrm{~mm}$ in a channel could be suspended (or lofted). Even if the $\mathrm{UO}_{2}$ particles that form are very large, size reduction as the particles flow through gas circulators, heat exchangers and other reactor components, is very likely.

The objective of this work was to address the feasibility and extent of $\mathrm{UO}_{2}$ particle transport in porous graphite. Using a theoretical model, based on classical aerosol filtration, a conservative, worst-case, particle size distribution (PSD) was determined. Experiments were then designed to evaluate worst-case PSD aerosol flow through well-characterized porous graphite samples. The graphite samples were analyzed to determine the $\mathrm{UO}_{2}$ concentration profile and quantify the depth of penetration. The experimental results were also compared to model data to further understand the physics of $\mathrm{UO}_{2}$ aerosol flow in porous graphite. 
PNNL-21014

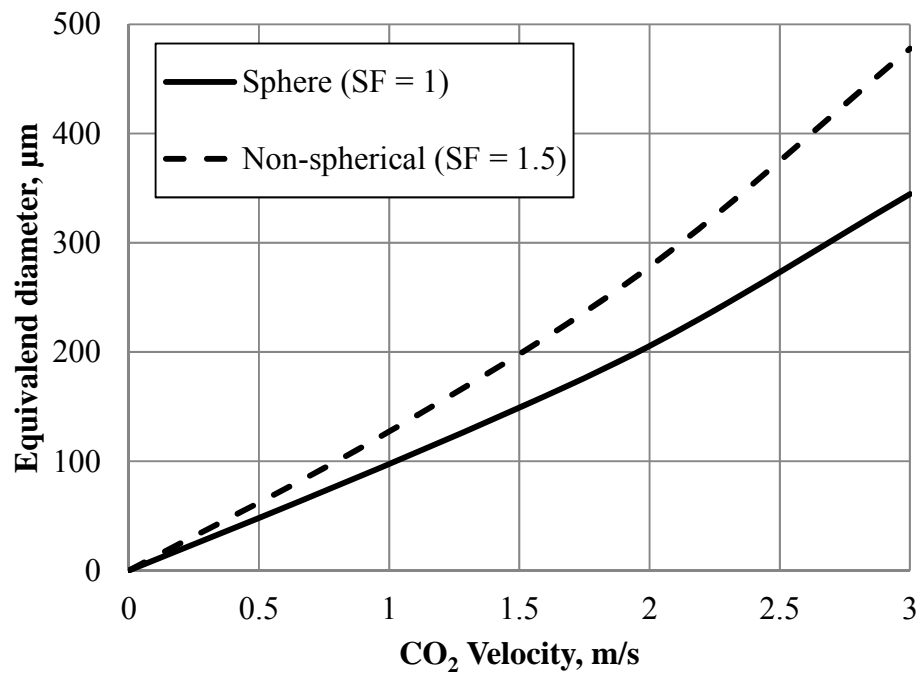

Figure 2: $\mathrm{UO}_{2}$ particle lofting capability by reactor plenum $\mathrm{CO}_{2}$ coolant flow. Coolant flow of $2 \mathrm{~m} / \mathrm{s}$ is typical in reactor plenums. Note SF defines the particle shape factor, or ratio of horizontal and vertical dimensions.

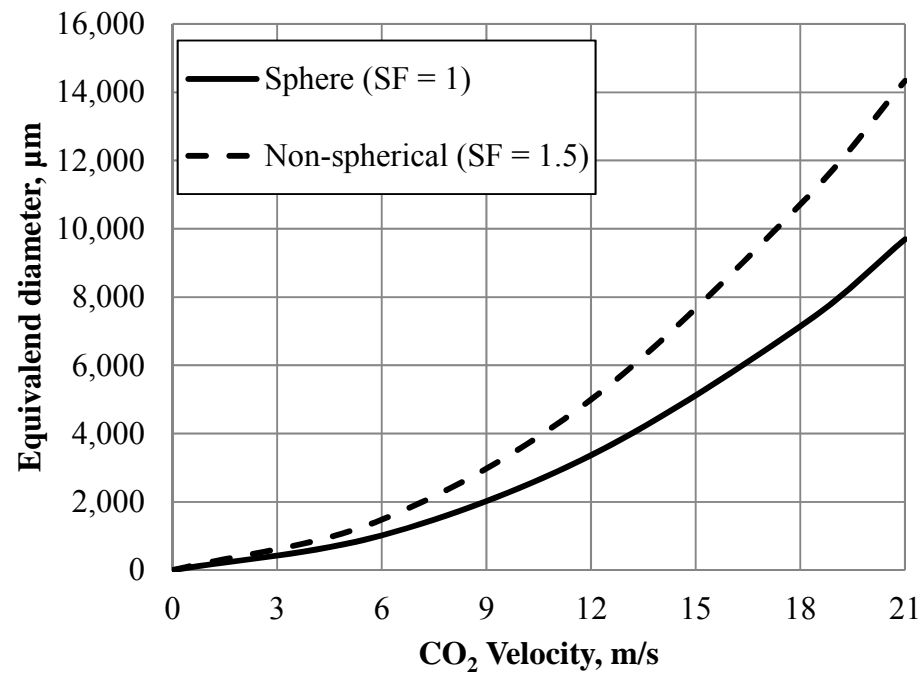

Figure 3: $\mathrm{UO}_{2}$ particle lofting capability by reactor fuel channel $\mathrm{CO}_{2}$ coolant flow. Coolant flow of $20 \mathrm{~m} / \mathrm{s}$ is typical in reactor fuel channels. Note SF defines the particle shape factor, or ratio of horizontal and vertical dimensions. 


\subsection{Graphite Characterization}

\subsection{Graphite Sample Selection}

Small graphite plugs were core drilled from larger blocks to act as test samples and mimic the expected flow path of the aerosol in typical reactor graphite blocks. The thickness of reactor graphite blocks, from the inner fuel channel to the outside surface, was approximately $50.8 \mathrm{~mm}$ ( 2 inches), thereby dictating the length of each graphite sample. The diameter of each test sample was $25.4 \mathrm{~mm}$ (1 inch). All seventeen samples used in the graphite injection tests, outlined in Sections 4.3.2 and 5.3 were machined from PGX blocks.

The samples were clamped between two $25.4 \mathrm{~mm}$ diameter stainless steel vacuum fittings. The unobstructed cross-section of the fittings ensured the aerosol had maximum penetration potential. The two fittings and graphite sample were sealed with a heat activated adhesive lined shrink wrap. This process is referred to as potting. See Appendix D for photographs of potted graphite. A potted graphite schematic is shown in Figure 4.

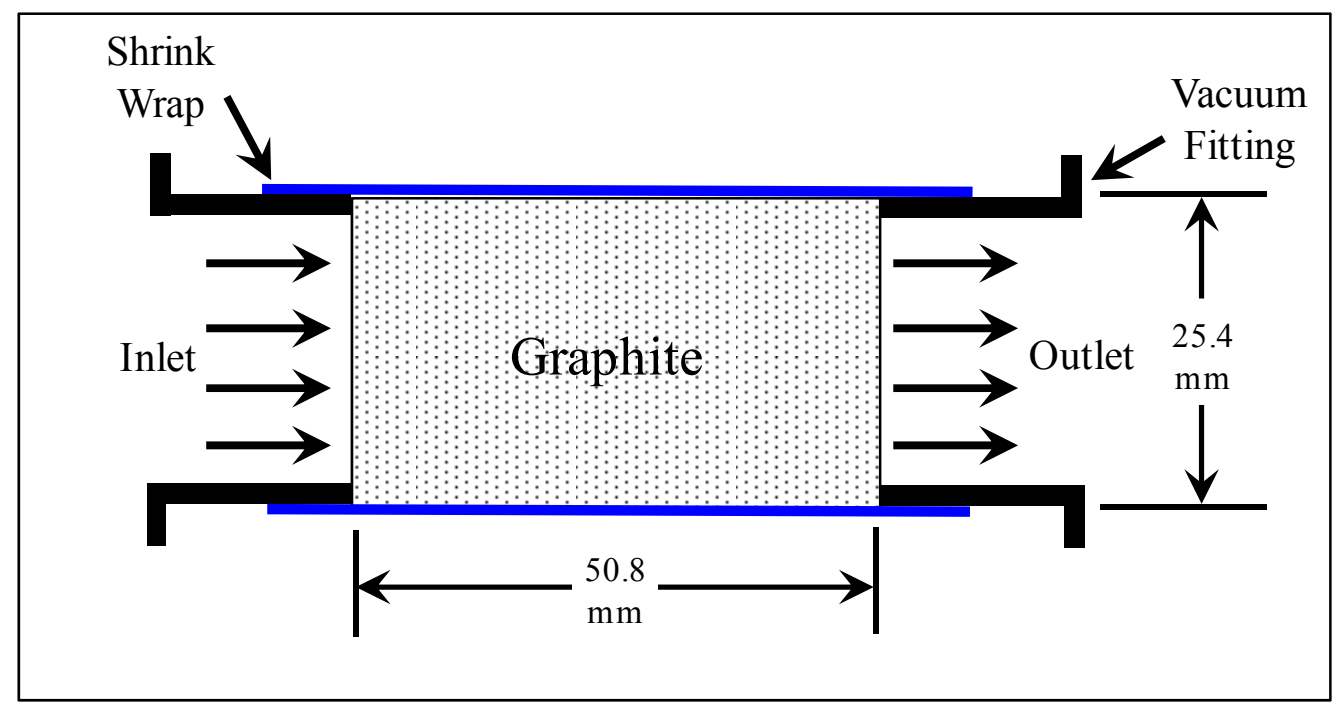

Figure 4: Potted graphite schematic.

Prior to subjecting the samples to aerosol flow, the graphite samples were characterized to understand expected flow rates, porosity, pore structure, uranium backgrounds and morphology. 


\subsection{Graphite Characterization Results}

The measured permeability (see Appendix A for a review of porosity and permeability) of each sample was plotted against the corresponding Darcy and compressible Darcy regressions, see Equations A.2 and A.3, respectively. Figure 5 shows an example of this analysis for PGX sample 19. The permeability for all measured samples was best determined from the compressible Darcy regression. While the regression difference appeared to be minimal, the resulting permeability variance was significant. For example, the Darcy and compressible permeability for PGX 19 were 259 and $201 \mathrm{md}$, respectively.

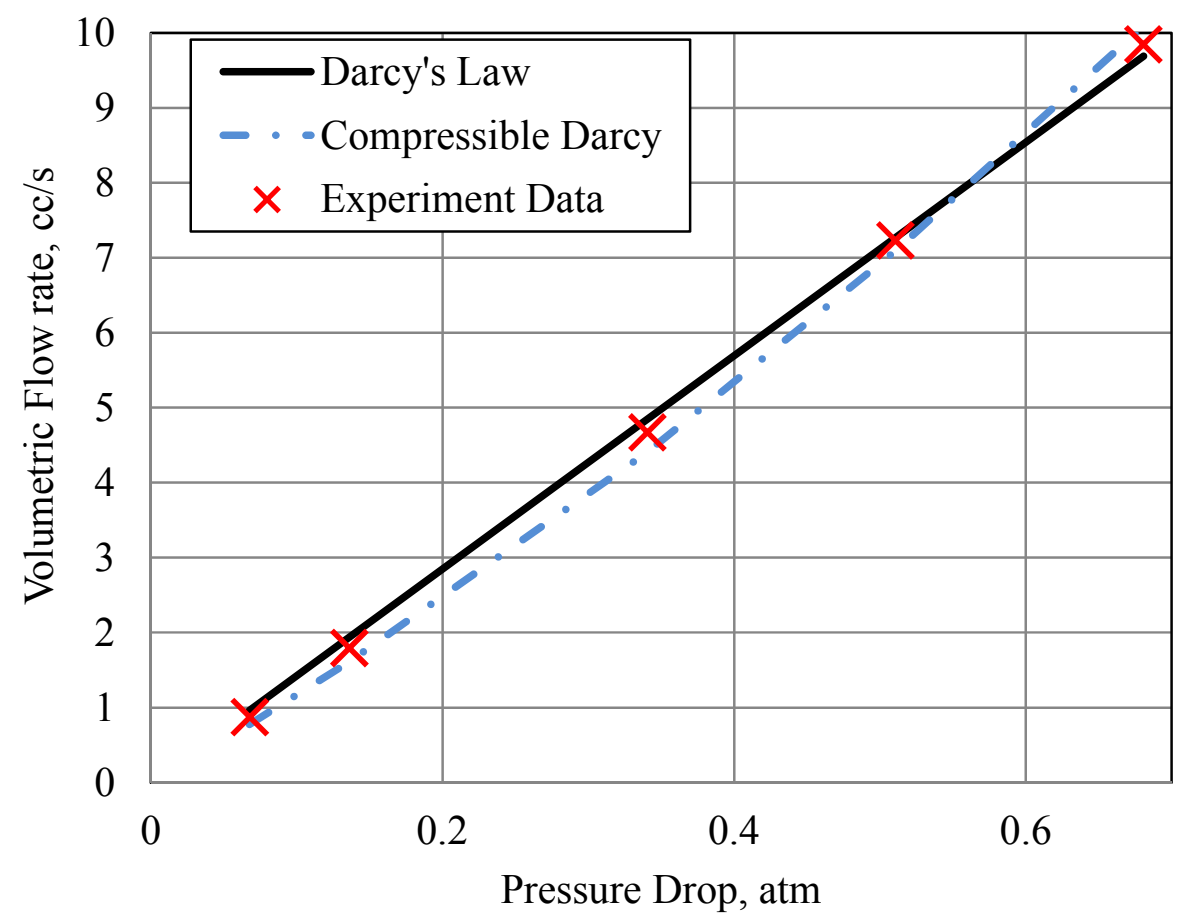

Figure 5: Permeability regressions for PGX graphite sample 19.

A summary of compressible Darcy permeability and porosity for all samples measured is given in Figure 6. The calculated volumetric flow rate for each sample is also shown. The average flow rate is 497 $\mathrm{cc} / \mathrm{min}$, which results in a face (or pore) velocity of $7.3 \mathrm{~cm} / \mathrm{s}$, assuming a porosity of $22.5 \%$. 
PNNL-21014

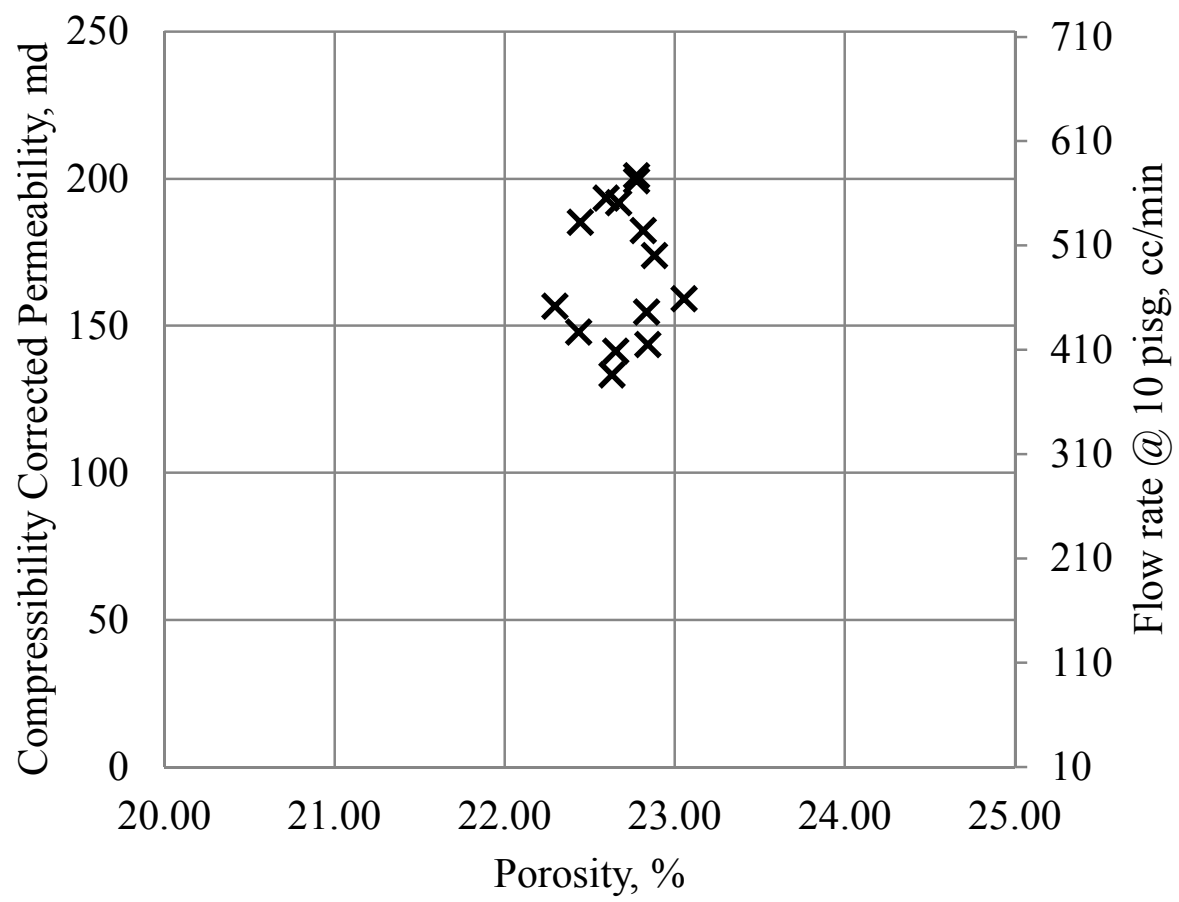

Figure 6. Graphite characterization results for 17 PGX graphite samples.

Mercury porosimetry analysis was also conducted on four graphite samples (roughly $1 \mathrm{~cm}^{3}$ ) to better understand the pore size distribution of various graphite types; PGX, RM, CX and BR. This analysis was supported by the Pixie Dust project, which is interested in multiple graphite types. However, for the present work, only PGX will be used and the other graphite types are shown for comparison only. The measurements were conducted by Quantachrome Instruments Inc. Mercury porosimetry consists of injecting a porous media with mercury and measuring the volume of mercury injected with increasing pressure. The pore size distribution is determined from the Washburn Equation (Le'on y Le'on, 1998), which relates the pore radius to the injection pressure.

The mercury porosimetry results are shown in Figure 7, where the vertical axis shows the slope of the intruded volume per log pore diameter which provides a distribution of pore throat diameters. The dominant pore size for the larger pores in the graphite samples is between 5 and 10 microns and there is also a significant amount of microporosity. It is evident that PGX graphite has pores extending from 5 20 microns, with many pores above 10 microns. 
PNNL-21014

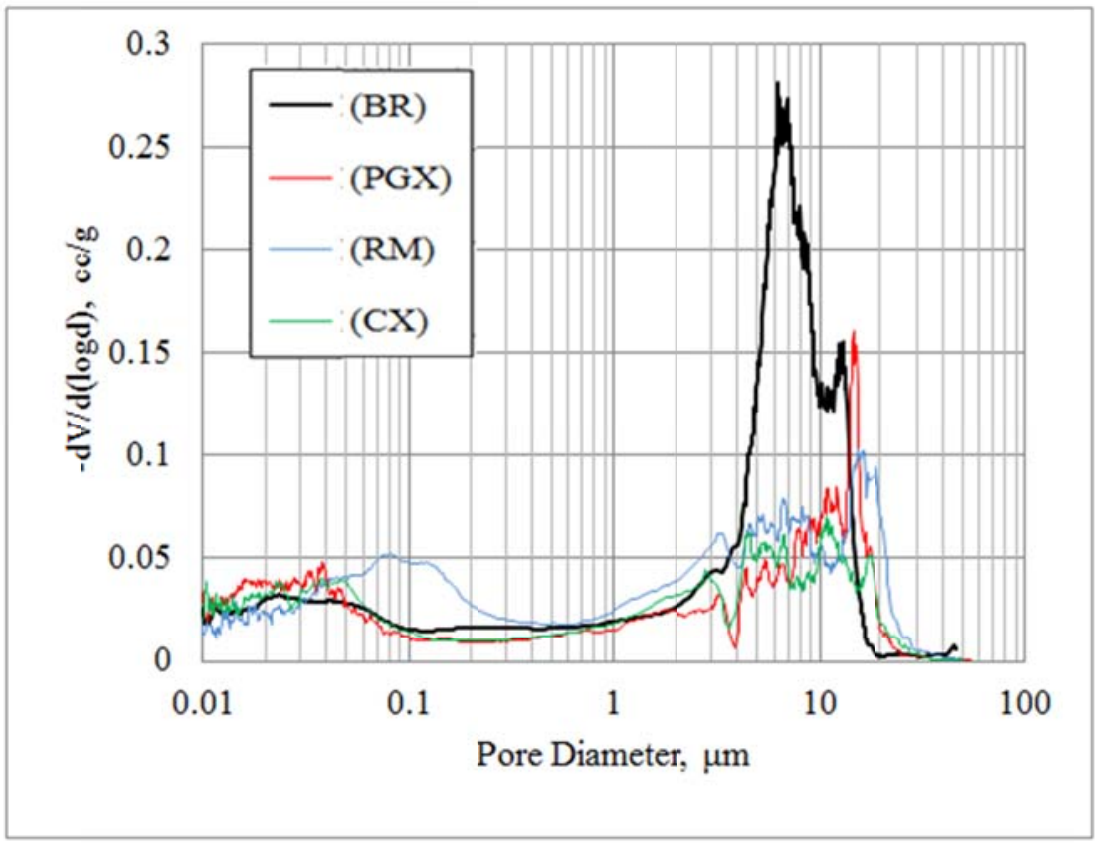

Figure 7: Mercury porosimetry pore size distribution results for BR, CX, PGX and RM graphite, where the vertical axis shows the slope of the intruded volume per log pore diameter which provides a measure of the pore volume.

The cumulative pore size distribution for the same graphite samples is shown in Figure 8 . The volume intruded has been normalized by the volume of each graphite sample; therefore, the last data point indicates the total porosity of the sample. Note, mercury porosimetry is only capable of measuring the connected pores, including dead-end pores, however, isolated pores are not measured.

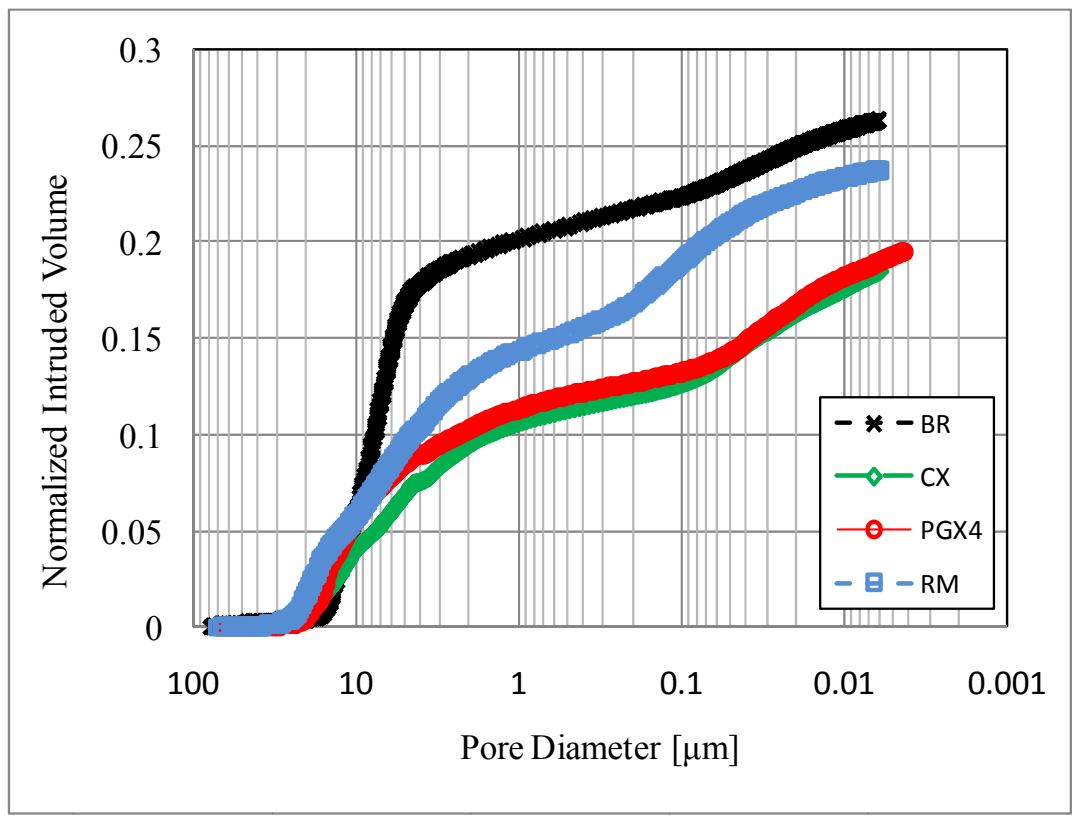

Figure 8: Mercury porosimetry cumulative pore size distribution results for BR, CX, PGX and RM graphite. 
PNNL-21014

\subsection{Graphite Uranium Background Content}

Three separate graphite samples (i.e., $50.8 \mathrm{~mm}$ cores) were analyzed to determine the background isotopic uranium content using the analytical technique discussed in Section 5.2. The averaged results are presented in Table 1, where the dominant uranium isotope in graphite is $U_{238}$. This is expected, as $U_{238}$ is a naturally occurring isotope.

Table 1: Average Background Uranium content in three PGX graphite samples.

\begin{tabular}{|c|c|}
\hline Isotope & $\begin{array}{c}\text { Concentration } \\
(\mathrm{ppb})\end{array}$ \\
\hline $\mathrm{U}_{234}$ & 0.00234 \\
\hline $\mathrm{U}_{235}$ & 0.0897 \\
\hline $\mathrm{U}_{238}$ & 19.13 \\
\hline $\mathrm{U}_{236}$ & Not Detected \\
\hline
\end{tabular}

\subsection{Graphite SEM Images}

Numerous SEM images of PGX graphite sample 17 were recorded using a JEOL JSM-840 instrument to provide insight regarding the pore size, topography and surface irregularities of the graphite.

Refer to Appendix A for images. Pores bodies ranging from $50-100 \mu \mathrm{m}$ can be seen in Figure 33, with large pore openings shown in Figure 34. This may appear to contradict the data shown in Figure 7;

however, mercury porosimetry does not measure the pore bodies (which can be quite large, as shown) but rather the pore throats, which provide the flow restriction. 
PNNL-21014

\subsection{Filtration Modeling}

\subsection{Model Overview}

Filtration of aerosol particles from a gaseous stream moving through a porous graphite sample was modeled using classical unit collector theory. In filtration, suspended particles in the fluid are removed when they adhere to the solid structure of the filter medium. If particles had infinitesimal size and followed the fluid streamlines perfectly, no contact would be made with the walls of the filter medium pores, and none of the particles would be captured. Several physical mechanisms bring finite sized particles into contact with the solid structure of the filter medium. Which mechanisms dominate in a given system depends upon particle size, the porous filter material geometry, and a variety of other factors. Usually one or two mechanisms dictate capture of a given particle size.

The important capture mechanisms include the following. See Figure 9 for a graphical representation.

- Diffusion: Diffusion moves particles between streamlines via the random Brownian motions arising from collisions with individual gas molecules. Diffusion capture often dominates for the smallest aerosol particles.

- Interception: Interception refers to the capture of particles which follow fluid streamlines almost perfectly, but are brought into contact with solid surfaces because of the finite size of the particles. Therefore, capture by interception occurs when a particle's center of mass follows a streamline which passes within one particle radius of a solid surface. Interception tends to be important for particles of intermediate size.

- Inertia: A particle's inertia may cause it to move between fluid streamlines as they change direction moving through a tortuous network of pores. This mechanism becomes important for heavy particles moving at high speeds.

- Gravity: Gravity forces cause heavy particles to drift downward within the flow-field. This mechanism is often called "settling" in aerosol systems or "sedimentation" in liquid systems and becomes important for heavy particles in regions of slower flow.

Other mechanisms, such as thermophoresis and electrostatic attraction can also dominate in aerosol systems where the associated forces are present.

A unit collector model employs a simple shape to represent elementary obstructions comprising the porous filter medium. Cylindrical unit collectors are often used to represent fiber filters, while spherical unit collectors are often used to represent granular beds. The flow field around the unit collector is usually assumed to be adequately approximated by some known analytical solution. This allows estimates to be made for capture rates of particles moving with the fluid flowing around the collector. Capture rates are estimated for fundamental capture mechanisms individually using simplifying assumptions, and then the capture rates are summed to estimate the total efficiency. 
PNNL-21014

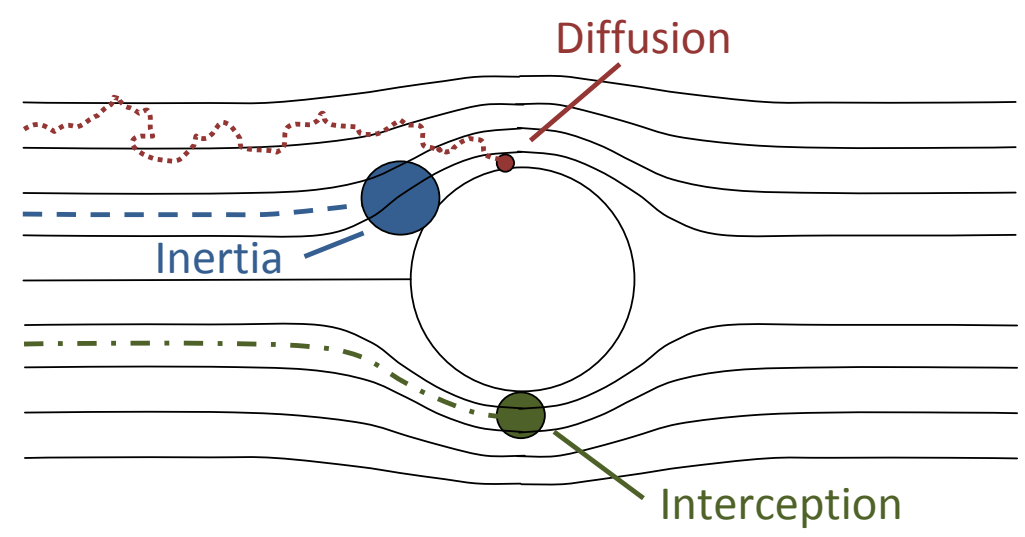

Figure 9: Several mechanisms leading to particle capture on a spherical unit collector.

Filtration by the porous graphite in this study was estimated using a spherical unit collector model, effectively approximating the graphite as a randomly packed bed of uniform spheres. Unit efficiency models for interception, diffusion, and gravity settling were taken from Elimelech et al. (1995), while the efficiency model for inertial impaction was taken from Otani et al. (1989). This model assumes spherical aerosol particles and no long range particle-medium interactions. The collision capture probability is assumed to be unity, therefore, any particle touching a solid surface is perfectly captured. Also, the model assumes the filtration properties (i.e., pore size, pore connectively, void fraction, etc.) are all homogeneous. For additional information regarding the unit collector model, see Appendix G.

Spherical unit collector models have been used successfully to model filtration in a variety of loose granular materials. They have also been applied to other porous filter materials, but opinions of their usefulness in this context vary in the literature (Konstandopoulos et al., 2002; Miyairi et al., 2006; Heikkinen and Harley, 2000). In general, a unit collector model can be expected to give the best results in a medium whose pore morphology closely resembles that used to derive the model and/or tune the model parameters.

\subsection{Conservative Aerosol Particle Size Estimation}

To facilitate planning of aerosol transport experiments, a unit collector model was employed to estimate the conservative or worst-case aerosol particle size. The worst-case particle size is defined as the size that would most likely penetrate furthest into the graphite. This required an estimate of a representative unit collector size for the graphite material. In a bed of spheres, the sphere diameter can be related to a representative pore size or to the bed permeability. If estimates for porosity, permeability, and representative pore size are taken to be $22.7 \%, 200 \mathrm{mD}$, and $10 \mu \mathrm{m}$, respectively, a collector diameter of $45 \mu \mathrm{m}$ might be considered reasonable to provide a rough estimate of the filter behavior. Other parametric values used in the model are shown in Table 2. The resulting estimated penetration depth as a function of particle size is shown in Figure 10. The model indicates that all particles larger than $0.6 \mu \mathrm{m}$ and smaller than $0.03 \mu \mathrm{m}$ would be captured very near the surface of the graphite material. However, between the aforementioned size ranges, the depth of penetration varies with particle size. As shown, the most penetrating particle size is predicted to be approximately $250 \mu \mathrm{m}$. Figure 10 shows that the concentration of each particle size will decay exponentially as a function of depth, indicating that $90 \%$ of the $250 \mu \mathrm{m}$ 
particles will be captured in slightly over $1.5 \mathrm{~mm}$ of the graphite material. It takes twice the distance to capture an additional $9 \%$ of the $250 \mu \mathrm{m}$ particles in the inlet stream.

Table 2: Unit Collector Model Parameters

\begin{tabular}{|c|c|}
\hline Model parameter & Value \\
\hline Gas temperature & $20{ }^{\circ} \mathrm{C}$ \\
\hline Gas molecular weight & 29 \\
\hline Gas pressure & $14.7 \mathrm{psig}$ \\
\hline Gas viscosity & $1.85 \mathrm{E}-5 \mathrm{~N} \mathrm{~s} / \mathrm{m}^{2}$ \\
\hline Gas superficial velocity & $3.75 \mathrm{~cm} / \mathrm{s}$ \\
\hline Medium collector diameter & $45 \mu \mathrm{m}$ \\
\hline Medium porosity & $22.7 \%$ \\
\hline Aerosol particle density & $10,970 \mathrm{~kg} / \mathrm{m}^{3}$ \\
\hline
\end{tabular}

Figure 11 shows the contributions of the various capture mechanisms to the predicted overall filtration efficiency for $\mathrm{UO}_{2}$ particles. Note that the diffusion mechanism dominates for very small particles, and the inertial mechanism dominates for larger particles. The maximally penetrating particle size occurs in the range where neither mechanism is dominant. The inertial mechanism is important because of the high density of the $\mathrm{UO}_{2}$ particles. Under the same flow and fluid conditions, interception would be the dominant mechanism for large particles with a specific gravity closer to 1.0, shifting the maximally penetrating particle size to a larger value.

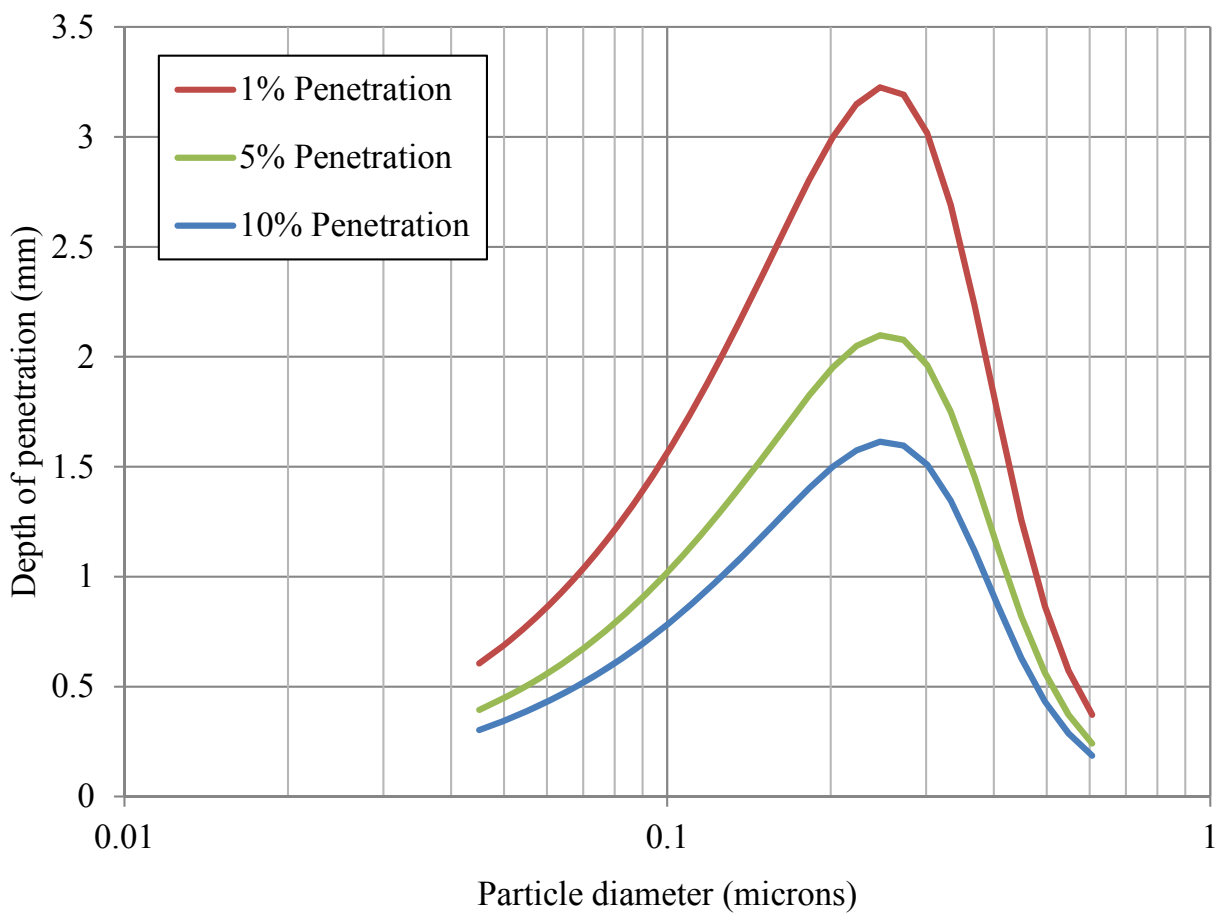

Figure 10: Estimation penetration depth as a function of particle size using unit collector model, assuming a face velocity of $3.75 \mathrm{~cm} / \mathrm{s}$. 
PNNL-21014

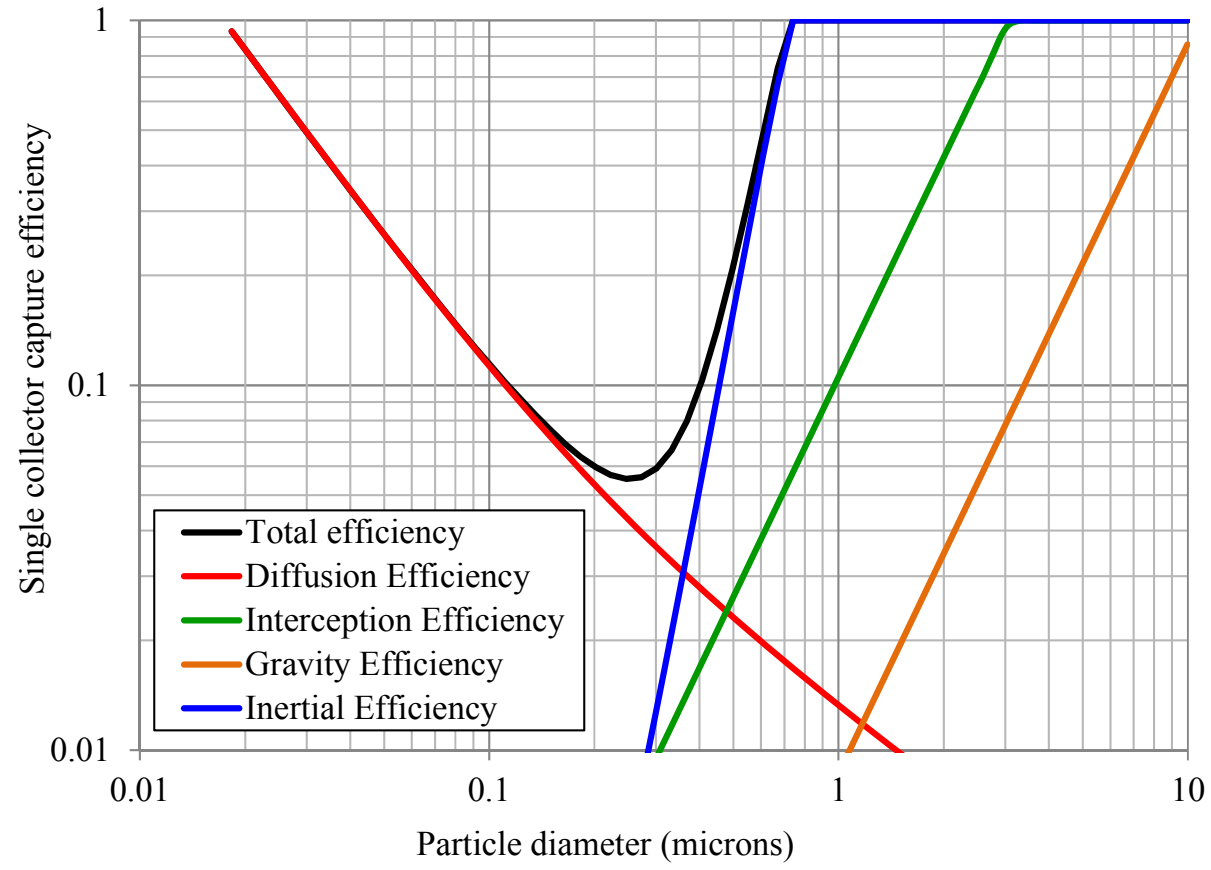

Figure 11: Contributions of various capture mechanisms to total capture efficiency for $\mathrm{UO}_{2}$ particles. 


\subsection{Aerosol Transport System}

\subsection{System Overview}

A pressure-driven aerosol flow system, termed the Aerosol Transport System (ATS), was designed to deliver an aerosol stream into the aforementioned graphite samples to determine the penetration extent of $\mathrm{UO}_{2}$ particles. Prior to injecting $\mathrm{UO}_{2}$ in a radiological environment, two surrogate materials; iron oxide $\left(\mathrm{Fe}_{2} \mathrm{O}_{3}\right)$ and tungsten carbide (WC), were evaluated to determine the best ATS design for $\mathrm{UO}_{2}$ testing. The following sections summarize the aerosol materials evaluated, the various ATS configurations and preliminary experimental results.

\subsection{Aerosol Material Characterization}

\subsubsection{Nature of Uranium Oxide Produced by $\mathrm{CO}_{2}$ Oxidation}

Based on Pearce, Whittle, and Hilton's (1969) review of the literature on metallic uranium oxidation by $\mathrm{CO}_{2}$, uranium dioxide $\left(\mathrm{UO}_{2}\right)$ is the only uranium oxide formed principally by

$$
\mathrm{U}+2 \mathrm{CO}_{2} \stackrel{\Delta}{\rightarrow} \mathrm{UO}_{2}+2 \mathrm{CO}
$$

The reaction product oxide contains varying amounts of carbon formed either by reaction of the uranium metal with the by-product $\mathrm{CO}$ via

$$
U+C O \stackrel{\Delta}{\rightarrow} U_{2}+2 C
$$

or by thermal decomposition of the CO.

$$
2 \mathrm{CO} \underset{\leftarrow}{\longrightarrow} \mathrm{CO}_{2}+\mathrm{C}
$$

The carbon content depends on temperature. Below $670^{\circ} \mathrm{C}$, the carbon content reaches equilibrium where deposited carbon reacts with $\mathrm{CO}_{2}$ to reform $\mathrm{CO}$ that diffuses into cracks formed in the oxide. At higher temperatures the carbon content is linearly related with the extent of oxidation with formation of the uranium carbides $\mathrm{UC}$ and $\mathrm{UC}_{2}$ by reaction of uranium metal with $\mathrm{CO}$ via the reactions

$$
2 U+2 \mathrm{CO} \stackrel{\Delta}{\rightarrow} U \mathrm{O}_{2}+U C_{2}
$$

and

$$
3 U+2 \mathrm{CO} \stackrel{\Delta}{\rightarrow} U O_{2}+2 U C .
$$

Thus based on Pearce, Whittle, and Hilton (1969), the product of the reaction of uranium metal with $\mathrm{CO}_{2}$ will be $\mathrm{UO}_{2}$ containing deposited carbon or uranium carbides depending on the reaction temperature.

In contrast, Alire, Czanderna, and Wittaker (1977) found that higher oxides $\mathrm{UO}_{2+\mathrm{x}}$ formed in their studies of the reaction of uranium metal with $\mathrm{CO}_{2}$. Their studies used uranium powder and foil and they took the 
reactions to completion. Their review of past work found only studies that did not completely oxidize the uranium to its final stable state. Dominey, Morley, and Young (1966) found that $\mathrm{UO}_{2}$ exposed to $\mathrm{CO}_{2}$ in a sealed vessel, irradiated, and heated was oxidized to $\mathrm{UO}_{2}+x$. Alire, Czanderna, and Wittaker (1977) provide the following reaction

$$
\mathrm{U}+(2+x) \mathrm{CO}_{2} \stackrel{\Delta}{\rightarrow} \mathrm{UO}_{2+x}+(2+x) \mathrm{CO}
$$

to explain their results. Their x-ray diffraction (XRD) spectroscopic analysis found no indication of any uranium carbide formation nor did the gas pressure decrease due to conversion of $\mathrm{CO}_{2}$ to carbon.

It was beyond the scope of this testing to determine the form of $U$ present in graphite reactors. This study assumed that the low operational temperatures of a graphite reactor was likely to produce $\mathrm{UO}_{2}$ determined in the Pearce, Whittle, and Hilton's (1969) review rather than the $\mathrm{UO}_{2}+\mathrm{x}$ equilibrium form that appeared to require more stringent conditions.

\subsubsection{Carbon Dioxide Oxidation of Uranium}

In order to understand the expected PSD of reactor-oxidized $\mathrm{UO}_{2}$ particles and compare to conservative model estimates, an experiment was proposed to oxidize uranium metal in an environment prototypic of a graphite reactor. The resulting $\mathrm{UO}_{2}$ material would be characterized and used in the ATS system.

Thermogravimetric analysis (TGA) was employed to characterize the thermal reaction of uranium metal with $\mathrm{CO}_{2}$ to identify a temperature that would produce material at a reasonable rate given the very slow reaction kinetics (Pearce et al. 1969). This proved to be a difficult to implement strategy since the $\mathrm{CO}_{2}-$ oxidation rate is much slower than oxidation by oxygen and the experiment experienced air ingress that was difficult to mitigate. As a result, characteristic uranium oxide could not be produced for graphite injection testing.

A discussion of the attempts to produce $\mathrm{UO}_{2}$ through carbon dioxide oxidation and subsequent testing are presented in Appendix F.

\subsubsection{Uranium Oxide Isotopic Composition}

Due to the difficulty associated with oxidizing $\mathrm{U}$ with $\mathrm{CO}_{2}$ to obtain $\mathrm{UO}_{2}$, a substitute $\mathrm{UO}_{2}$ material, identified as Redding Enriched Uranium (EU), was obtained. Isotopic analysis was performed and the material was found to have isotopes 234, 235, 236 and 238 present. The concentration data is presented in Table 3. The $\mathrm{U}_{236}$ isotope is characteristic of irradiated uranium and, therefore, will not be present in the graphite, as confirmed in Section 2.3. Therefore, the EU was used for all $\mathrm{UO}_{2}$ aerosol testing and if the $\mathrm{U}_{236}$ isotope was discovered in the graphite, it could only be from the EU material, providing a defensible analysis approach.

Table 3: Redding EU isotopic composition data.

\begin{tabular}{|c|c|}
\hline Isotope & Concentration \\
\hline $\mathrm{U}_{234}$ & $260 \mathrm{ppm}$ \\
\hline $\mathrm{U}_{235}$ & $4.3 \mathrm{wt} \%$ \\
\hline
\end{tabular}




\begin{tabular}{|c|c|}
\hline $\mathrm{U}_{236}$ & $250 \mathrm{ppm}$ \\
\hline $\mathrm{U}_{238}$ & $95.65 \mathrm{wt}^{\mathrm{o}} \%$ \\
\hline
\end{tabular}

The as-received EU was examined with optical microscopy and found to contained a broad particle size distribution. Prior to using the EU in aerosol tests, the material was sieved to remove large particles and ground using a mill to ensure that some particles in the 1 to $0.1 \mu \mathrm{m}$ range were present since particles in this size range were predicted by modeling to be most likely to penetrate furthest into the graphite. SEM images were taken to evaluate the resulting ground materials. An example SEM image is shown in Figure 12 , where many submicron particles can be seen. However, large fundamental particles and agglomerates are also present. Additional SEM images are provided in Appendix D.

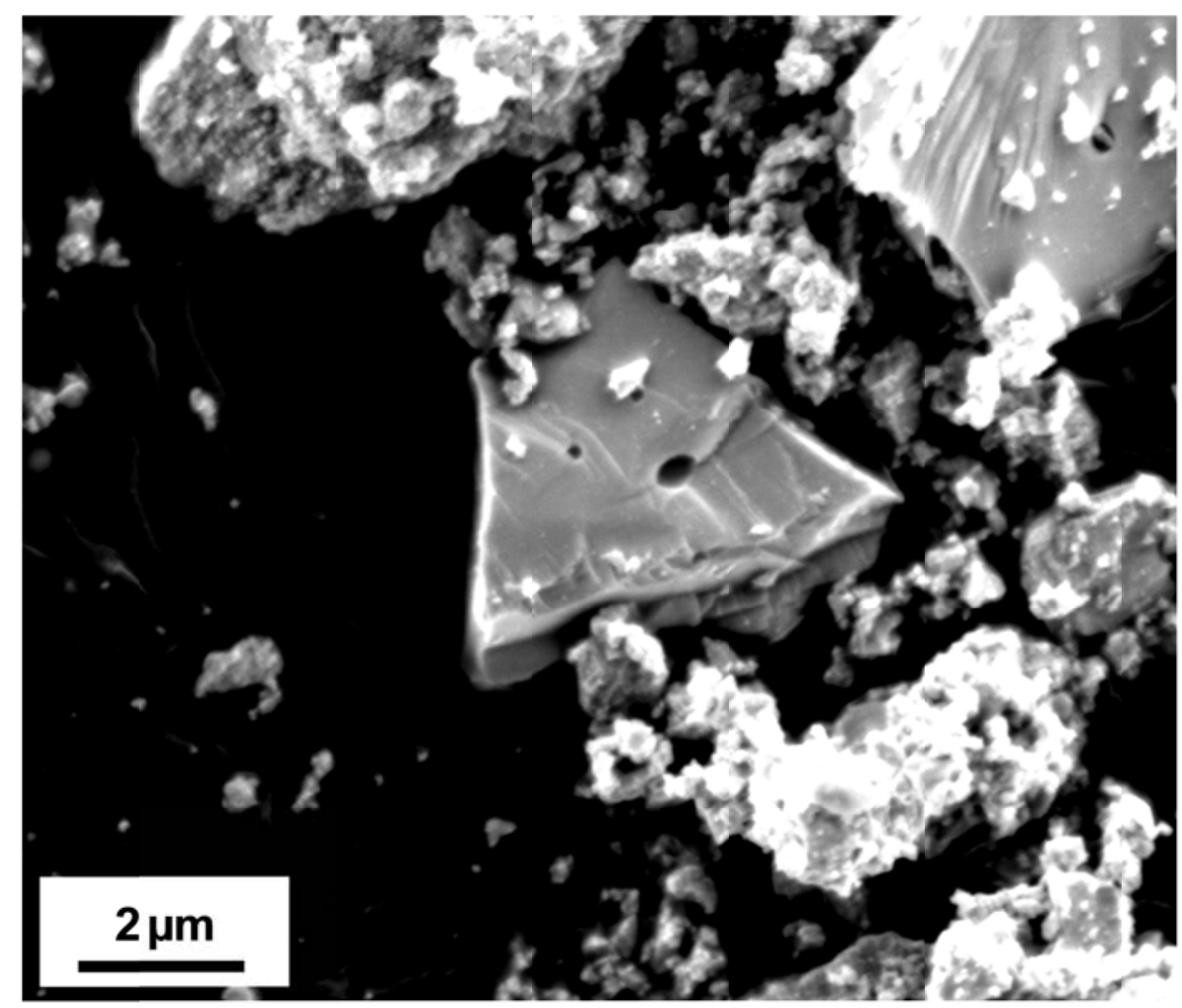

Figure 12: High magnification SEM image of uranium oxide. Both fundamental particles and agglomerates exist for a broad size range.

\subsubsection{Surrogate Materials}

Two surrogate materials were selected to test the ATS (see Section 4.3) prior to using the $\mathrm{EU} \mathrm{UO}_{2}$, due to the complexity of working in a radiological environment. Iron oxide and tungsten carbide were chosen as surrogate materials to provide a range of particle densities that bounded the $\mathrm{UO}_{2}$ particle density. Particle density will be a dominant property effecting aerosol transport, especially at the higher densities of WC and $\mathrm{UO}_{2}$. Additionally, as shown in

Figure 13 and Figure 14, the particle size distributions of the surrogate materials contain a reasonable quantity of particles of the size fraction in the model-predicted conservative range discussed in Section 
3.2. The density and size range of the aerosol materials evaluated are summarized in Table 4. SEM images of iron oxide and tungsten carbide are also provided in Appendix D; Figure 43 and Figure 44, respectively.

Table 4: Characteristics of aerosol materials tested using ATS.

\begin{tabular}{|c|c|c|}
\hline Material & $\begin{array}{c}\text { Density } \\
(\mathrm{g} / \mathrm{cc})\end{array}$ & $\begin{array}{c}\text { Particle Size Range } \\
(\mu \mathrm{m})\end{array}$ \\
\hline Iron Oxide $\left(\mathrm{Fe}_{2} \mathrm{O}_{3}\right)$ & 5.24 & $0.2-30^{(\mathrm{a})}$ \\
\hline Tungsten Carbide $(\mathrm{WC})$ & 15.8 & $0.4-5^{(\mathrm{a})}$ \\
\hline Uranium Oxide $\left(\mathrm{UO}_{2}\right)$ & 10.97 & $0.1-5^{(\mathrm{b})}$ \\
\hline
\end{tabular}

(a) Determined using a Malvern Mastersizer 2000.

(b) Rough estimate from SEM images

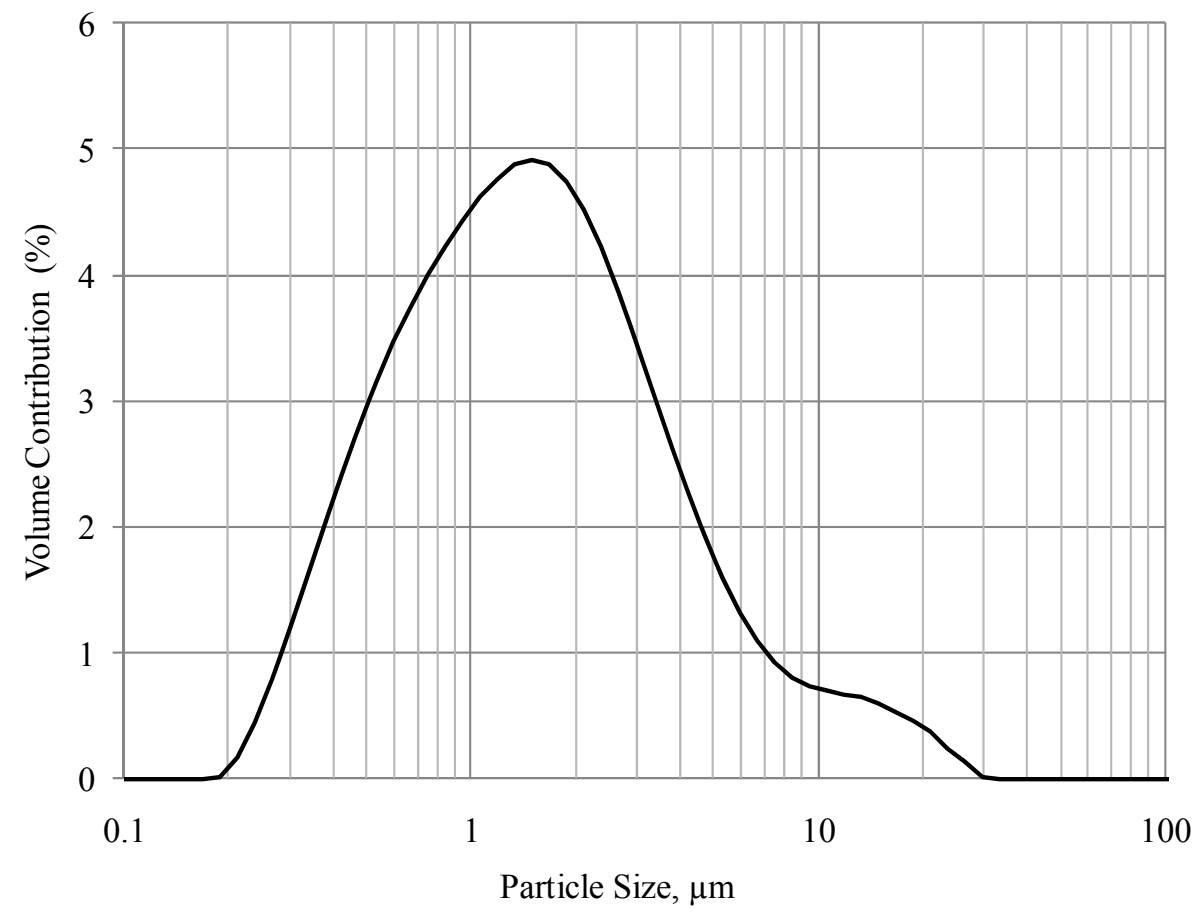

Figure 13: Iron oxide particle size distribution. 
PNNL-21014

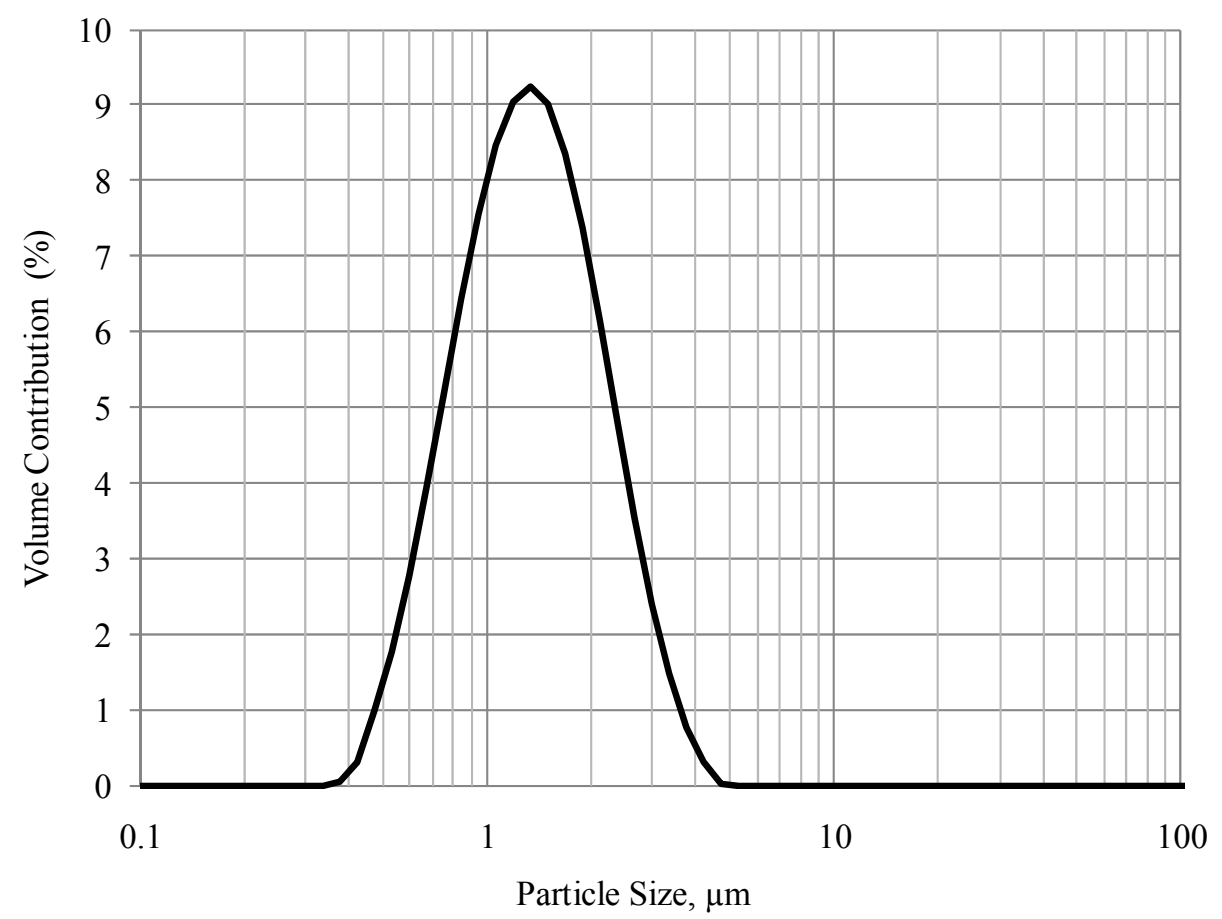

Figure 14: Tungsten carbide particle size distribution.

\subsection{Phase I: Surrogate Aerosol Experimental Apparatus}

The ATS was developed using an initial surrogate testing phase followed by a second radiological testing phase. This approach allowed the ATS system to be configured, tested and tuned using a surrogate material prior to developing the radiological system.

In phase I, two configurations were utilized; a 3-stage filter configuration and a graphite injection configuration. The 3-stage filter configuration was used to develop methods that ensured that the proper size distribution of particles was introduced to the graphite sample. This configuration also helped determine the approximate size and quantitative estimates of the mass of aerosol particles that would be introduced to the graphite sample. The second configuration allowed for particle penetration tests in graphite. The specifics of each configuration and testing results are described in subsequent sections.

\subsubsection{Filter Testing}

The ATS system in the three filter configuration is shown in Figure 15. In this configuration, the system was comprised of a regulated pressurized gas cylinder connected to a pressure gauge and an isolation valve; a particulate loading tee; a mixing chamber, and a series of filters. For each test, a known quantity of particulate material was placed into the loading tee; the isolation valve was opened, the resulting gas flow suspended a portion of the particulate material. The gas with suspended particles passed through a $762 \mu \mathrm{m}\left(1 / 16^{\text {th }}\right.$ inch) Swagelok reducer fitting at high speed to impart significant energy to break-up agglomerates and disperse the particles. The aerosol stream then entered a mixing chamber to develop a 
homogeneous dispersion of particles prior to entering the test section. The mixing chamber also provided space for large agglomerates to settle, rather than impact and clog the face of the material (i.e., filter or graphite) in the test section. During filter testing, the test section was comprised of three filters arranged in decreasing average pore sizes of 5, 2.5 and $0.1 \mu \mathrm{m}$. The filter specifications and photographs are shown in Appendix E. The flow rate through the filters was controlled by placing a spare potted graphite sample of known permeability downstream of the filter stage and adjusting the inlet pressure, which was maintained at $10 \mathrm{psig}$. The test section exhaust was vented into a hood to control any release of particulate material.

The filter sizes were chosen to trap particles in a step-wise approach. The $5 \mu \mathrm{m}$ filter captured any large agglomerates that might plug the finer filters. The 2.5 and $0.1 \mu \mathrm{m}$ filters allowed for a rough assessment of the size of particles that pass through the $5 \mu \mathrm{m}$ filter and helped ensure that particles in the desired particle size range of $0.1-1 \mu \mathrm{m}$ would be delivered to the surface of the graphite during testing. After each test, the filters were analyzed via digestion and inductively coupled plasma-optical emission spectrometry (ICP-OES) or ICP- mass spectrometry (ICP-MS) to determine the quantity of material collected on each filter. This technique was not precise due to the broad capture efficiency of each filter (i.e., a $5 \mu \mathrm{m}$ filter will capture some small particles) but allowed a rough quantification of the aerosol PSD that was expected to reach the graphite sample.

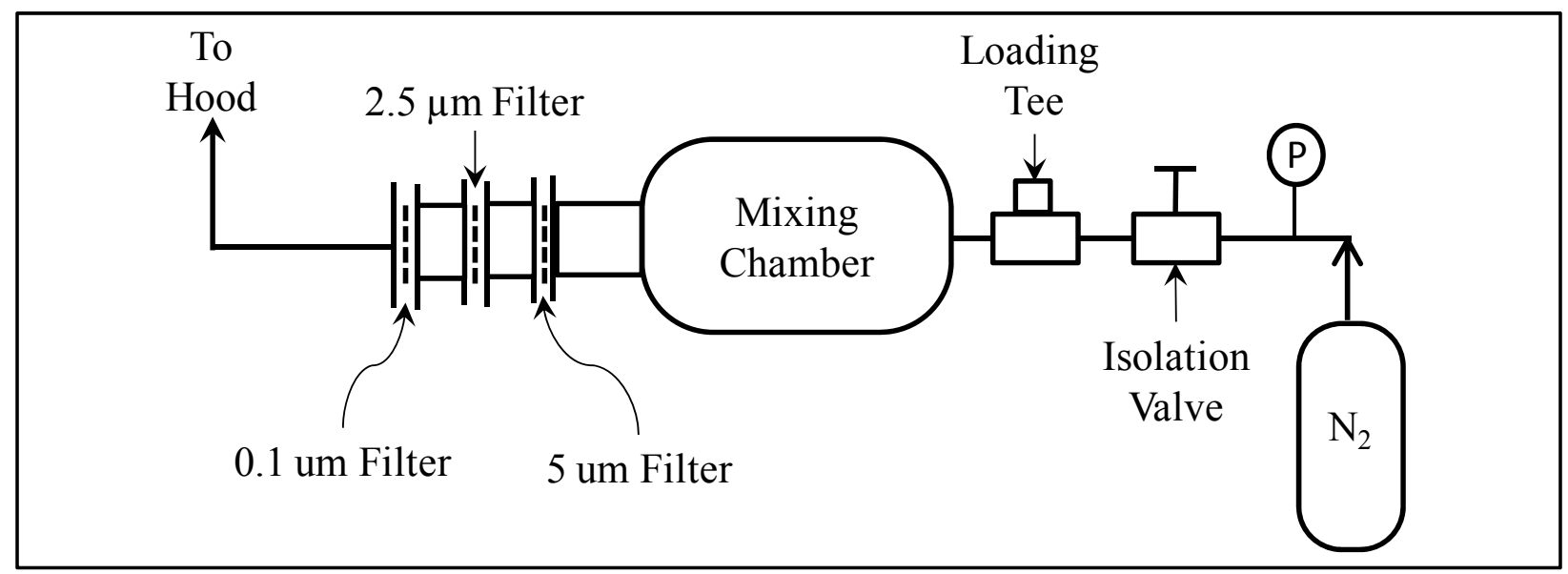

Figure 15: ATS 3-stage filter test configuration schematic.

Initial experiments were performed using nitrogen $\left(\mathrm{N}_{2}\right) . \mathrm{N}_{2}$ was a reasonable gas for scoping tests having a viscosity that was only $15 \%$ higher than $\mathrm{CO}_{2}$. However, the lower density of $\mathrm{N}_{2}$ (half that of $\mathrm{CO}_{2}$ ), lead to the use of $\mathrm{CO}_{2}$ for all $\mathrm{UO}_{2}$ aerosol testing to ensure consistency with prototypic reactor coolant gas. See Appendix E for gas properties.

\subsubsection{Graphite Injections}

The graphite injection setup, shown in Figure 16, was identical to the 3-stage filter test configuration with the exception of the test section (see Appendix E for photographs). The two smaller pore size filters were replaced with a graphite sample. Initial filter testing concluded that the $5 \mu \mathrm{m}$ filter was required to prefilter the aerosol stream prior to flowing through the graphite. The pre-filter prevented large particles from reaching the graphite face and clogging pores, which might artificially decrease transmission. A few 
qualitative graphite injection tests were performed with iron oxide surrogate material to ensure that an aerosol stream was introduced to the graphite sample.

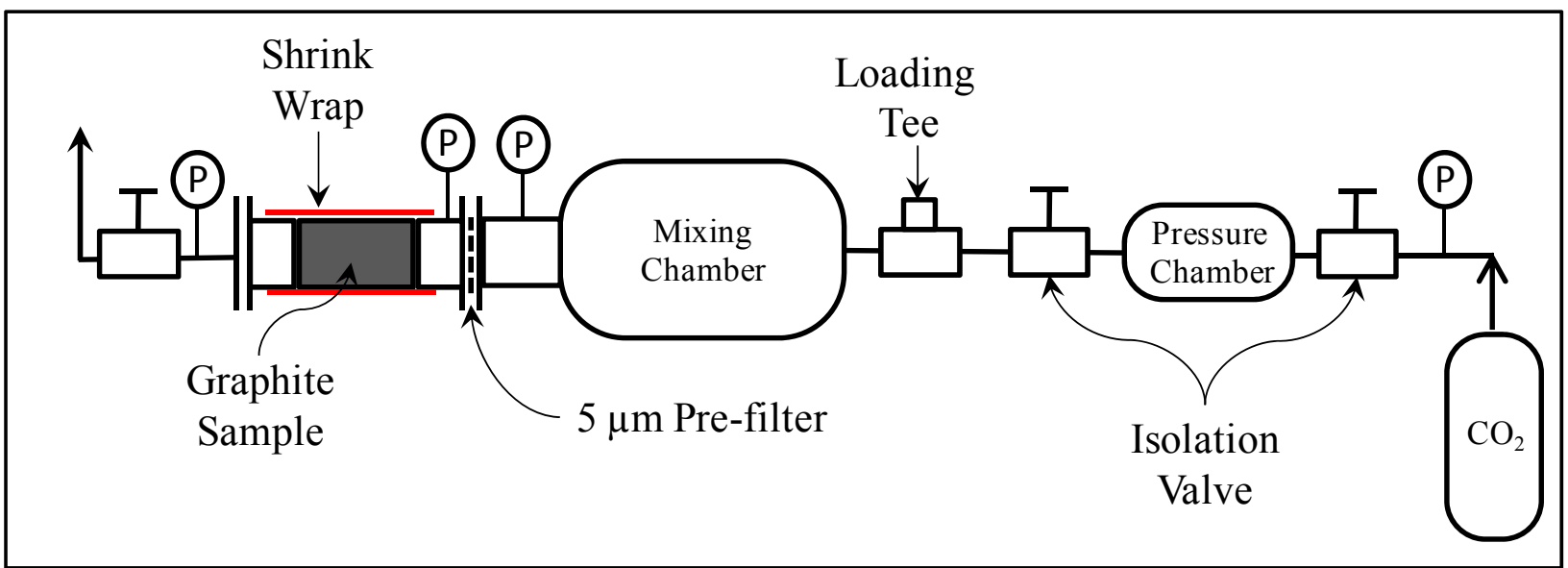

Figure 16: ATS graphite injection test configuration schematic.

\subsection{Phase II: Uranium Oxide Aerosol Experimental Apparatus}

Upon completing the surrogate material testing, a Phase II ATS system was designed for use in a radiological environment. The Phase I ATS system provided invaluable insight into the improvements and enhancements necessary for the Phase II system. The improved system is shown schematically in Figure 17, where 
PNNL-21014

Table 5 provides a hardware identification summary. Photos of the Phase II ATS are shown in Figure 53. Again, for filter testing the graphite sample was replaced by 2.5 and $0.1 \mu \mathrm{m}$ filters. Due to the cost and complexity of radiological experiments, the Phase II ATS system was enhanced to include user feedback features and additional safety controls, such as filter and graphite pressure drop measurement ( 
Table 5, ID 11 and 12), exit gas flow rate, bubbling pressure indicator for purging and additional isolation valves.

Due to the reactive nature of $\mathrm{UO}_{2}$ in the presence of oxygen, the Phase II ATS included capabilities that allowed the system to be purged with $\mathrm{CO}_{2}$ prior to each test. After loading the graphite sample, the entire system was purged with $\mathrm{CO}_{2}$. At the completion of $\mathrm{CO}_{2}$ purging, the majority of the system volume was isolated from ambient air via valve 2, 3, 4 and 5. The loading tee, containing a measured amount of $\mathrm{UO}_{2}$, was inserted between valve 2 and 3 . The pressure chamber was then charged with $\mathrm{CO}_{2}$ at the injection pressure which was generally higher than the flow pressure and isolated from the flow pressure via valve 1. The purge $\mathrm{CO}_{2}$ was then exhausted via valve 5 using a visual bubbling system to show when the release was complete and to prevent any backflow of ambient air. This step reduced pressure in the mixing chamber to prevent back-flow. The $\mathrm{UO}_{2}$ was then injected into the system by opening valves 3 and 4 to allow flow through the graphite, valve 2 to allow a high pressure, low volume shot of injection $\mathrm{CO}_{2}$ and then valve 1 to generate the sustained pressure necessary to drive the flow through the graphite. 
PNNL-21014

Table 5: Identification of the radioactive environment ATS components.

\begin{tabular}{|c|c|c|c|}
\hline ID Number & Description & ID Number & Description \\
\hline 1 & Regulated $\mathrm{CO}_{2}$ cylinder & 8 & Potted graphite sample \\
\hline 2 & Pressure gauge & 9 & Gas flow meter \\
\hline 3 & Pressure relief valve & 10 & Exhaust \\
\hline 4 & Check valve & 11 & Graphite pressure drop transmitter \\
\hline 5 & Isolation valve & 12 & Pre-filter pressure drop transmitter \\
\hline 6 & Loading tee & 13 & Bubbling pressure indicator \\
\hline 7 & $5 \mu \mathrm{m}$ pre-filter & 14 & Data acquisition system \\
\hline
\end{tabular}




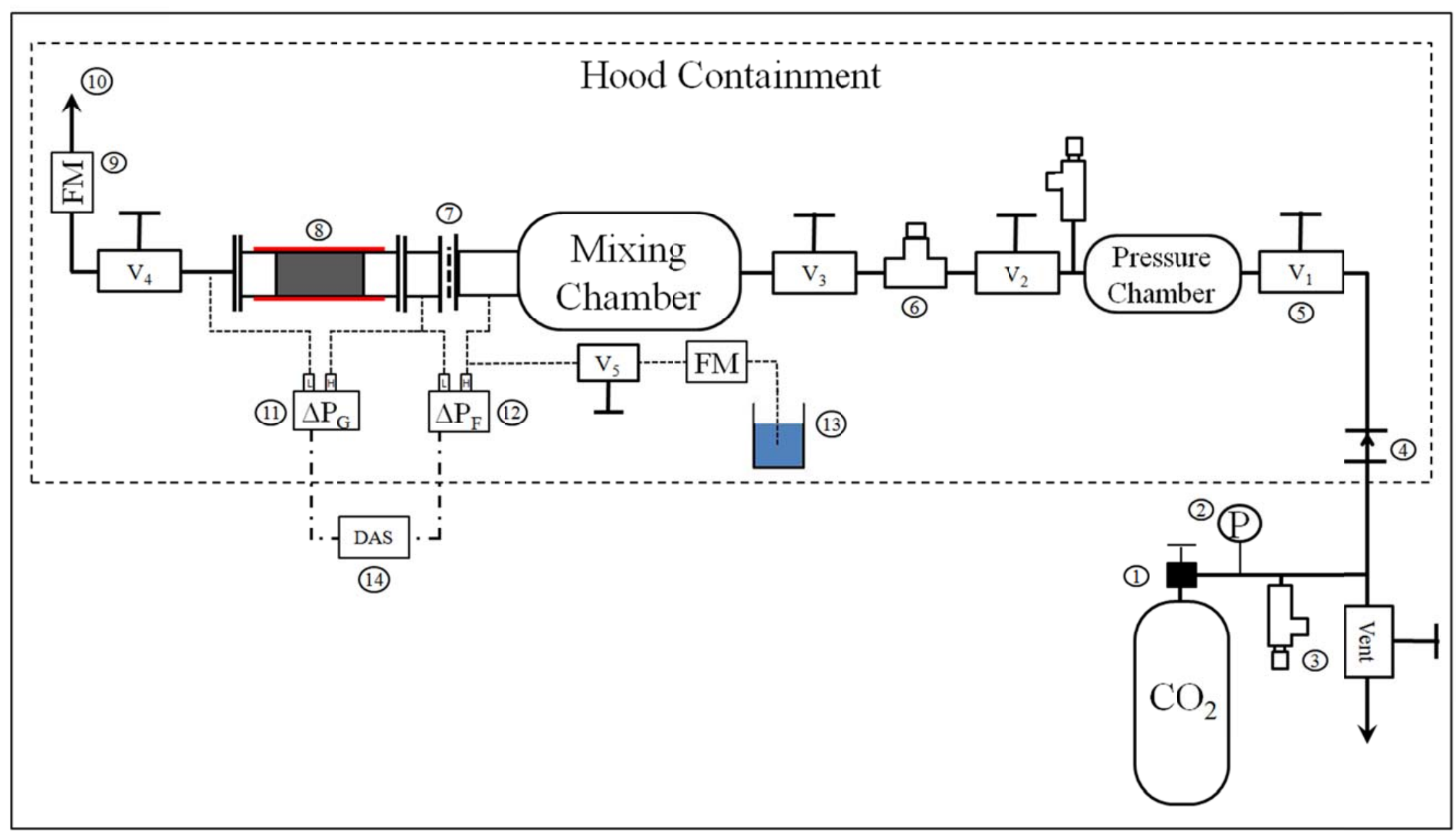

Figure 17: Radioactive environment ATS graphite injection test configuration schematic. 
PNNL-21014

\subsection{3-stage Filter Test Results}

\subsubsection{Iron Oxide}

An iron oxide aerosol stream was evaluated using the Phase I 3-stage testing configuration. A baseline loading mass of approximately $50 \mathrm{mg}$ was established based on available quantity of $\mathrm{UO}_{2}$ and number of desired tests. The loaded mass was defined as the amount of material placed into the loading tee.

Subsequently, the injected mass was the quantity of material removed from the loading tee by the flowing gas. The collected mass was the amount of material trapped on the three filters downstream of the loading tee. The injection pressure was varied from a minimum of $10 \mathrm{psi}$ based on the flow pressure to a high of 30 psi. Three filter tests were performed using $\mathrm{Fe}_{2} \mathrm{O}_{3}$ with the specifications shown in Table 6 where the injection pressure and mass loaded were varied to understand the impacts on injected mass, collection efficiency and PSD. The baseline run-time for all tests was 10 minutes, which provided adequate time for three mixing chamber volumes to pass through the filters.

Table 6: Iron Oxide filter test data.

\begin{tabular}{|c|c|c|c|c|}
\hline $\begin{array}{c}\text { Test } \\
\text { Number }\end{array}$ & $\begin{array}{c}\text { Injection } \\
\text { Pressure } \\
(\mathrm{psig})\end{array}$ & $\begin{array}{c}\text { Mass } \\
\text { Loaded } \\
(\mathrm{g})\end{array}$ & $\begin{array}{c}\text { Mass } \\
\text { Injected } \\
(\mathrm{g})\end{array}$ & $\begin{array}{c}\text { Injection } \\
\text { Efficiency } \\
(\%)\end{array}$ \\
\hline 2 & 10 & 0.043 & 0.007 & 16 \\
\hline 3 & 30 & 0.047 & 0.010 & 21 \\
\hline 4 & 30 & 0.012 & 0.006 & 50 \\
\hline
\end{tabular}

After testing, the filters (see an example filter in Figure 50 (d) in Appendix E) were chemically digested and analyzed using ICP-OES or ICP-MS ${ }^{1}$ to determine the mass collected on each filter.

The $\mathrm{Fe}_{2} \mathrm{O}_{3}$ filter test results are summarized in Figure 18 and Figure 19, where the mass and percent collected on each set of filters are shown, respectively. Losses in these figures are defined as material that was injected into the system but did not show up on any of the filters. The detection limit (DL) of the instrument is indicated in Figure 18 and is dependent on the acid digestion solution volume and the instrument sensitivity for a specific element. Any values below the DL should be regarded as immeasurable and, therefore, equivalent to zero.

As shown in Table 6 and Figure 19, there are significant losses (e.g., $50-90 \%$ ) associated with this system. This is expected due to the simplistic method of aerosol generation. The results of the $\mathrm{Fe}_{2} \mathrm{O}_{3}$ filter tests generated the following conclusions:

\footnotetext{
${ }^{1}$ Perkin Elmer Optima 3300-DV inductively coupled plasma-optical emission spectrophotometer (ICP-OES) or a Perkin Elmer Elan-DRC inductively coupled plasma-mass spectrometer (ICP-MS). Both instruments were calibrated using NIST-traceable single element standards following PNNL-approved operating procedures (PNNLAGG-415 Rev. 2 and PNNL-AGG-ICP-AES Rev. 2) under the Environmental Sciences Laboratory's quality assurance program (ESL-QAP Rev. 3). The ICP-OES and ICP-MS measured the mass of a particular element, either $\mathrm{Fe}$ or $\mathrm{W}$, and used the chemical composition of the particulate, either $\mathrm{Fe}_{2} \mathrm{O}_{3}$, or WC to calculate the mass collected on each filter. Blank filters were also analyzed to verify no background Fe or W were present in the filters.
} 
- Small particles within desired size range can be delivered to graphite samples

- The mass of small particles is measurable and can be quantified

o $\sim 90 \mu \mathrm{g}$ on the 2.5 and $0.1 \mu \mathrm{m}$ filters

- Increased injection pressure results in more smaller particles that penetrate the $5 \mu \mathrm{m}$ filter

o Minimum 30 psi injection pressure used for subsequent tests

- Decreasing mass loaded significantly reduces percent of small particles

o Minimum of $50 \mathrm{mg}$ used for subsequent tests

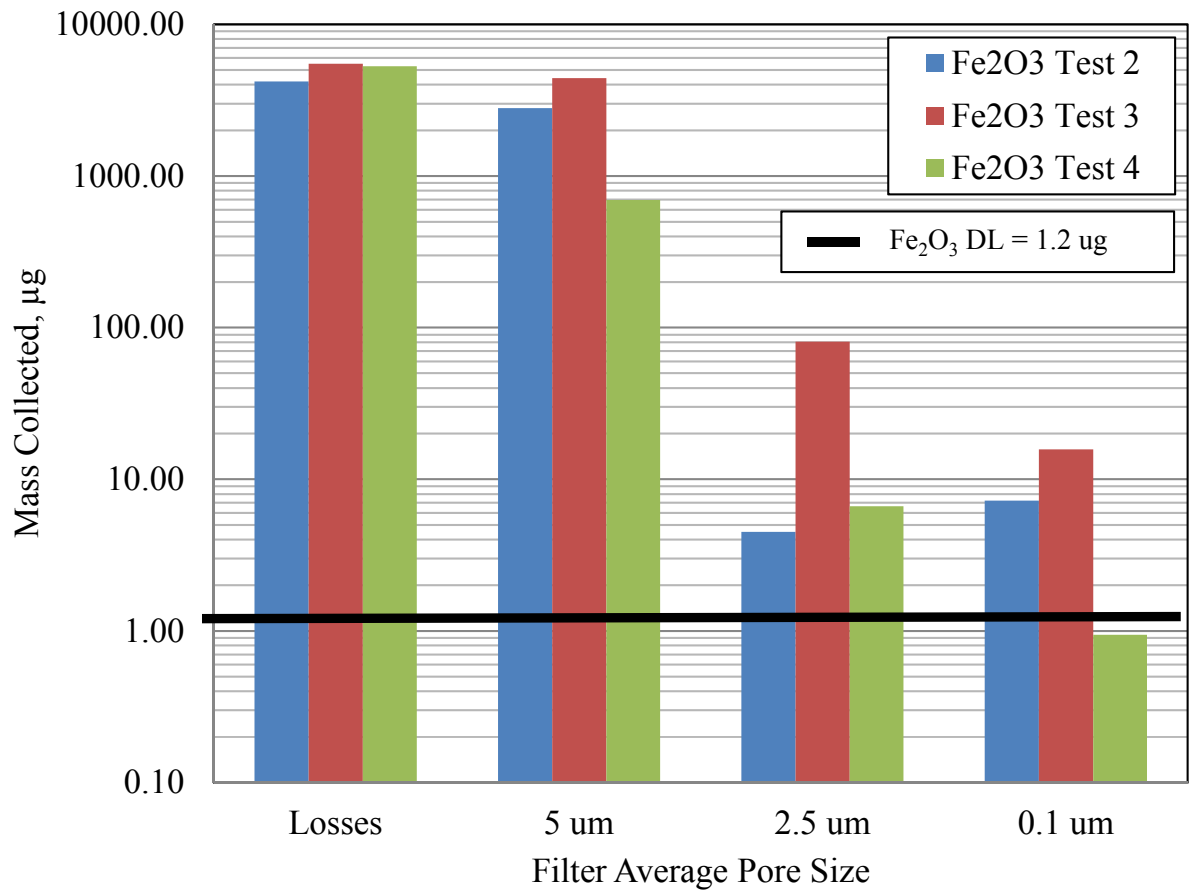

Figure 18: Iron oxide filter test mass collected results. 


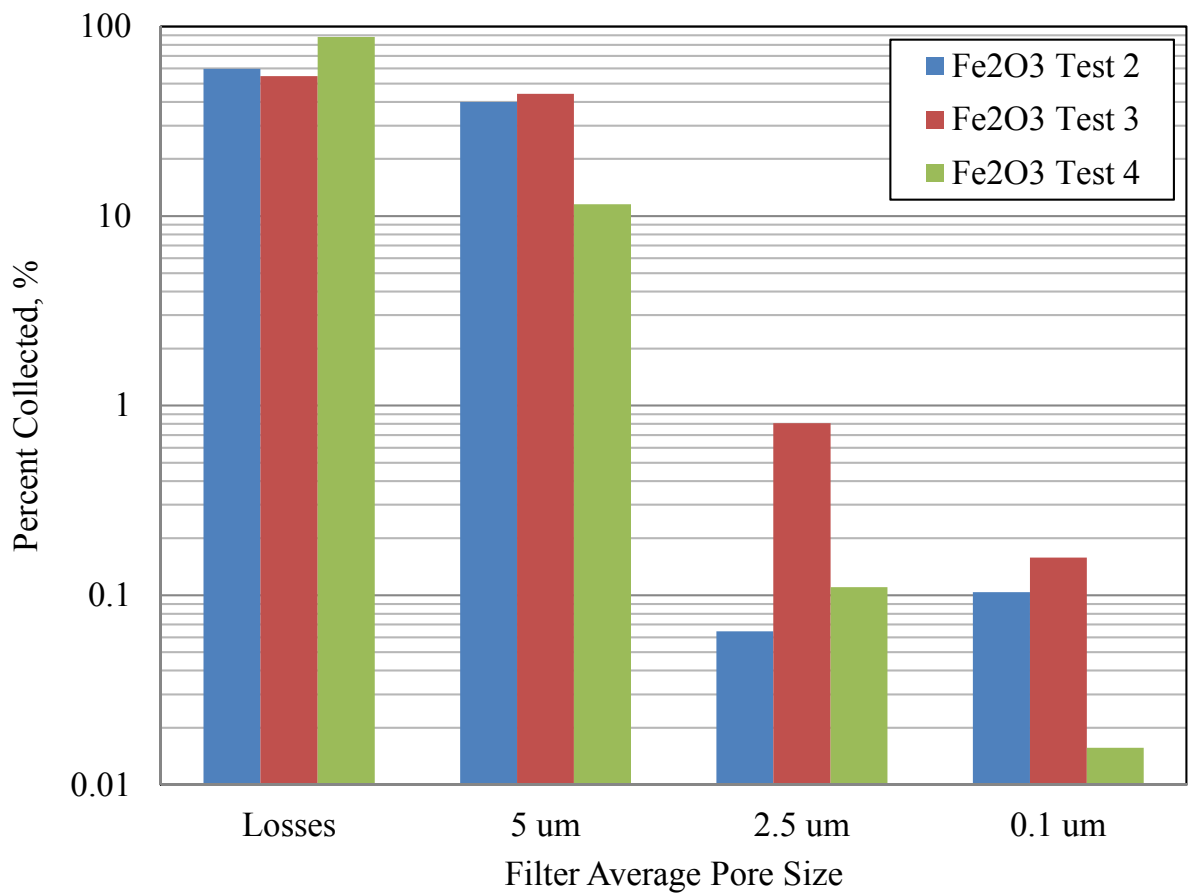

Figure 19: Iron oxide filter test percent collected results.

\subsubsection{Tungsten Carbide}

A tungsten carbide iron oxide aerosol stream was also evaluated using the Phase I 3-stage testing configuration. A baseline of $50 \mathrm{mg}$ was used as the loading mass while the minimum injection pressure was increased to $30 \mathrm{psig}$. The test specifications are provided in Table 7. The injection pressure and mass loaded were varied to determine the acceptable operating parameters. A dual loading and $300 \%$ run-time test were also conducted. The dual loading test consisted of running back-to-back tests without changing the filters to determine if a second injection would result in more material in the filters. Similarly, a $300 \%$ run-time test was performed to determine if longer test duration resulted in more captured particles.

Table 7: Tungsten carbide filter test data.

\begin{tabular}{|c|c|c|c|c|c|}
\hline $\begin{array}{c}\text { Test } \\
\text { Number }\end{array}$ & $\begin{array}{c}\text { Injection } \\
\text { Time } \\
(\mathrm{min})\end{array}$ & $\begin{array}{c}\text { Injection } \\
\text { Pressure } \\
(\mathrm{psig})\end{array}$ & $\begin{array}{c}\text { Mass } \\
\text { Loaded } \\
(\mathrm{g})\end{array}$ & $\begin{array}{c}\text { Mass } \\
\text { Injected } \\
(\mathrm{g})\end{array}$ & $\begin{array}{c}\text { Injection } \\
\text { Efficiency } \\
(\%)\end{array}$ \\
\hline 3 & 10 & 30 & 0.047 & 0.013 & 28 \\
\hline 4 & 10 & 30 & 0.010 & 0.006 & 60 \\
\hline 5 & 10 & 30 & 0.999 & 0.906 & 91 \\
\hline 7 & 10 & 49 & 0.049 & 0.017 & 35 \\
\hline 8 & $20^{(\mathrm{a})}$ & 30 & $0.102^{(\mathrm{a})}$ & $0.038^{(\mathrm{a})}$ & 37 \\
\hline 9 & 30 & 30 & 0.049 & 0.009 & 18 \\
\hline
\end{tabular}

(a) This test involved two 10 minute injections, each starting with 0.051 grams. A total of 0.038 grams were injected for the two tests. 
The WC mass and percent collected results are shown in Figure 20 and Figure 21, respectively. The DL was considerably better for tungsten, as compared to iron, and all measured masses were at least an order of magnitude above the detection limit. The comparison between $\mathrm{Fe}_{2} \mathrm{O}_{3}$ and $\mathrm{WC}$ filter tests, for identical experimental parameters (i.e., loaded mass, injection pressure, etc.) are shown in Figure 22 and Figure 23.

The results of the WC filter tests generated the following conclusions:

- Small particles within desired size range can be delivered to graphite samples

- The mass of small particles is measurable and can be quantified

- Higher losses than $\mathrm{Fe}_{2} \mathrm{O}_{3}$

o Attributed to the higher particle density

- Mass collected reduced for identical experimental parameters for WC versus $\mathrm{Fe}_{2} \mathrm{O}_{3}$

o Density impacts appear to be significant

0 WC filter results may be more appropriate for $\mathrm{UO}_{2}$ predictions than $\mathrm{Fe}_{2} \mathrm{O}_{3}$

- Further injection pressure increase produces smaller particles

- Increased mass loadings appear to reduce the quantity collected on the filters

o Possibly caused by large quantity of material blocking small diameter introduction fitting

o $50 \mathrm{mg}$ loading mass recommended for all subsequent tests

- Dual loading and $300 \%$ run time increase mass on 2.5 and $0.1 \mu \mathrm{m}$ filters

o Standard loading and 10 minute run-time provide measurable quantities on 2.5 and 0.1 $\mu \mathrm{m}$ filters. Dual loading and longer run times could be used if higher loadings were necessary.

- Slight injection efficiency improvement relative to $\mathrm{Fe}_{2} \mathrm{O}_{3}$

o More material being aerosolized 


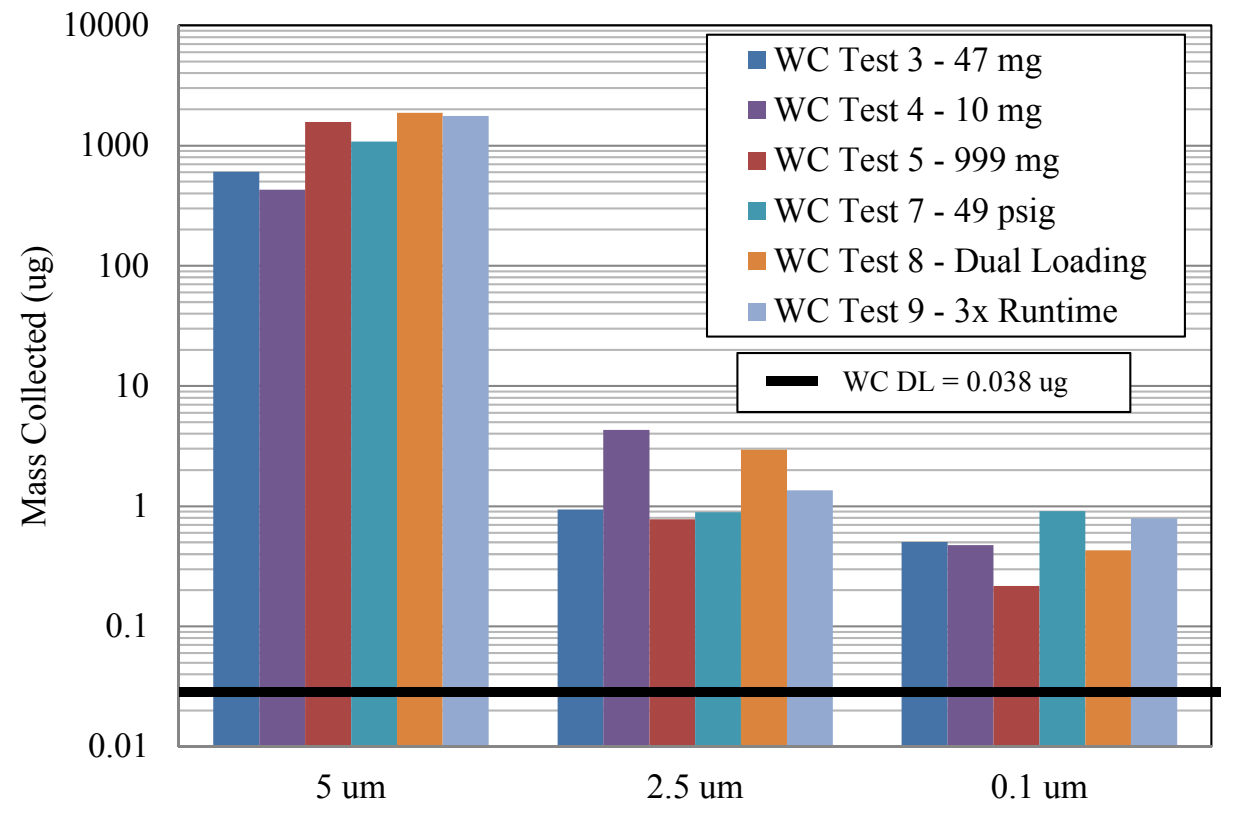

Filter Average Pore Size

Figure 20: Tungsten carbide filter test mass collected results.

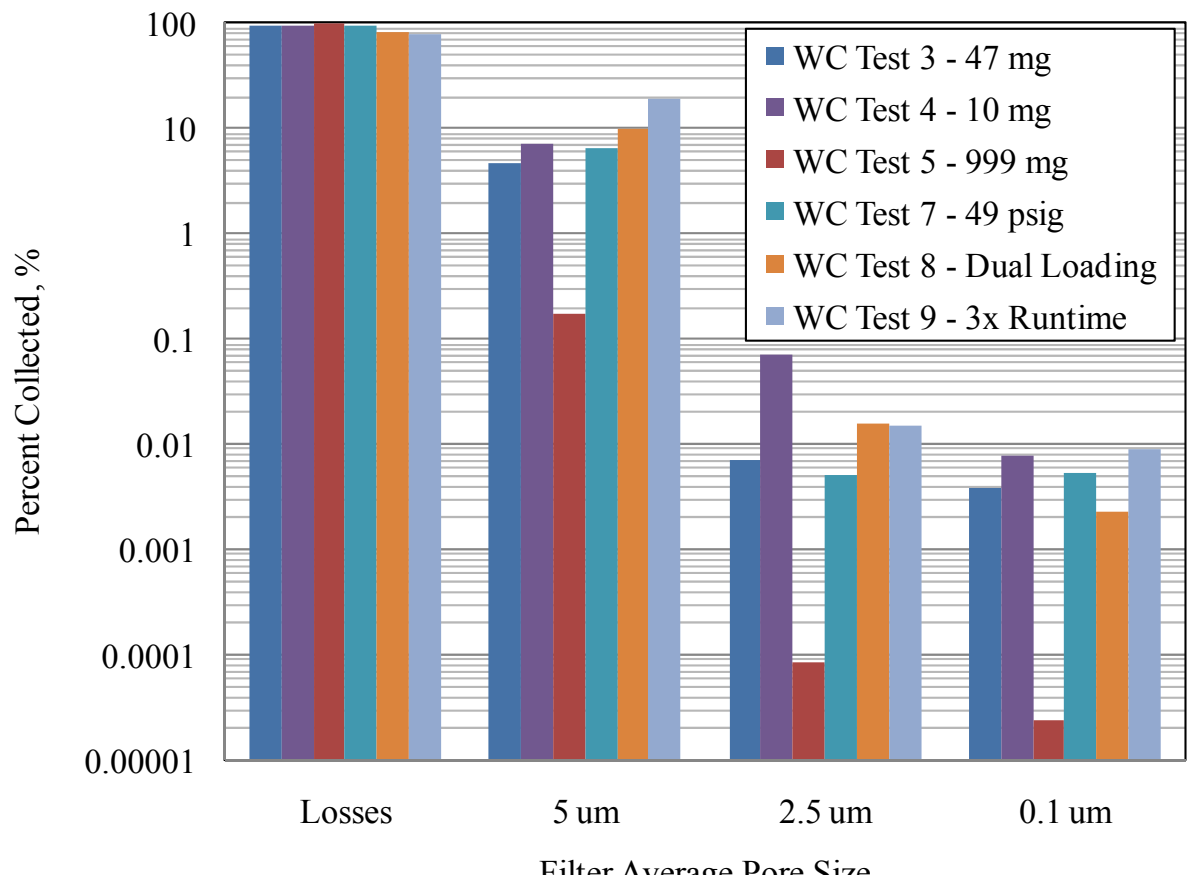

Figure 21: Tungsten carbide filter test percent collected results. 


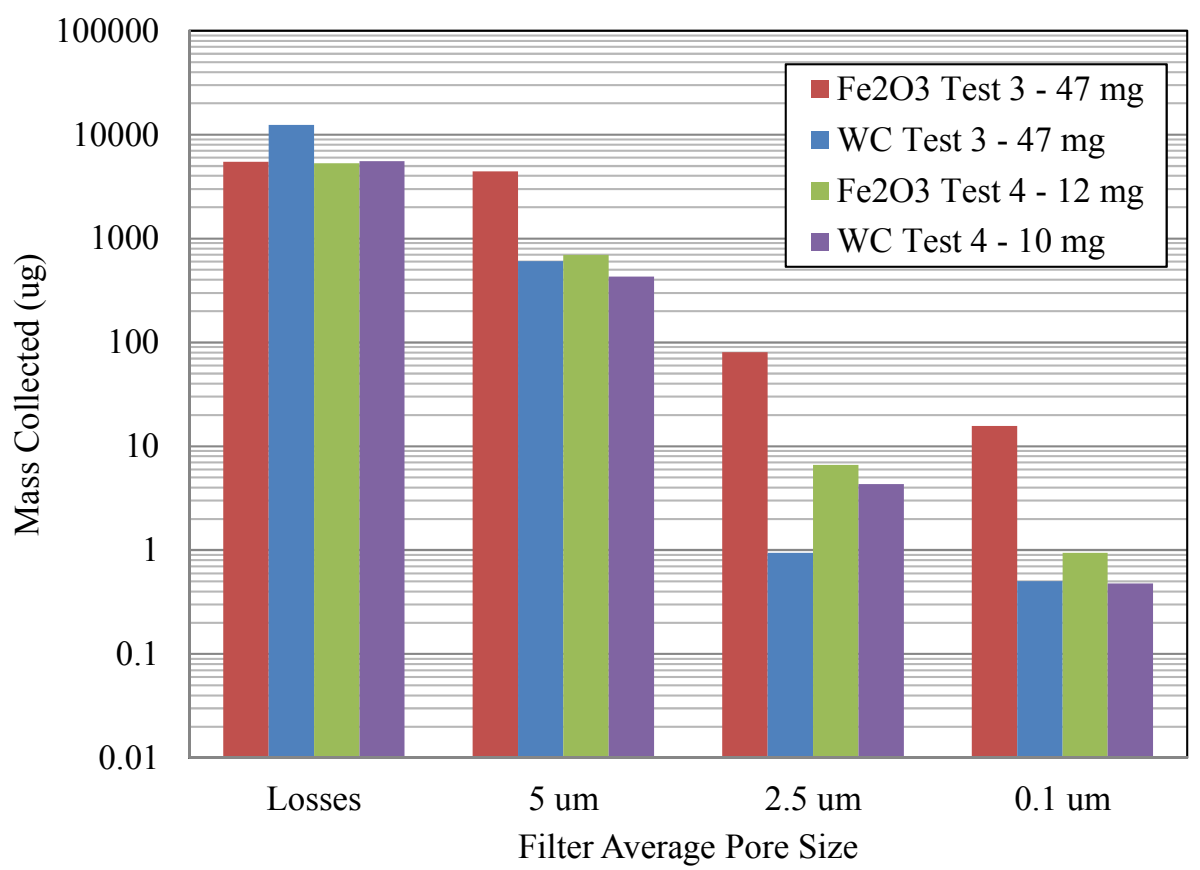

Figure 22: Iron oxide and tungsten carbide mass collected filter test comparison.

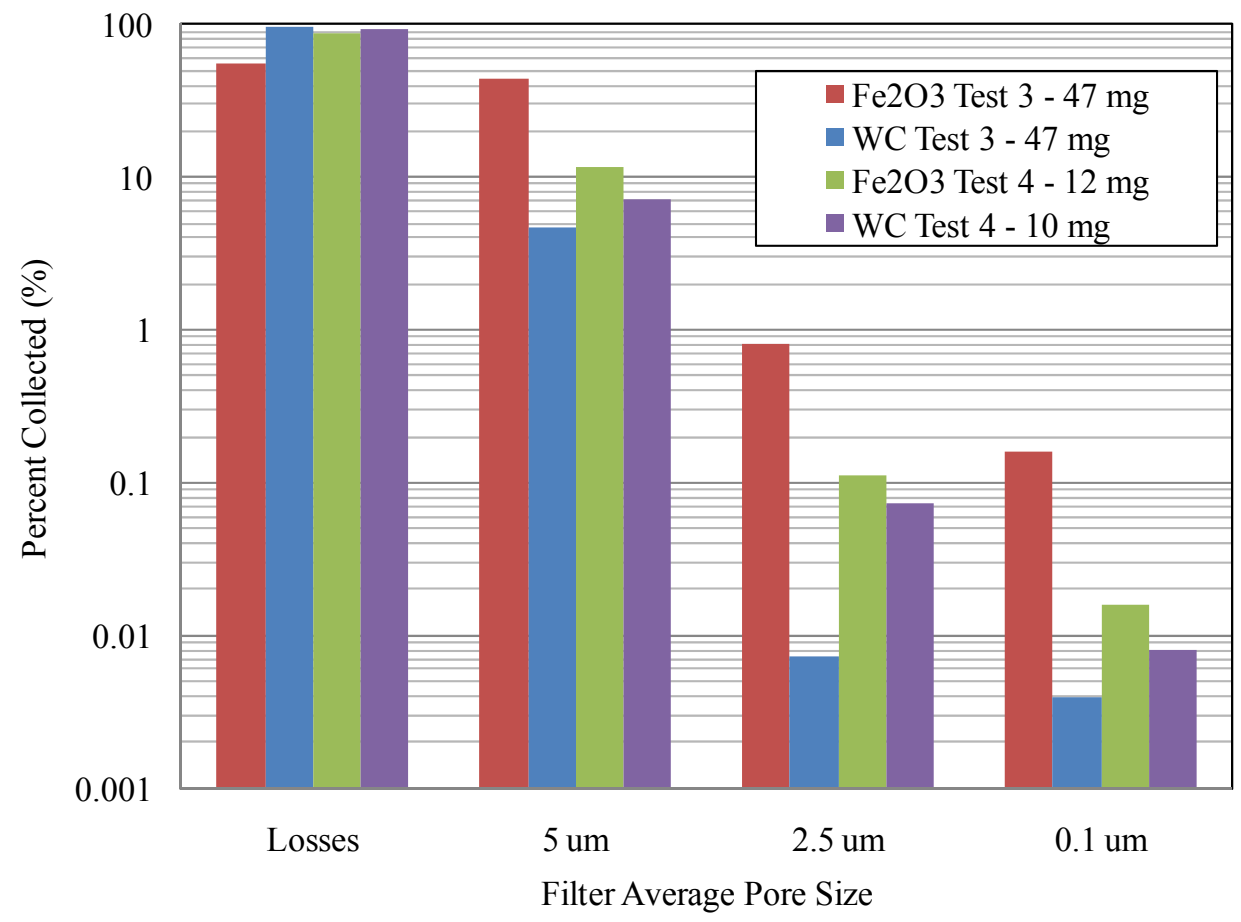

Figure 23: Iron oxide and tungsten carbide percent collected filter test comparison. 


\subsubsection{Uranium Oxide}

A single $\mathrm{UO}_{2}$ filter test was performed using the Phase II 3-stage testing configuration, prior to injecting graphite samples, to measure the mass of particles expected to be delivered to the graphite. It should be noted that the loading tee was not used for $\mathrm{UO}_{2}$ tests, rather the material was loaded into an elbow and placed just downstream of the pressure chamber, see Appendix E, Figure 53. The isolation and pressure relief valve were re-located appropriately. This loading configuration significantly increased the injection efficiency to approximately $97 \%$, as compared to $\sim 20$ and $\sim 30 \%$ for $\mathrm{Fe}_{2} \mathrm{O}_{3}$ and $\mathrm{WC}$ filter tests, respectively.

The $\mathrm{UO}_{2}$ filter test parameters are summarized in Table 8 and the results are shown in Figure 24, relative to similar test results for $\mathrm{Fe}_{2} \mathrm{O}_{3}$ and WC. The loaded mass in all three filter tests was approximately 50 $\mathrm{mg}$, however, the $\mathrm{UO}_{2}$ test used an injection pressure of 49 psig, compared to 30 psi for the $\mathrm{Fe}_{2} \mathrm{O}_{3}$ and WC tests. The UDL for uranium was much better than either iron or tungsten resulting in two orders of magnitude between the lowest measured mass and the UDL.

Table 8: Uranium Oxide filter test data.

\begin{tabular}{|c|c|c|c|c|}
\hline $\begin{array}{c}\text { Test } \\
\text { Number }\end{array}$ & $\begin{array}{c}\text { Injection } \\
\text { Pressure } \\
(\mathrm{psig})\end{array}$ & $\begin{array}{c}\text { Mass } \\
\text { Loaded } \\
(\mathrm{g})\end{array}$ & $\begin{array}{c}\text { Mass } \\
\text { Injected } \\
(\mathrm{g})\end{array}$ & $\begin{array}{c}\text { Injection } \\
\text { Efficiency } \\
(\%)\end{array}$ \\
\hline 6 & 49 & 0.0544 & 0.0525 & 97 \\
\hline
\end{tabular}

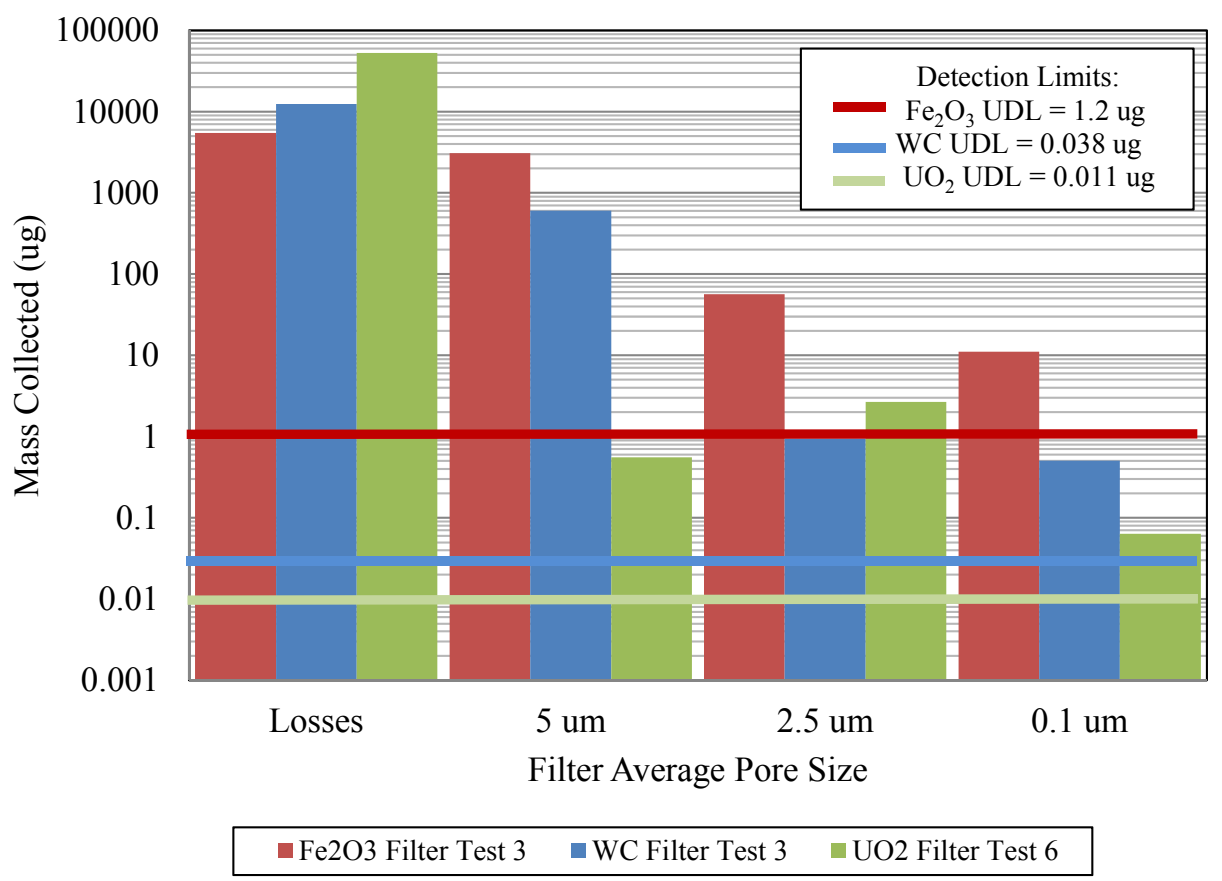

Figure 24: 3-stage filter test comparison for $\mathrm{Fe}_{2} \mathrm{O}_{3}$, WC and $\mathrm{UO}_{2}$.

As indicated in Figure 24, the mass of $\mathrm{UO}_{2}$ on all the filters was significantly lower than that of $\mathrm{Fe}_{2} \mathrm{O}_{3}$ which was expected based on $\mathrm{UO}_{2}$ higher density. However, the mass collected on the $0.1 \mu \mathrm{m}$ filter was 
much lower than the mass of WC collected but the total mass collected on both the 2.5 and $0.1 \mu \mathrm{m}$ filters was much higher for the $\mathrm{UO}_{2}$ compared to the WC tests. The particle size distribution of the $\mathrm{UO}_{2} \mathrm{was}_{\mathrm{W}}$ not measured but these results indicate that the $\mathrm{UO}_{2}$ might have a significant fraction of particles below $5 \mu \mathrm{m}$ but fewer particles less than $2.5 \mu \mathrm{m}$.

The concentration assuming that the mass collected on the 2.5 and $0.1 \mu \mathrm{m}$ filters (i.e., that which was expected to be delivered to the graphite sample) was homogeneously distributed in a $5 \mathrm{~cm}$ thick graphite cylinder is summarized in Table 9, for each material tested. The three tests using similar experimental parameters are highlighted for clarity. The $\mathrm{UO}_{2}$ filter test predicted a concentration of $51 \mathrm{ppb}$ of $\mathrm{UO}_{2}$ in the graphite sample. The simplifying homogeneous distribution assumption showed that the quantity of $\mathrm{UO}_{2}$ delivered to the graphite surface should be readily detectable. The $\mathrm{UO}_{2}$ was expected to be concentrated near the surface due to particle capture mechanisms so the mass of $\mathrm{UO}_{2}$ expected to be delivered to the surface of the graphite should result in even higher concentrations.

Table 9: Concentration of material homogeneously distributed in a $5 \mathrm{~cm}$ thick graphite cylinder.

\begin{tabular}{|c|c|c|c|c|}
\hline $\begin{array}{c}\text { Test } \\
\text { Number }\end{array}$ & $\begin{array}{c}\text { Mass } \\
\text { Loaded }\end{array}$ & $\begin{array}{l}\text { Injection } \\
\text { Pressure }\end{array}$ & $\begin{array}{c}\text { Mass Collected } \\
(2.5 \text { and } 0.1 \mu \mathrm{m} \text { filters })\end{array}$ & Concentration \\
\hline & (g) & (psig) & $(\mu \mathrm{g})$ & (ppb) \\
\hline \multicolumn{5}{|c|}{$\mathrm{Fe}_{2} \mathrm{O}_{3}$} \\
\hline 2 & 0.043 & 10 & 11.75 & 261 \\
\hline 3 & 0.047 & 30 & 96.42 & 2143 \\
\hline 4 & 0.012 & 30 & 7.58 & 168 \\
\hline \multicolumn{5}{|c|}{$\mathrm{WC}$} \\
\hline 3 & 0.047 & 30 & 1.45 & 32 \\
\hline 4 & 0.010 & 30 & 4.80 & 107 \\
\hline 5 & 0.999 & 30 & 0.99 & 22 \\
\hline 7 & 0.049 & 49 & 1.80 & 40 \\
\hline \multicolumn{5}{|c|}{$\mathrm{UO}_{2}$} \\
\hline 6 & 0.054 & 49 & 2.30 & 51 \\
\hline
\end{tabular}




\subsection{Graphite Injection Results}

\subsection{Sample Preparation}

Three graphite samples were subjected to $\mathrm{UO}_{2}$ aerosol flow tests using the ATS. Prior to analyzing the graphite, the potted samples were cut opened and the shrink wrap discarded. The outside surface of the sample was carefully machined using a lathe to remove the graphite containing any shrink wrap adhesive, see Figure 25. Each sample was then cut into 6 slices (or discs) per Figure 25 and Table 10. Note, the actual slice thickness varied slightly. The thickness of the slices varied over the length of the sample based on the expected concentration profile. For a single particle size, the particle concentration profile would be expected to decrease exponentially with penetration depth which would result in a straight line when plotted as log concentration versus depth. However, when a distribution of particle sizes is introduced to the surface of the graphite sample, each size would have its own exponential penetration curve, with particles larger and smaller than the maximally penetrating size depositing nearer to the surface. Therefore, the expected concentration profile (when plotted as log concentration versus depth) would be steepest at the surface of the graphite but become less steep and linear as all but the maximally penetrating sized particles are filtered out. Therefore, the slice thickness dimensions were chosen to better resolve the expected penetration profiles by taking thinner slices at the front face of the graphite.

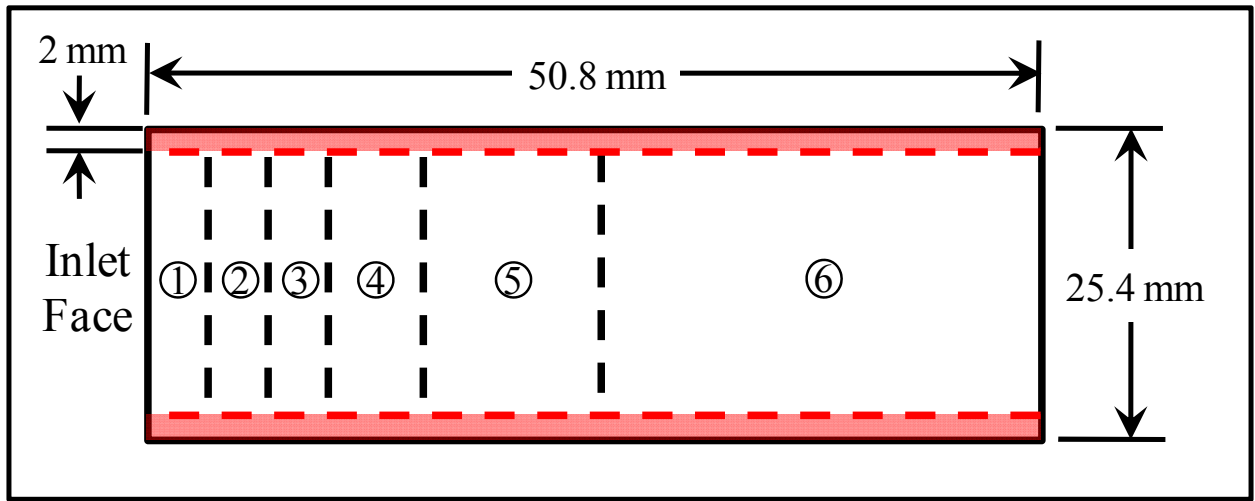

Figure 25: Graphite injection sample preparation and slicing protocol. Note that $\sim 2 \mathrm{~mm}$ were machined from the outer surface of the graphite prior to slicing operations.

Table 10: Graphite injection sample nominal slicing dimensions.

\begin{tabular}{|c|c|c|}
\hline $\begin{array}{c}\text { Slice } \\
\text { Number }\end{array}$ & $\begin{array}{c}\text { Nominal Slice } \\
\text { Thickness }(\mathrm{mm})\end{array}$ & $\begin{array}{c}\text { Nominal Slice } \\
\text { Midpoint }(\mathrm{mm})\end{array}$ \\
\hline 1 & 2 & 1 \\
\hline 2 & 2 & 3 \\
\hline 3 & 2 & 5 \\
\hline 4 & 4 & 8 \\
\hline 5 & 8 & 14 \\
\hline 6 & 32.8 & 34.4 \\
\hline
\end{tabular}




\subsection{Analysis Technique}

Each graphite slice was ashed (i.e., process of burning graphite and reducing to an ash) in either a tube or chamber furnace, with a low flow rate of pure oxygen, to accelerate combustion. The resulting ash was carefully collected and digested in an acidic solution. A known amount of $U_{233}$ tracer was added to each solution and analyzed using an ICP-MS. The analysis yielded uranium isotope ratios (i.e., $\mathrm{U}_{233} / \mathrm{U}_{238}$, $\mathrm{U}_{236} / \mathrm{U}_{238}$, etc.) and from the starting $\mathrm{U}_{233}$ mass, the mass of each isotope was calculated. This measurement and analytical technique results in an extremely accurate uranium isotopic ratios and calculated masses with low detection limits.

\subsection{Uranium Oxide Results}

\subsubsection{ATS Testing Data}

The concentration profile of $\mathrm{U}_{236}$ isotope is shown in Figure 26 for the three graphite samples. The instrument detection limit associated with the $U_{236}$ isotope was approximately $10^{-6} \mathrm{ppb}$, two orders of magnitude below the lowest measurement of $10^{-4} \mathrm{ppb}$. This profile shows that $\mathrm{U}_{236}$, which can only come from the introduced $\mathrm{UO}_{2}$ particles, is penetrating to distances of at least $35 \mathrm{~mm}$. The profile was, as expected, steepest near the surface of the graphite, becoming less steep and more linear at greater penetration depths.

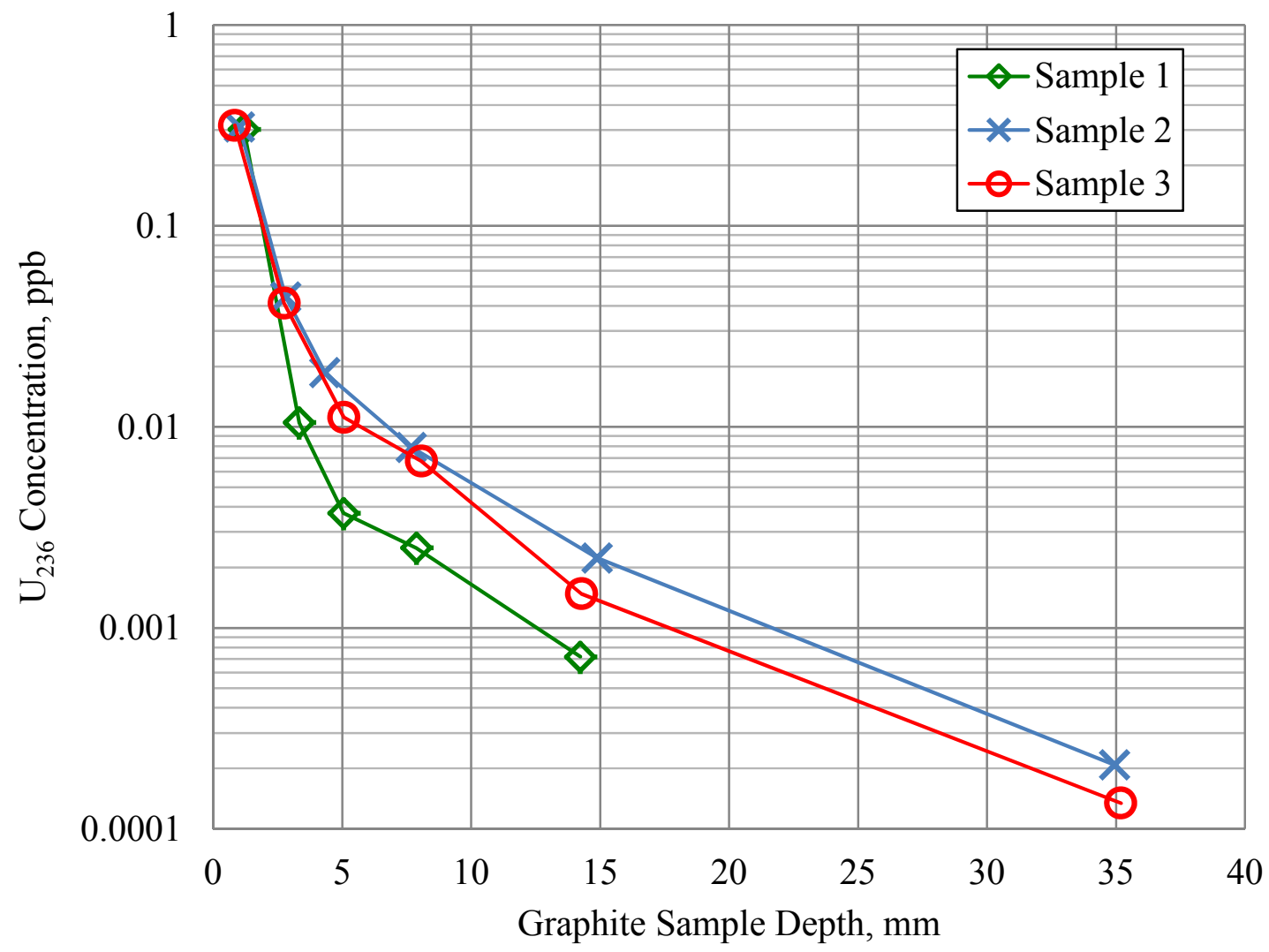

Figure 26: $U_{236}$ isotope concentration profile in graphite samples. 
The $\mathrm{UO}_{2}$ penetration profile was calculated from the $\mathrm{U}_{236}$ profile and the known composition of the Redding Enriched Uranium (EU) (see Table 3). This calculation method eliminated the need to account for a large and potentially variable background of $\mathrm{UO}_{2}$ in the graphite samples. The resulting profile (Figure 27) shows measured $\mathrm{UO}_{2}$ concentrations of up to $10 \mathrm{ppb}$ (approximately equal to the expected background levels in reactor graphite) at depths of $15 \mathrm{~mm}$. Sample 2 and 3 exhibited similar $\mathrm{UO}_{2}$ profiles but the profile for sample 1 was significantly lower. This was most likely attributed to the lower injected mass (roughly $40 \%$ less than sample 2 and 3); however, the permeability of the graphite may have also reduced the flow rate and $\mathrm{UO}_{2}$ penetration distances. The permeability and calculated flow rate at 10 psi for all three graphite samples are provided in Error! Reference source not found.

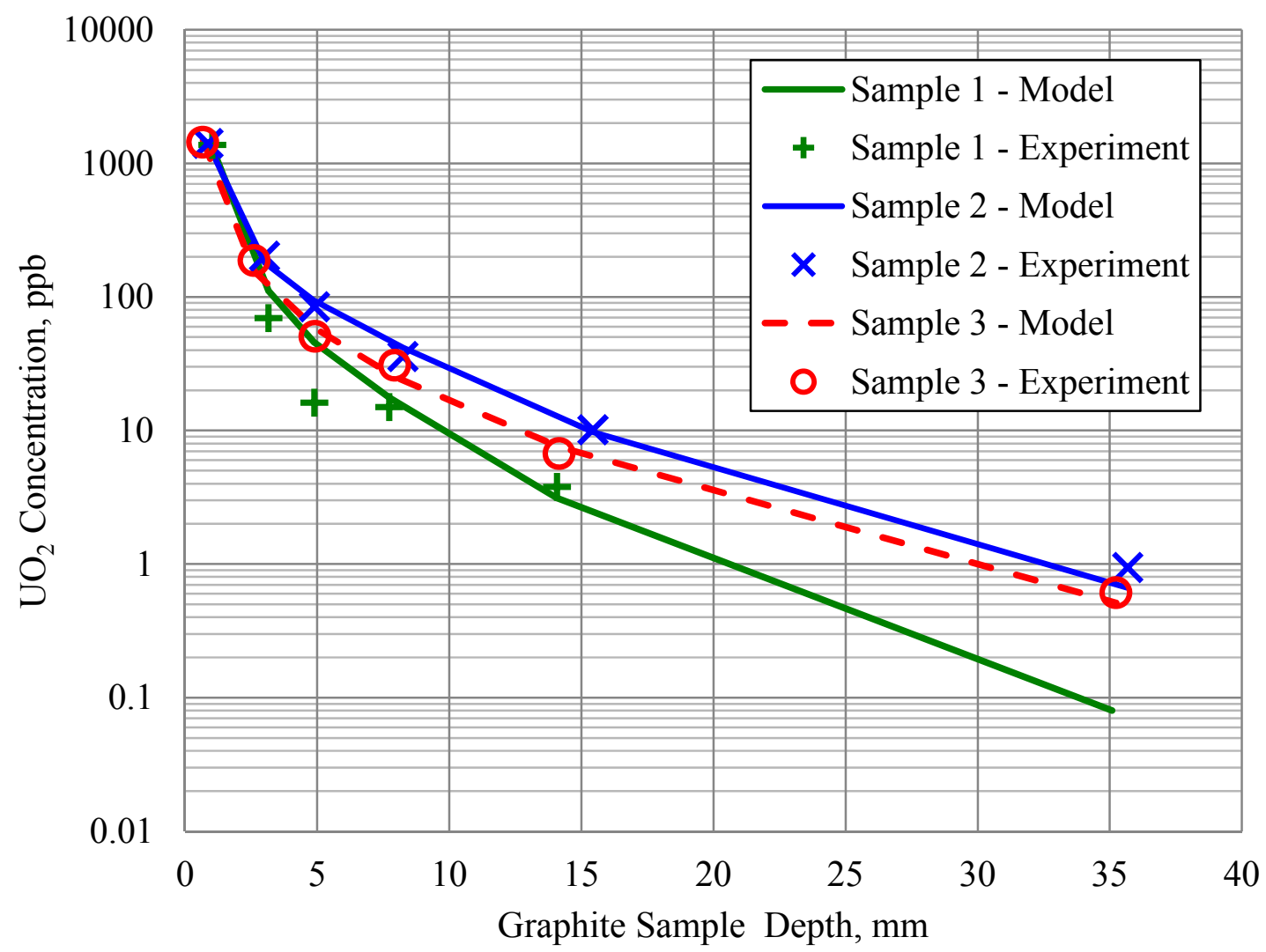

Figure 27: ATS experiment and unit collector model comparison of $\mathrm{UO}_{2}$ concentration profile.

Table 11: $\mathrm{UO}_{2}$ injection graphite permeability and flow rate summary

\begin{tabular}{|c|c|c|}
\hline $\begin{array}{c}\text { Graphite } \\
\text { Sample }\end{array}$ & $\begin{array}{c}\text { Permeability } \\
\text { (md) }\end{array}$ & $\begin{array}{c}\text { Flow rate @ } 10 \mathrm{psi} \\
\text { (cc/s) }\end{array}$ \\
\hline 1 & 185 & 9.1 \\
\hline 2 & 192 & 9.5 \\
\hline 3 & 193 & 9.5 \\
\hline
\end{tabular}

A mass balance was performed to double check the $\mathrm{UO}_{2}$ concentrations measured in the graphite samples. The first step of this mass balance was to estimate the mass of $\mathrm{UO}_{2}$ that was delivered to the surface of 
the graphite samples. The $5 \mu \mathrm{m}$ pre-filter from each graphite injection test was analyzed using ICP-MS and the results are shown in Error! Reference source not found., along with the results for the $\mathrm{UO}_{2}$ filter test. The excellent agreement of the results from the $5 \mu \mathrm{m}$ pre-filter fully supports an assertion that the amount of $\mathrm{UO}_{2}$ delivered to the graphite samples in the injection tests is the same as the total combined mass collected on the 2.5 and $0.1 \mu \mathrm{m}$ filters in the filter test. Thus, the mass collected on the second $\left(\mathrm{F}_{2}\right)$ and third $\left(\mathrm{F}_{3}\right)$ filters from filter test 6 provided a means to make a rough estimate of how much $\mathrm{UO}_{2}$ mass was introduced to the surface of the graphite samples in the graphite injection tests.

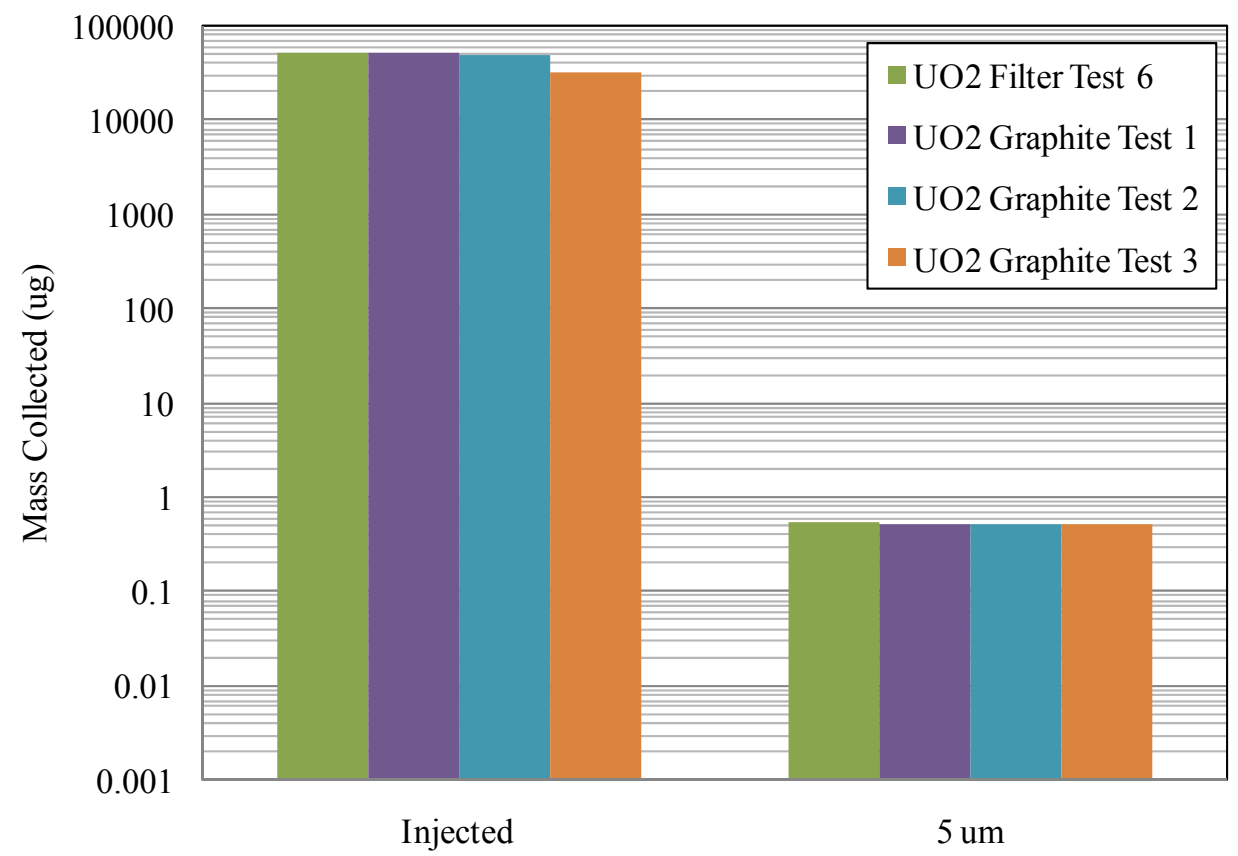

Filter Average Pore Size

Figure 28: Mass collected on $5 \mu \mathrm{m}$ filters in $\mathrm{UO}_{2}$ graphite injection and filter tests

Table 12 provides a summary of $\mathrm{UO}_{2}$ masses measured from filter test 6 and the three graphite tests shown in Figure 27. The mass balance was performed by estimating the total mass of $\mathrm{UO}_{2}$ in the graphite by adding the mass in all six slices that were determined from the $\mathrm{UO}_{2}$ concentrations and masses in each slice. The total mass in the graphite was then compared to the estimated introduced mass to determine a rough mass balance. The calculated total $\mathrm{UO}_{2}$ mass for each graphite sample was 50 to $80 \%$ of the introduced mass indicating a reasonable mass balance between the expected and measured mass of $\mathrm{UO}_{2}$ captured by the graphite. This rough mass balance supports the concentrations measured in the graphite samples.

A calculation was performed to get a sense for the number of particles that penetrated deeply into the graphite. The calculation converted the mass of $\mathrm{UO}_{2}$ measured in the deepest segment (segment 6) into a number of spherical particles with an assumed particle size of $350 \mathrm{~nm}$. This size was assumed because it was the expected maximally penetrating particle from Figure 30 as discussed in the following section. The calculation indicates that $>50,000$ particles penetrate a minimum of $18 \mathrm{~mm}$ into the deepest segment of graphite sample. 
Table 12: Summary of $\mathrm{UO}_{2}$ mass measurements in filter and graphite tests.

\begin{tabular}{|c|c|c|c|c|c|c|}
\hline Test & $\begin{array}{c}\text { Injected } \\
\text { Mass } \\
(\mathrm{mg})\end{array}$ & $\begin{array}{c}5 \mu \mathrm{m} \\
\text { Filter } \\
\text { Mass } \\
(\mu \mathrm{g})\end{array}$ & $\begin{array}{c}\text { Filter Test 6 } \\
\text { Summed } \\
\mathrm{F}_{2} \text { and } \mathrm{F}_{3} \\
\text { Masses }(\mu \mathrm{g})\end{array}$ & $\begin{array}{c}\text { Measured } \\
\text { Mass in } \\
\text { Graphite } \\
(\mu \mathrm{g})\end{array}$ & $\begin{array}{c}\text { Estimated } \\
\text { Percent } \\
\text { Recovery }\end{array}$ & $\begin{array}{c}\text { Calculated } \\
\text { Number of } \\
350 \mathrm{~nm} \\
\text { Particles } \\
\text { Present in } \\
\text { Segment 6 }\end{array}$ \\
\hline $\begin{array}{c}\text { Filter } \\
\text { Test 6 }\end{array}$ & 52.53 & 0.553 & 2.732 & N/A & N/A & N/A \\
\hline $\begin{array}{c}\text { Graphite } \\
\text { Sample 1 }\end{array}$ & 32.66 & 0.517 & N/A & 1.374 & $50 \%$ & $\mathrm{NM}^{(\mathrm{c})}$ \\
\hline $\begin{array}{c}\text { Graphite } \\
\text { Sample 2 }\end{array}$ & 49.50 & 0.514 & N/A & 2.180 & $80 \%$ & 77,254 \\
\hline $\begin{array}{c}\text { Graphite } \\
\text { Sample 3 }\end{array}$ & 51.49 & 0.515 & N/A & 1.730 & $63 \%$ & 53,039 \\
\hline
\end{tabular}

(a) The percent recovery assumed 2.732 ug of $\mathrm{UO}_{2}$ passed through the $5 \mu \mathrm{m}$ pre-filter and was introduced to the surface of the graphite.

(b) Calculation assumes $350 \mathrm{~nm}$ as the expected maximum penetrating particle from Figure 30.

(c) NM indicates not measured.

\subsubsection{Tuned Unit Collector Model}

The $\mathrm{UO}_{2}$ penetration results from the final ATS experiments were used to determine the representative collector size (i.e., filtration length scale) for the unit collector model described in Section 3.1. The first step of this process was to make reasonable assumptions about the particle size distribution of the $\mathrm{UO}_{2}$ particles that were introduced to the surface of the graphite sample since this distribution was not measured. Log-normal size distributions are often encountered in natural systems, and are described by two parameters: the mean and standard deviation of the natural logarithm of the particle diameter. Although the inlet size distribution is not known, and probably does not correspond exactly to a lognormal distribution, the real distribution would likely include some of the same features. A realistic distribution is likely a smooth curve approximately centered around a mean value, with 'tails' in the direction of very large and very small particles. While the tail on the large diameter end of the distribution contains relatively few particles, it contains a significant fraction of the mass, since particle mass is a function of the diameter cubed. Since the particle stream first passed through a $5 \mu \mathrm{m}$ pre-filter, the log-normal distribution was truncated on the large end. In order to describe the ATS data, the assumed inlet PSD mean and standard deviation were tuned to 0.44 and $0.5 \mu \mathrm{m}$, respectively, and the distribution was truncated at $3 \mu \mathrm{m}$. Note that the model is relatively insensitive to the exact inlet PSD assumed when used to fit the mass-based data from the ATS experiments, as long as there are some particles at the large end of the distribution to be trapped at relatively shallow depths in the graphite. The assumed inlet PSD is shown on a mass basis in Figure 29. 
PNNL-21014

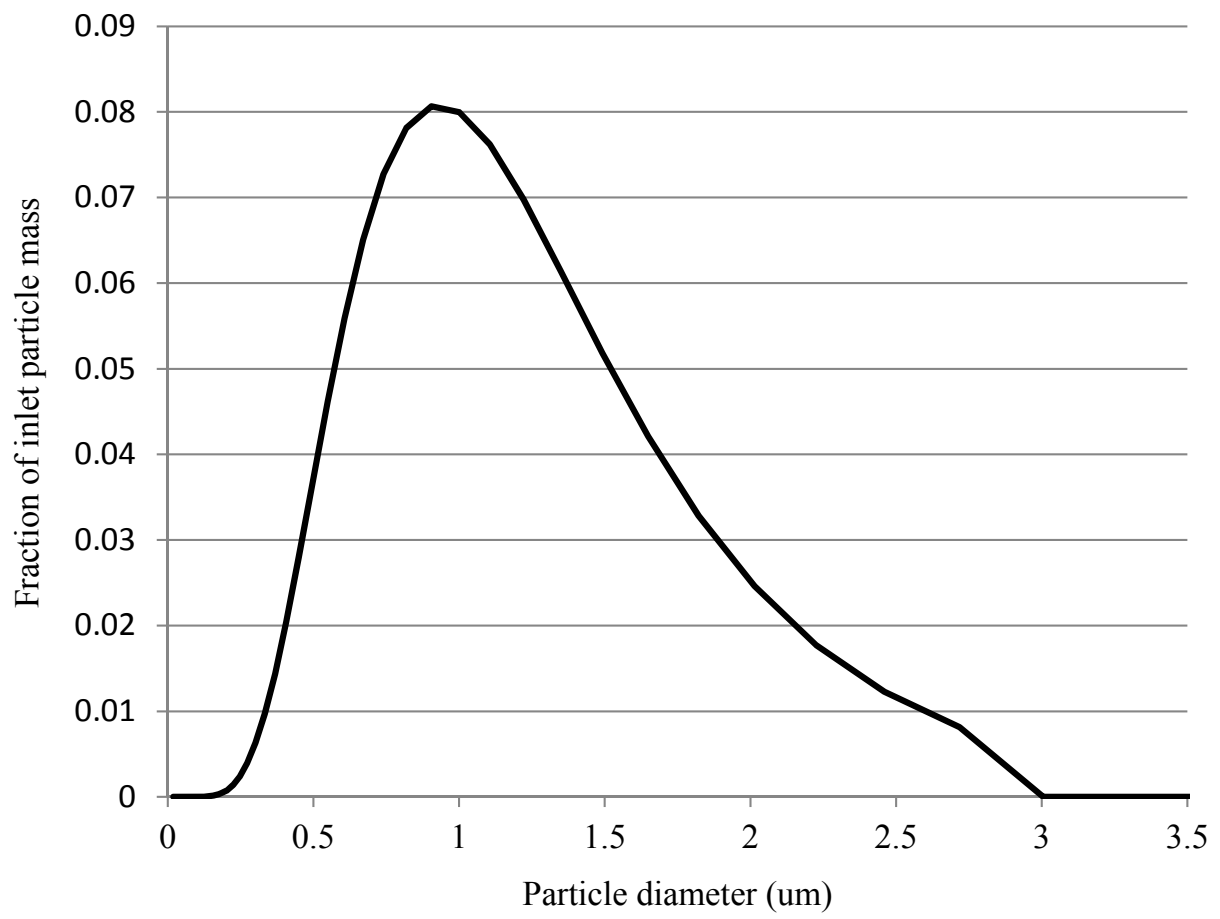

Figure 29: Unit collector model inlet mass fraction.

In order to fit the $\mathrm{UO}_{2}$ penetration data, the representative collector size (i.e., filtration length scale) was adjusted. A good fit of the data was obtained by using collector sizes of $110 \mu \mathrm{m}$ for Sample 1, and 140 $\mu \mathrm{m}$ for Samples 2 and 3. The different values are consistent with the fact that the measured permeabilities of Samples 2 and 3 were similar at 192 and $193 \mathrm{mD}$ while the permeability of Sample 1 was significantly lower at $185 \mathrm{mD}$. The collector diameter determined from the fitted data was more than twice as large as the preliminary estimates that were made based on the permeability and assumed representative pore size. The higher collector diameters indicted from the empirical data significantly change the model and lead to predictions of dramatically deeper particle penetration and a somewhat larger maximally penetrating particle diameter, as shown in Figure 30. 
PNNL-21014

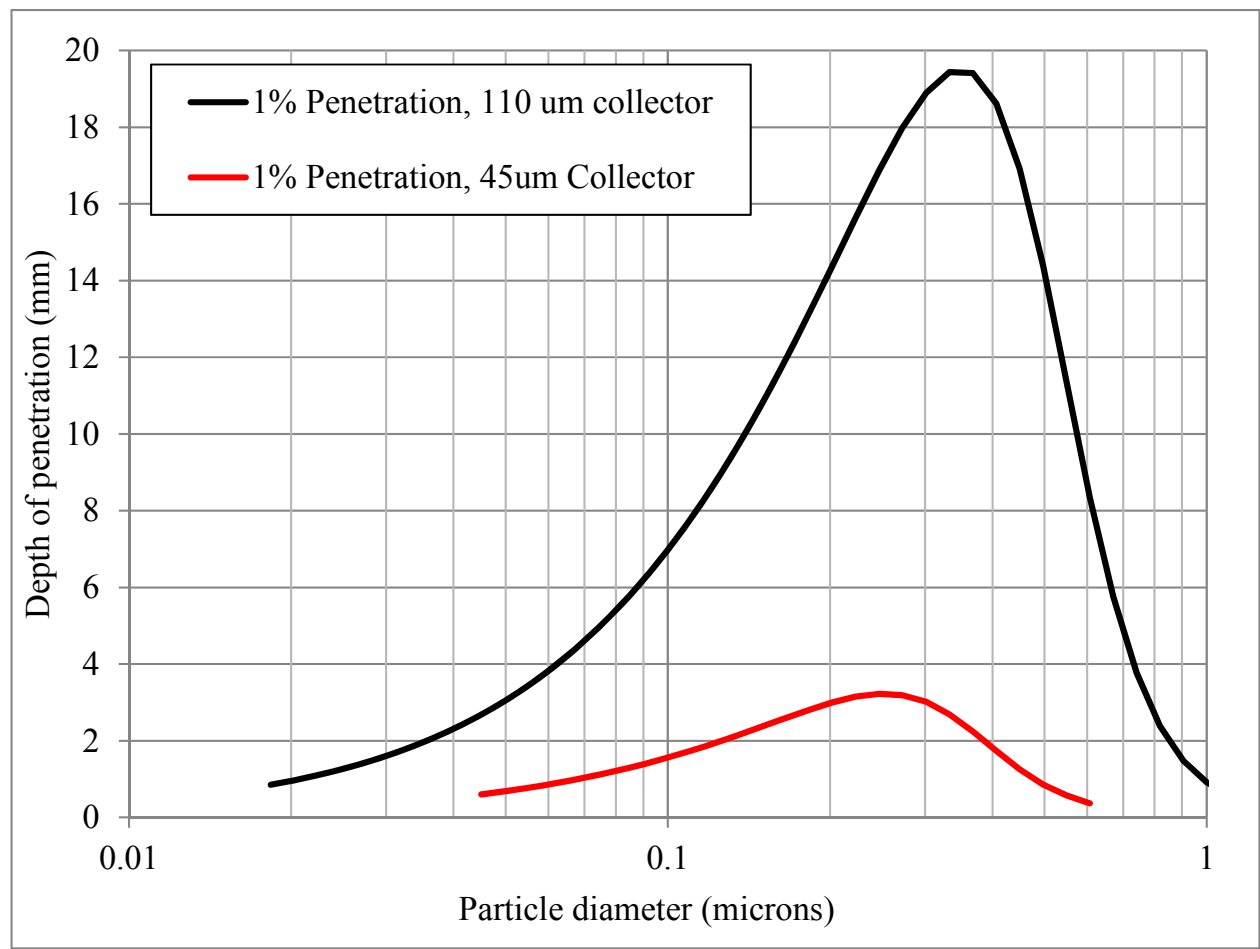

\section{Figure 30: Comparison of particle penetration predicted by the preliminary and tuned unit collector models.}

It retrospect, it is not surprising that the relationship between representative collector diameter and pore size/permeability in a pack of spheres does not translate well to a graphite filter material. The graphite microstructure shown in Figure 33 is clearly much different than that of a bed of spheres or any other loose particles. Most of the void volume in randomly packed bed of particles is characterized by a tortuous series of highly connected pore bodies and throats with dimensions on the order of the particle diameter. One interpretation of the larger representative collector size needed to match the ATS data is that the connected porosity in graphite is arranged in channels that extend further through the graphite medium than similar sized pores would extend through a spherical particle pack with a similar permeability.

It appears that, while there is some relationship between permeability and representative collector size, it is not the same as the loose, granular bed relationship. Also, the model results depend, to some extent, on the inlet particle size distribution, which was not measured in these experiments. Therefore, it is not recommended that the proposed unit collector model be used to make quantitative predictions for $\mathrm{UO}_{2}$ penetration into graphite under other conditions without further validation. Nevertheless, the model provides a framework to understand the fundamental physics involved in graphite filtration and provides a good description of the data collected thus far when reasonable assumptions are made. The model could be used to provide qualitative estimates of how $\mathrm{UO}_{2}$ penetration would be expected to change as a function of parameters such as flow rate, particle size distribution, particle and fluid properties, etc.

If a modeling capability were important, a more extensive data set would allow for validation of the modeling approach and a better definition of the relationship between permeability and filter efficiency for a given type of graphite. If enough samples were examined, the extent of variation in representative collector size might also be used to make bounding estimates of the extent of penetration as a function of 
PNNL-21014

particle size. Experiments which measure the particle size distribution introduced to the surface of the graphite sample would also help reduce modeling uncertainty. 
PNNL-21014

\subsection{Implications for GIRM Applications}

Graphite penetration tests indicate that it is possible for uranium from fuel failures to be transported by the carbon dioxide gas coolant system and deposited at depths as great as $35 \mathrm{~mm}$ in the graphite blocks of a graphite moderated reactor. Since a nominal GIRM graphite sample is taken within the first $19 \mathrm{~mm}$, uranium from fuel failures has the potential to adversely impact a GIRM assessment.

It should be noted that while it may be possible that uranium from fuel failures can be transported and incorporated into the graphite moderator blocks, there is no documented evidence in the literature that such circumstances have been observed in the hundreds of operating years of experience with such reactors. Furthermore, this work investigated a worst case scenario and constructed an environment that maximized the potential for transport of $\mathrm{UO}_{2}$ into graphite. If penetration distances had been low under these conditions, there would be little concern that uranium from fuel failures would interfere with GIRM analyses. The large penetration distances measured in these studies show that penetration is possible under optimum conditions, but there are several factors that could limit penetration distances in an actual graphite reactor environment. These factors include:

- Flow path geometry: The ATS flow path ensured that particles were introduced to the surface of the graphite sample. The particles in the actual reactor would be suspended in $\mathrm{CO}_{2}$ coolant traveling $20 \mathrm{~m} / \mathrm{s}$ in a direction perpendicular to the surface of the graphite. To penetrate into the graphite, the aerosol particles would need to turn $90^{\circ}$ into a stream traveling less than $10 \mathrm{~cm} / \mathrm{s}$. The required change in flow path direction might limit the quantity and size of particles that are introduced to the surface of the graphite and limit penetration.

- $\mathrm{UO}_{2}$ PSD generated in a graphite reactor: One of the first steps in this work was to predict the most penetrating $\mathrm{UO}_{2}$ particle size for a graphite material. The test conditions were then tailored to make sure that at least some particles with those dimensions were introduced to the surface of the graphite in quantities that would be readily detectable. The actual PSD of $\mathrm{UO}_{2}$ that is produced from fuel failures may be significantly different and less penetrating.

- $\mathrm{UO}_{2}$ deposition: The ATS ensured that $\mathrm{UO}_{2}$ was introduced to the graphite surface. In a graphite rector, other areas in the closed-loop flow through the reactor and mechanical systems (e.g., pump, heat exchangers, etc.) may act as natural collection points for any $\mathrm{UO}_{2}$ generated. If the bulk of the $\mathrm{UO}_{2}$ is removed in these other areas, it will not be available to penetrate into the graphite.

- Graphite characteristics: The surface of graphite samples used in the ATS were relatively clean and a $5 \mu \mathrm{m}$ pre-filter was used to prevent large masses of large particles/agglomerates from clogging the graphite surface pores. The graphite in a reactor would be exposed to dusts (graphite dust, large $\mathrm{UO}_{2}$ particles, etc.) that could fill the large pores and significantly reduce penetration. Also, the reactor graphite might 
have different physical properties (i.e., pore size, permeability, porosity, etc.) than the test PGX graphite which could impact filtration behavior.

The lack of any documented penetration in the literature and the previous points indicate that the penetration of uranium seen in these worst case tests may not be realized when the $\mathrm{UO}_{2}$ is generated from fuel failures in an actual reactor environment. Nonetheless, these tests show that steps must be taken to identify and ensure that uranium contamination from fuel failures does not adversely impact a GIRM assessment. A standard protocol to evaluate whether a GIRM graphite sample has been compromised by uranium from fuel failures should be implemented and appropriate steps should be taken to ensure that any compromised sample uses other indicator elements for GIRM assessment. 


\subsection{Conclusion}

The conclusions from this study are provided in the following bullets:

- Estimates of the amount of $\mathrm{UO}_{2}$ that might be generated from fuel failures over a reactor lifetime indicate the enough $\mathrm{UO}_{2}$ is generated to potentially impact graphite uranium impurity levels.

- The high flow velocities in a graphite reactor indicate that $\mathrm{UO}_{2}$ particles are readily transported throughout the reactor.

- Characterization of PGX graphite samples, that are thought to be representative of materials used in graphite moderated reactors, showed total porosities that ranged from $22-23 \%$ and permeabilities that ranged from 130-200 md. At $10 \mathrm{psig}$, the average pore velocity in these samples would be $\sim 7 \mathrm{~cm} / \mathrm{s}$. Most of the porosity was open porosity. About half of the open porosity was comprised of pore throats that were 1-20 microns with the other half comprised of pore throats that were less than 1 micron.

- A unit collector model estimated the worst-case, furthest penetrating aerosol particle size for PGX graphite to be approximately $250 \mathrm{~nm}$. Subsequent tests were configured to ensure that some particles in this size range where introduced to the graphite surface during penetration tests.

- It proved to be challenging to generate $\mathrm{UO}_{2}$ by oxidizing uranium metal in an environment prototypic of a graphite reactor so an existing Redding $\mathrm{EU} \mathrm{UO}_{2}$ source was used for penetration tests. The Redding material contained $250 \mathrm{ppm}$ of $\mathrm{U}_{236}$ that was characteristic of irradiated uranium and would not be present as an impurity in the PGX graphite. Prior to using the EU in aerosol tests, the material was processed to ensure that some particles in the 1 to $0.1 \mu \mathrm{m}$ range were present.

- An aerosol transport system (ATS) was designed to conduct these studies. A prototype, nonradioactive system was built and tested with $\mathrm{Fe}_{2} \mathrm{O}_{3}$ and WC surrogate materials. Three stage filter tests with the ATS showed that measurable quantities of particles in the size range of $\sim 0.1$ to $\sim 5$ microns could consistently be delivered to the surface of a graphite sample. These tests also showed that a 5 micron pre-filter was needed to prevent swamping the surface of the graphite sample with a large mass of particles greater than 5 microns.

- A radioactive ATS was built and used to conduct one $\mathrm{UO}_{2}$ three stage filter test and three graphite penetration tests. The results of the three stage filter test were comparable to the surrogate tests and indicated that $\sim 2.7 \mu \mathrm{g}$ of particles in the $\sim 0.1$ to $\sim 5$ micron range would be supplied to the surface of the graphite sample in each of the three penetration tests.

- The radioactive ATS graphite penetration tests showed that $\mathrm{UO}_{2}$ particles could penetrate $35 \mathrm{~mm}$ into a PGX graphite sample. $\mathrm{UO}_{2}$ concentrations as high as $10 \mathrm{ppb}$ (approximately equal to the expected background levels in reactor graphite) were measured at depths as great as $15 \mathrm{~mm}$. 
- The representative collector size (i.e., filtration length scale) for the unit collector model was adjusted to fit the $\mathrm{UO}_{2}$ penetration results from the radioactive ATS graphite penetration tests. The collector size determined from the fitted data was more than twice as large as the preliminary estimates that were made based on the permeability and assumed representative pore size. The higher collector size indicted from the empirical data significantly changed the model and lead to predictions of dramatically deeper particle penetration and a somewhat larger maximally penetrating particle diameter of about $350 \mathrm{~nm}$.

- Graphite penetration tests indicate that it is possible, under optimum conditions, for uranium from fuel failures to be transported by the carbon dioxide gas coolant system and deposited at depths as great as $35 \mathrm{~mm}$ in the graphite blocks of a graphite moderated reactor. However, the penetration levels of uranium seen under optimum may not be realized when the $\mathrm{UO}_{2}$ is generated from fuel failures in an actual reactor environment. Nonetheless, these tests show that steps must be taken to identify and ensure that uranium contamination from fuel failures does not adversely impact a GIRM assessment.

- A standard protocol to evaluate whether a GIRM graphite sample has been compromised by uranium from fuel failures should be implemented and appropriate steps should be taken to ensure that any compromised sample uses other indicator elements for GIRM assessment. 


\subsection{Acknowledgements}

The authors would like to acknowledge the efforts of many staff that supported this activity. Specifically, we appreciated Chris Brown and Cristian Iovin's assistance with ICP-MS measurements and analysis. Calvin Delegard provided valuable insight regarding $\mathrm{UO}_{2}$ selection, assisting with uranium oxidation testing and general project direction. We greatly appreciated the efforts of Alla Zelenyuk who provided filter efficiency equipment for use and offered her assistance, as well as her post-doctoral staff. The authors also thank Mac Zumhoff for his tireless efforts in operating the aerosol transport system and Anne Kozelisky for operating the radiological aerosol transport system. We also thank Brian Riley and Carolyne Burns for assisting with SEM imaging and PSD measurements. 

PNNL-21014

\subsection{References}

Dullien, F.A.L. Porous Media: Fluid Transport and Pore Structure. 1992.

Le'on y Le'on, Carlos A. New Perspectives in Mercury Porosimetry. Advances in Colloid and Interface Science. 1998. p. 341-372.

Elimelech, M., Particle deposition and aggregation : measurement, modelling, and simulation. Colloid and Surface Engineering Series. 1995, Oxford [England]: Boston. xv. p. 441.

Friedlander, S. K. (2000) Smoke, dust, and haze : fundamentals of aerosol dynamics, New York ; Oxford, Oxford University Press.

Geankoplis, C. J. (1993) Transport processes and unit operations, Engelwood Cliffs, N.J., PTR Prentice Hall.

Otani, Y., C. Kanaoka, and H. Emi. Experimental-Study Of Aerosol Filtration By The Granular Bed Over A Wide-Range Of Reynolds-Numbers. Aerosol Science And Technology. 1989. 10(3), p. 463-474.

Konstandopoulos, A.G., E. Skaperdas, and M. Masoudi. Microstructural Properties of Soot Deposits in Diesel Particulate Traps. SAE. 2002. 2002-01-1015.

Miyairi, Y., T. Noguchi, T. Hiramatsu, S. Hirose, M. Ogawa, S. Miwa, and P. Busch. Diesel Particulate Filter (DPF) Trapping Efficiency Improvement Under No-Soot-Layer Conditions Obtained Through Pore Size Distribution Optimization. SAE, 2006. 2006-01-1528.

Heikkinen, M.S.A. and N.H. Harley. Experimental investigation of sintered porous metal filters. $J$. Aerosol Science. 2000. 31(6), p. 721-738.

Pacific Northwest National Laboratory (PNNL). 2008. Inductively Coupled Plasma Mass Spectrometric (ICP-MS) Analysis. PNNL-AGG-415, Rev. 2. Pacific Northwest National Laboratory, Richland, Washington.

Pacific Northwest National Laboratory (PNNL). 2008. Inductively Coupled Plasma-Optical Emission Spectrometry (ICP-OES) Analysis. PNNL-AGG-ICP-AES, Rev. 2. Pacific Northwest National Laboratory, Richland, Washington.

Pacific Northwest National Laboratory (PNNL). 2009. Environmental Sciences Laboratory Quality Assurance Plan. ESL-QAP, Rev. 3. Pacific Northwest National Laboratory, Richland, Washington.

C. J. Gesh, "A Graphite Isotope Ratio Method Primer - A method for Estimating Plutonium Production in a Graphite Moderated Reactor", PNNL-14568, Pacific Northwest National Laboratory, February 2004. 


\section{Appendix A - Porosity and Permeability Review}

The graphite used in nuclear reactors is a porous material. A porous media is capable of sustaining fluid flow through the internal pores (i.e., channels) that make up the material's structure. Porosity $\varphi$ is the fraction of void space to the bulk volume of the porous sample and therefore defines how much space the pores occupy (Dullien, 1992). Porosity is expressed as

$$
\varphi=1-\frac{\rho_{\text {bulk }}}{\rho_{g}}
$$

where $\rho_{\text {bulk }}$ is the graphite bulk density and $\rho_{g}$ is the graphite material density. The bulk density is the measured mass to volume ratio, whereas the material density is the theoretical density of a graphite material.

Permeability is a measure of the ability of fluids to flow through porous media. It is controlled by the pore structure of the material and governed by Darcy's Law. One-dimensional Darcy's Law is given by

$$
Q_{2}=\frac{k A}{\mu} \frac{\Delta P}{L}
$$

where $Q_{2}$ is the volumetric flow rate at the exit face, $k$ is the intrinsic permeability of the material, $A$ is the cross-sectional area, $\mu$ is the dynamic viscosity of the fluid, $\Delta P$ and $L$ are the pressure drop and length of the porous material, respectively. Typically, permeability is measured in darcy's, where 1 darcy is equal to $0.987 \mu \mathrm{m}^{2}$. This form of Darcy's Law is valid for one-dimensional laminar incompressible linear flow and provides the relationship between the permeability and ratio of flow rate and pressure drop.

When the flowing fluid is compressible, Darcy's Law must be corrected to account for the effects of gas compression. Including gas compression, Darcy's Law becomes

$$
Q_{2}=\frac{k A}{\mu} \frac{\bar{P}}{P_{2}} \frac{\Delta P}{L}
$$

where $\bar{P}$ is the average of the inlet and exit pressure and $P_{2}$ is the exit pressure.

The permeability is determined from the ratio of the pressure and flow. A simple flow system was developed to apply a known pressure drop and a measureable resultant flow rate. The pressure drop across a graphite block in a typical reactor under normal operating conditions is 10 psig; therefore, a 10 psig pressure drop was selected as the highest pressure drop used in all permeability and aerosol flow experiments. Five pressure drop values were chosen: 1, 2, 5, 7.5 and $10 \mathrm{psig}$, to ensure an accurate permeability measurement. Due to the inhomogeneous nature of graphite, individual measurements of all graphite samples used in these experiments were conducted. 
The pressure-driven flow system consisted of a pressurized gas tank (i.e., nitrogen, $\mathrm{CO}_{2}$, etc), fitted with an isolation valve and pressure regulator. The regulator controlled the pressure to a tee, where one outlet was connected to a digital pressure gauge and the other to a ball valve. The potted sample was connected, via tubing, to the ball valve, which allowed the flow to be isolated while changing samples. The gas flow rate was measured using a bubble flow meter, which was connected to the outlet of the potted sample. A schematic diagram of the flow system is shown in Figure 31.

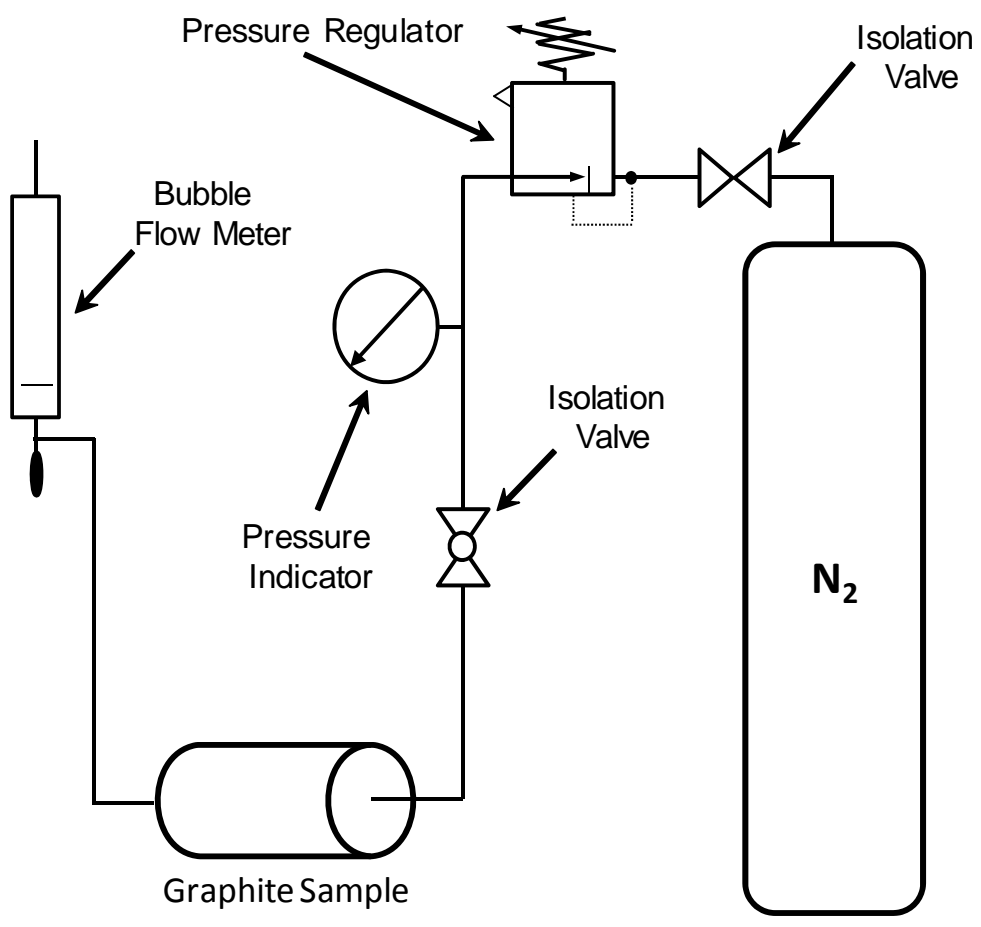

Figure 31. Permeability experimental setup. 


\section{Appendix B - Graphite SEM Images}

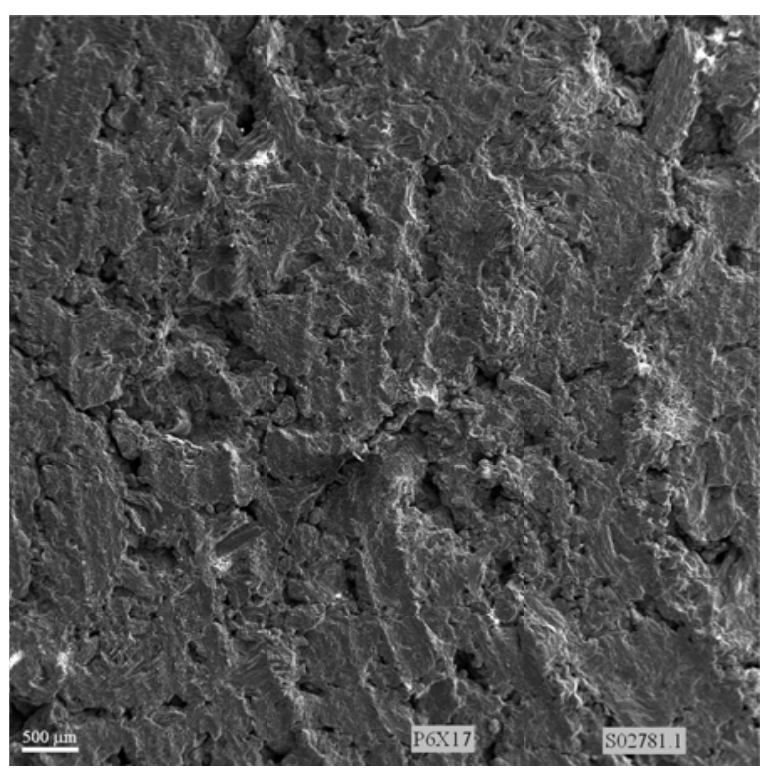

(a) Left of center

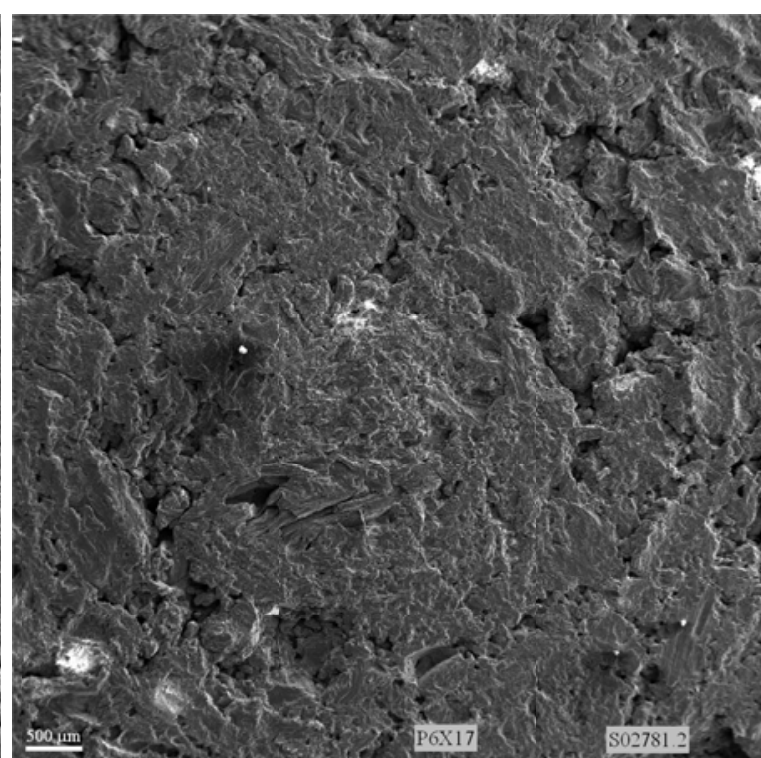

(b) Center

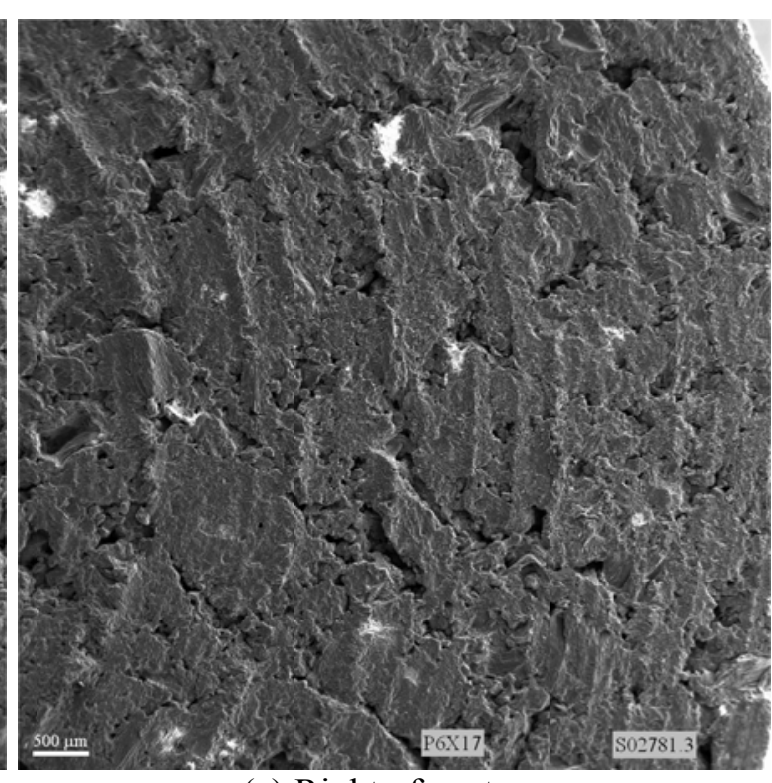

(c) Right of center

Figure 32: SEM images of PGX sample 17 inlet cross-section at 12x magnification. The machining "rings" are clearly identifiable in (a) and (c). 


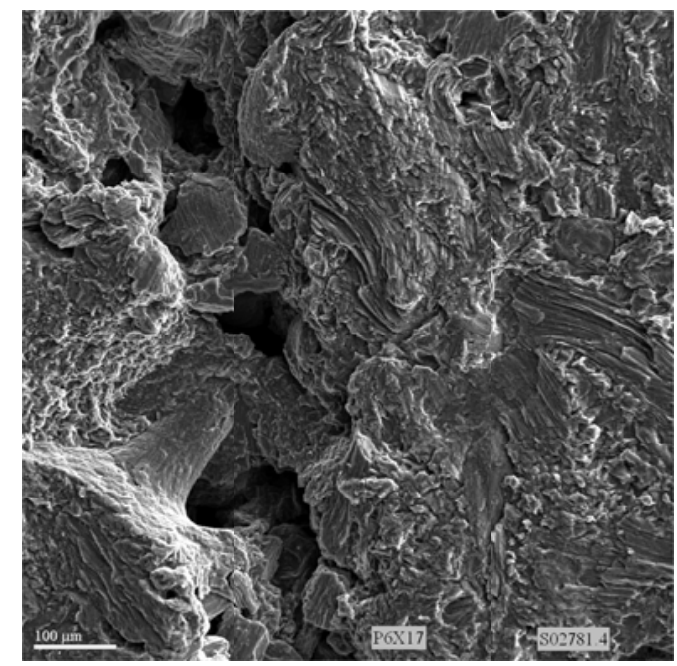

(a)

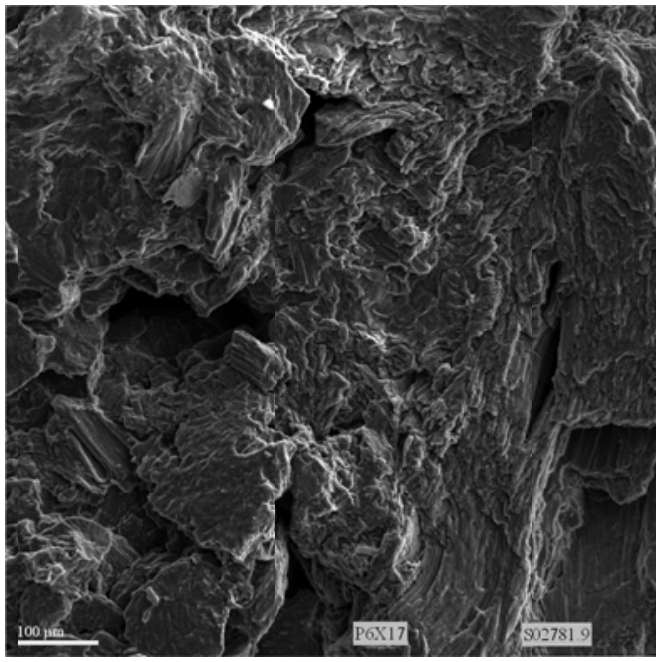

(d)

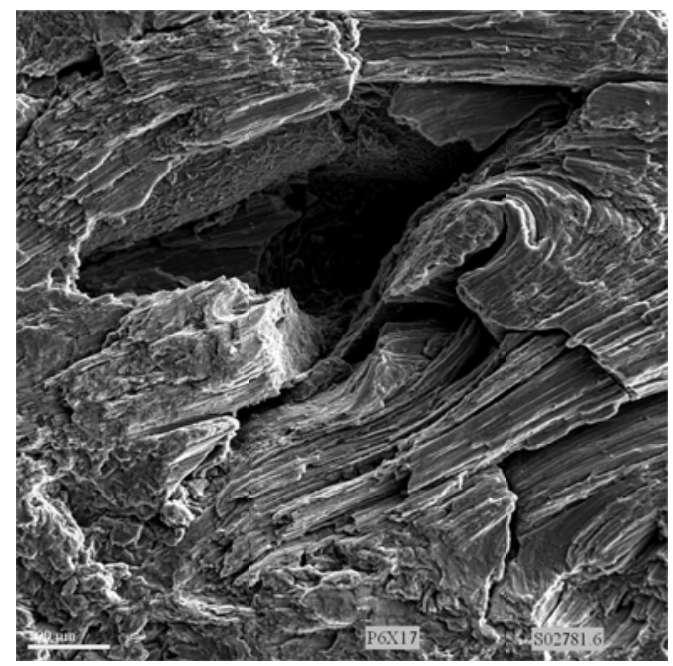

(b)

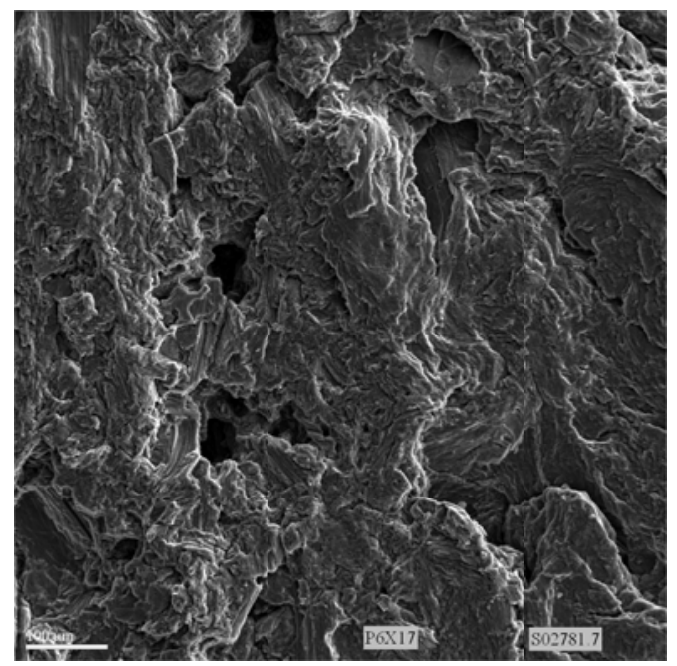

(c)

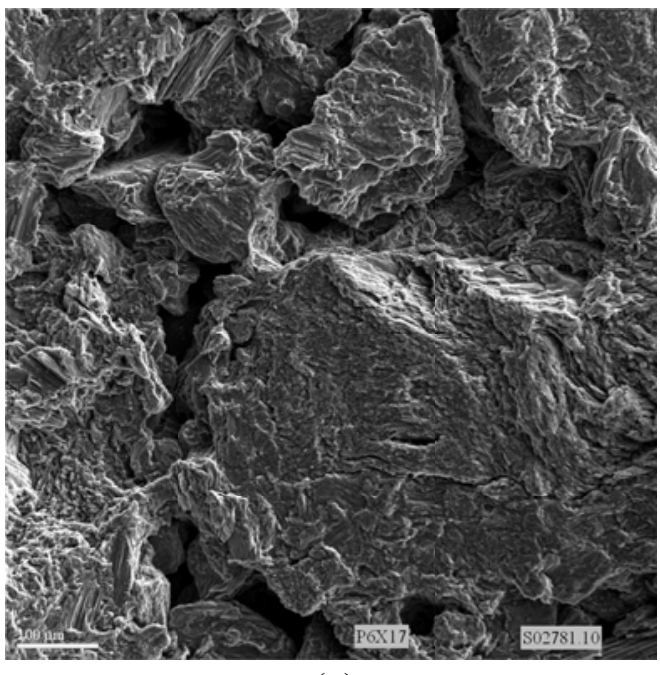

(e)

Figure 33: SEM images of PGX sample 17 inlet cross-section at 100x magnification. Pores ranging from 50 - $100 \mu \mathrm{m}$ can be seen in (a), (c), (d) and (e). A large 150 by $600 \mu \mathrm{m}$ pore is present in (b). 


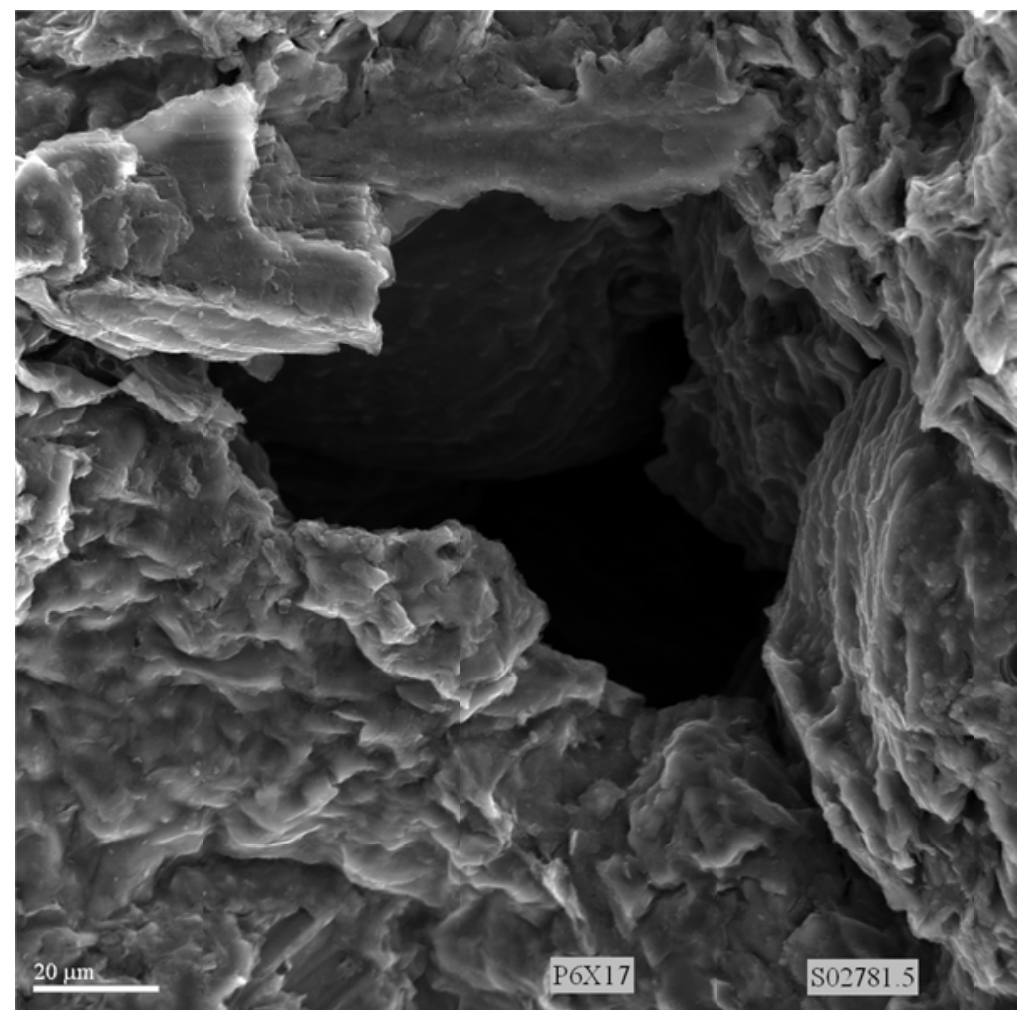

(a)

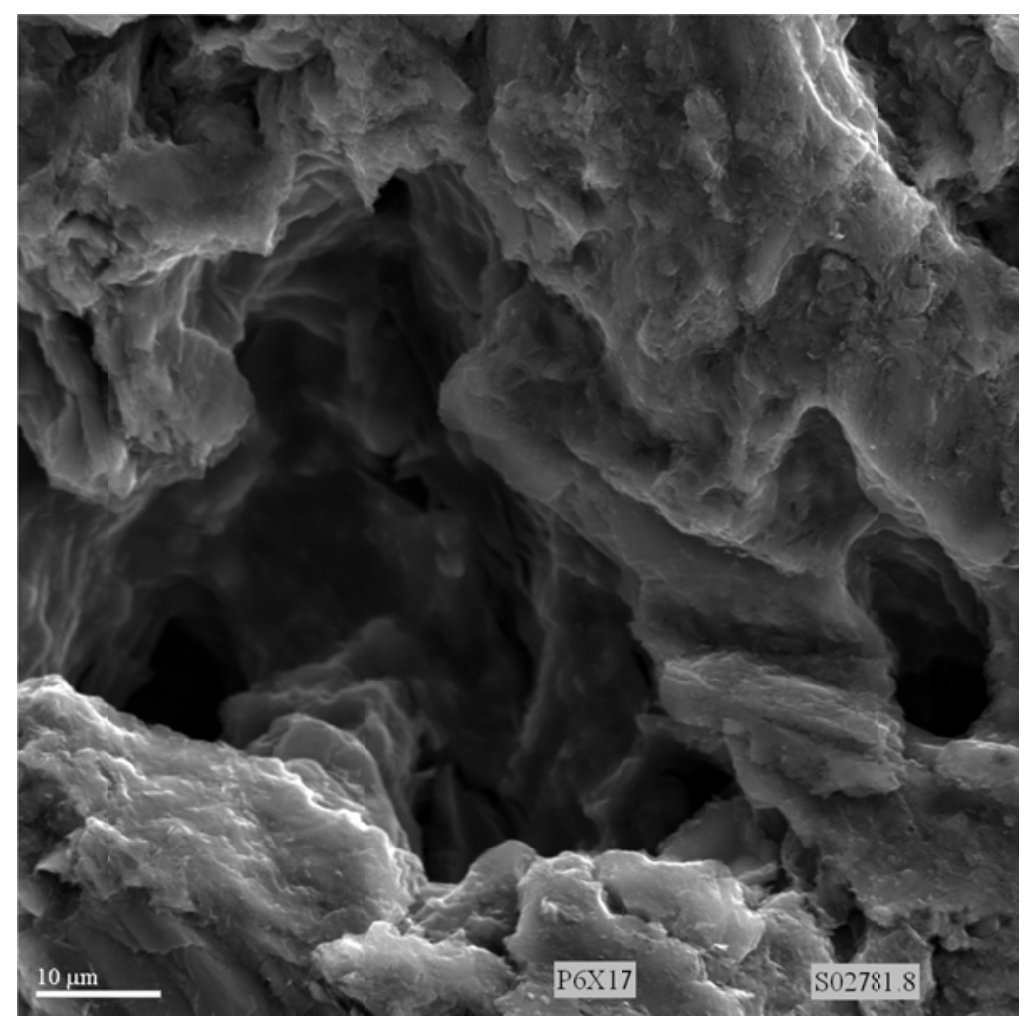

(b)

Figure 34: SEM images of PGX sample 17 inlet cross-section. The magnification of (a) and (b) is 500 and 1000x, respectively. 


\section{Appendix C - Filter Efficiency Experiments}

The filtration properties of the graphite material were desired due to the assumptions associated with the unit collector model. Therefore, filter efficiency experiments (FEE) were proposed as an experimental method to test the graphite filtration properties and validate the unit collector model.

The filter efficiency experimental system consists of subjecting thin graphite slices to a flow of an aerosol with well characterized particles. The experiment is shown graphically in Figure 35 and is comprised of an atomizer, diffusion dryer, differential mobility analyzer (DMA), condensation particle counter (CPC), pressure transducers and mass flow meters. Clean air is passed through the atomizer which generates an aerosol containing liquid droplets of an ammonium sulfate solution. The aerosol is passed through the diffusion dryer to remove the liquid, thereby, forming solid ammonium sulfate particles with a broad PSD. The aerosol stream is then size-selected using the DMA to obtain the desired mono-disperse particle size. The aerosol then flows into the test section and through the graphite sample (i.e., filter). Dual CPC's measure the particle count upstream and downstream of the test section, therefore obtaining the ratio of introduced to captured particles. Mass flow meters and pressure transducers are used to quantify flow properties.

The filter efficiency experimental system is a well-established and proven technique to evaluate particle penetration into porous media. However, this is the first known attempt to evaluate graphite as a filter medium. As such, experimental difficulties were encountered and only a single experiment was performed on a $0.94 \mathrm{~mm}$ thick graphite sample. The filtration and transmission results are shown in Figure 36. See Figures 38-42 for raw inlet and outlet particle concentration data. The data from this first trial experiment would indicate that $400 \mathrm{~nm}$ particles are efficiently filtered but the most penetrating particles are smaller than smallest size tested $(40 \mathrm{~nm})$. These limited results are atypical of porous media behavior. An example of a more typical filtration profile that might be expected for a porous media is shown in Figure 37, where the most penetrating particle is approximately $200 \mathrm{~nm}$.

The present limited data must be viewed with a large degree of skepticism given past experience and theoretical insights indicating that $40 \mathrm{~nm}$ particles should be filtered in porous graphite. These results may be an artifact of the thin graphite sample such that a few large pores allowed the aerosol to penetrate the sample unfiltered. This would not be representative of a thicker graphite sample because the large pores would eventually throat down to small pores or run into dead ends. These limited tests demonstrate the potential value of this type of testing but show that additional tests that include thicker graphite samples need to be evaluated prior to drawing any conclusions from this data. 


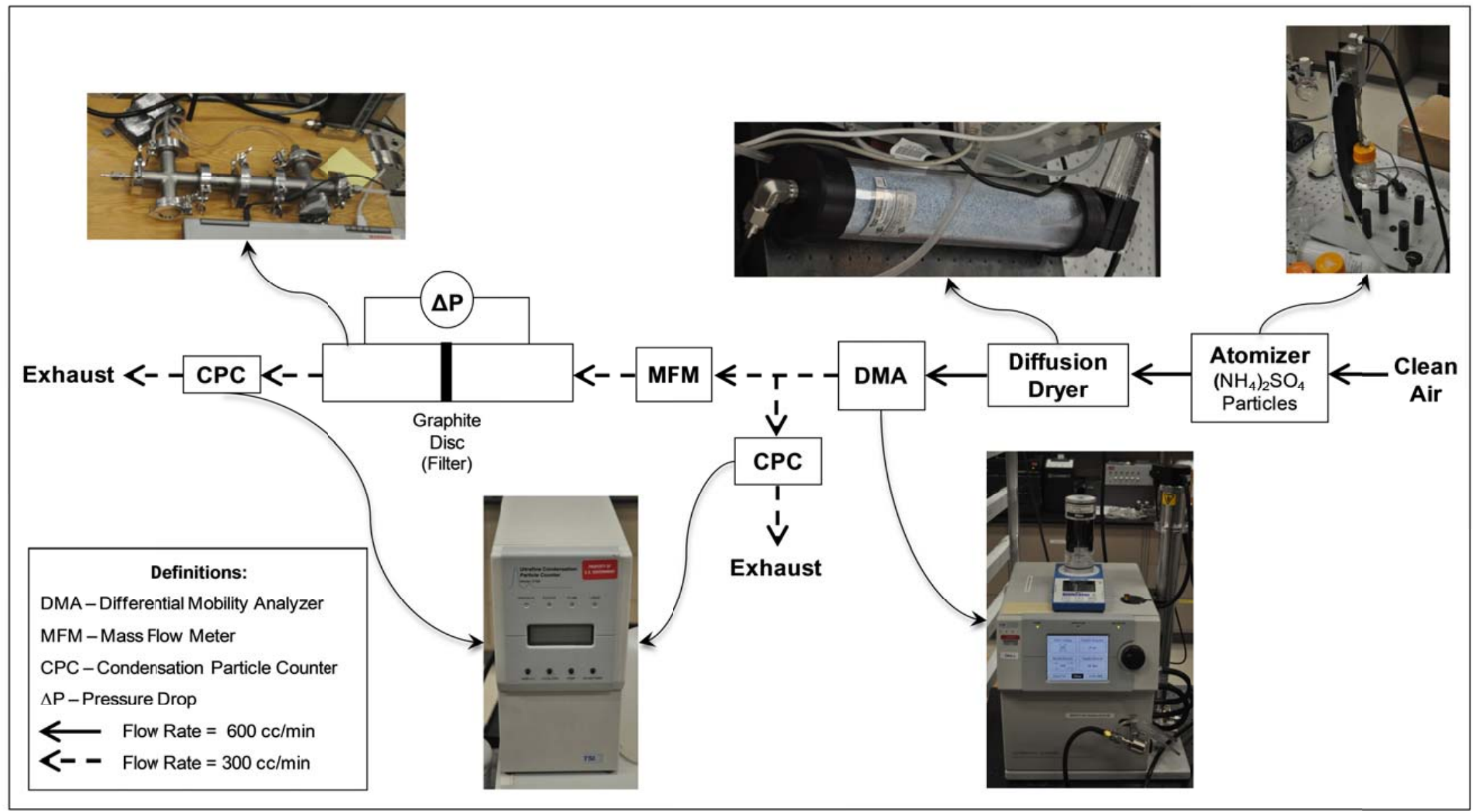

Figure 35: Schematic and photographs of filter efficiency experimental setup. 


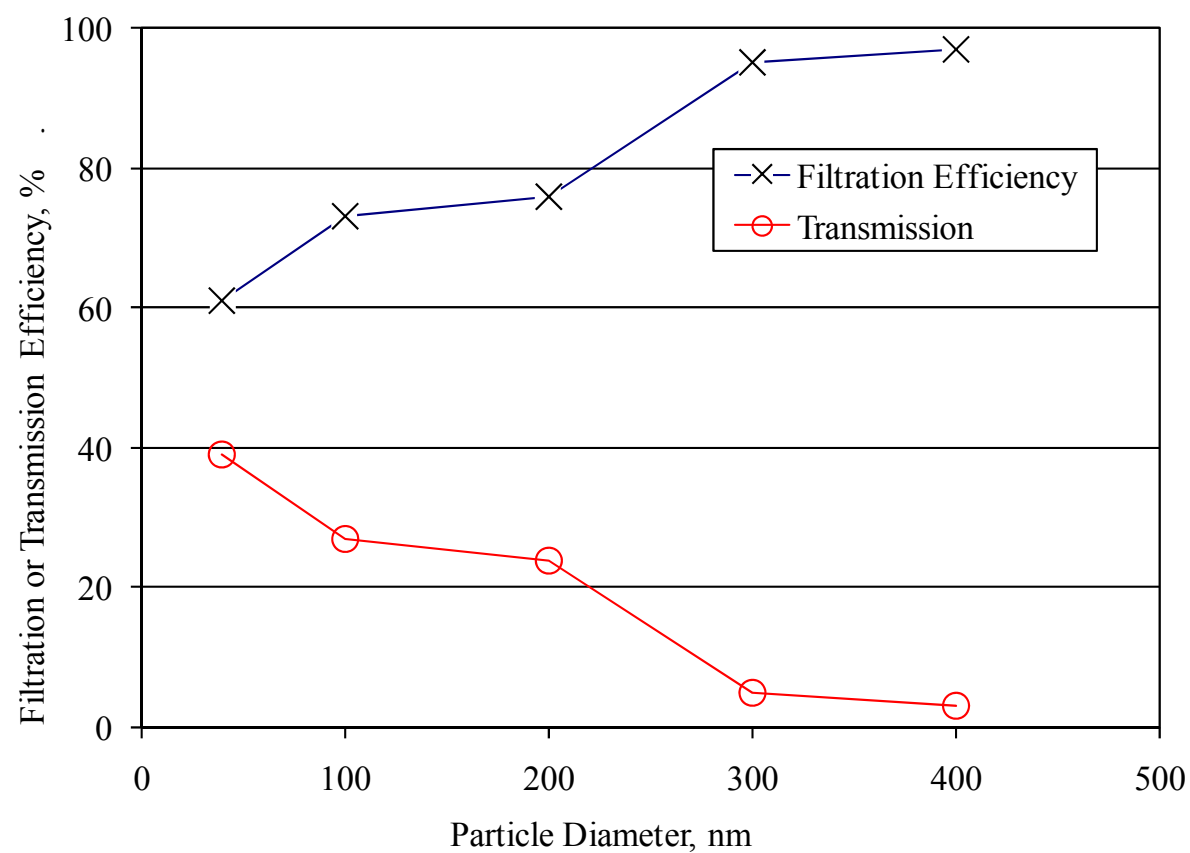

Figure 36: Filter efficiency experiment results for graphite sample FEE21. 


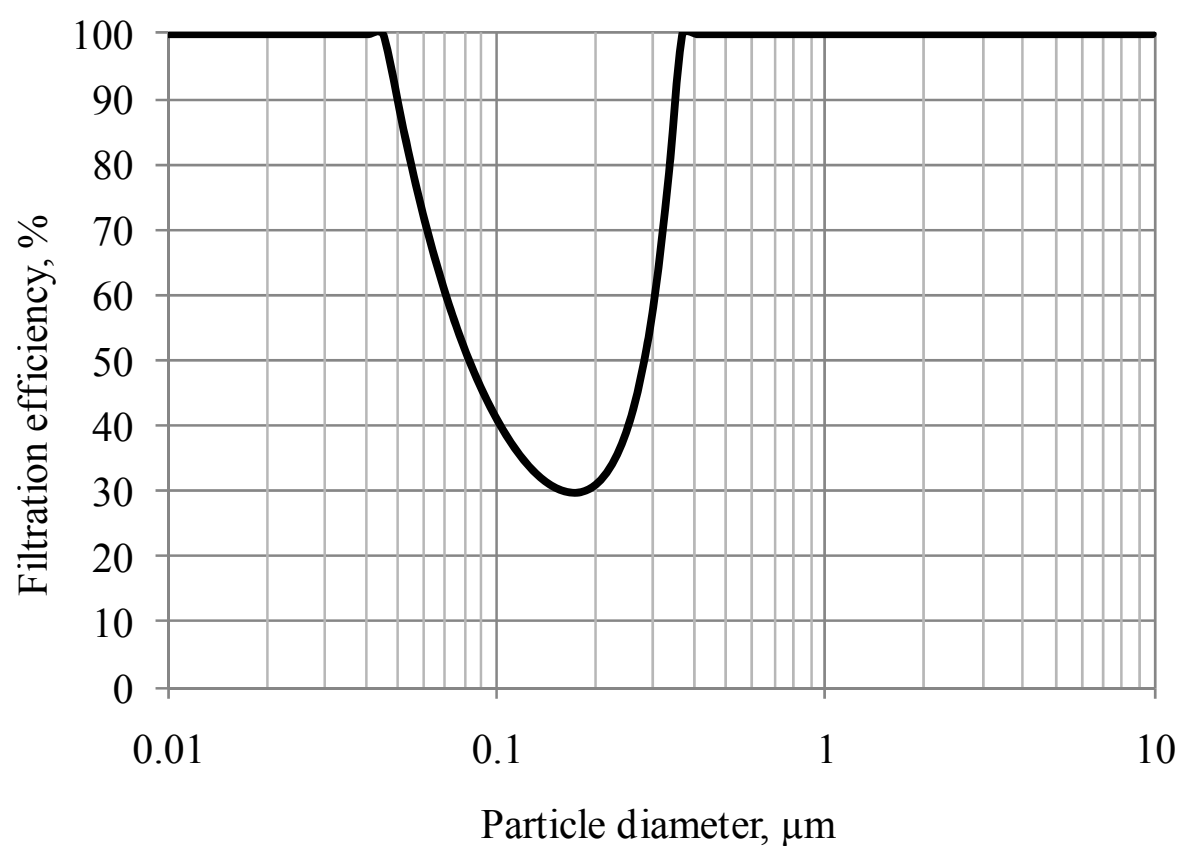

Figure 37: Expected profile of filter efficiency versus particle size. 


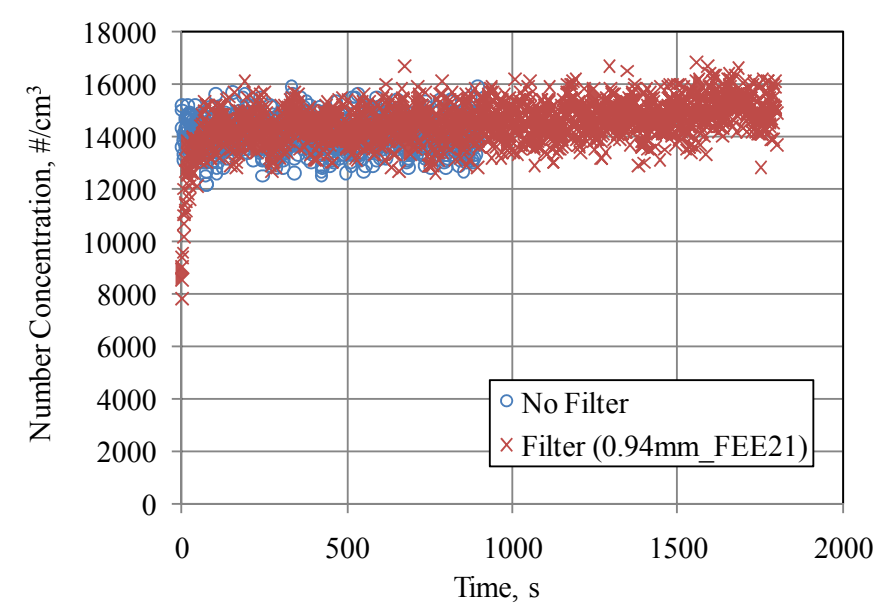

(a) Inlet

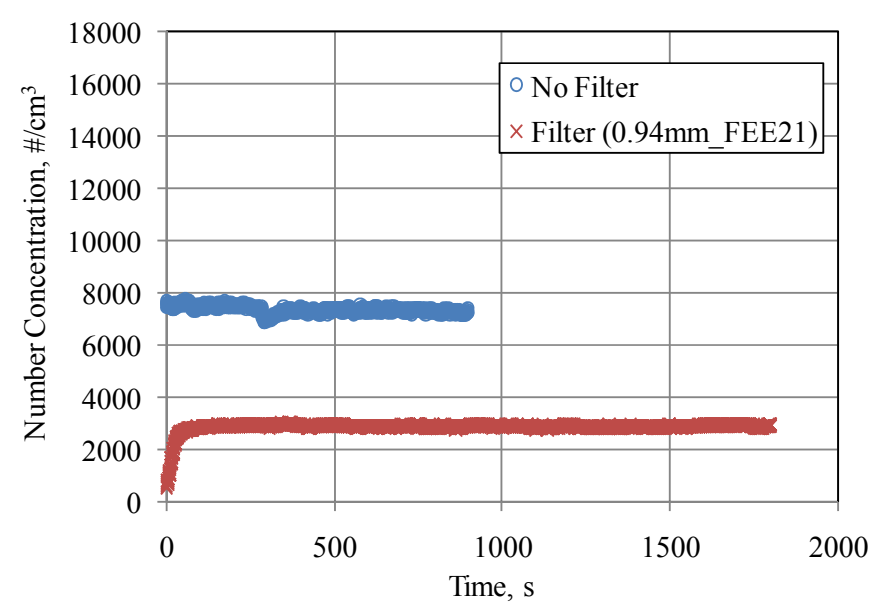

(b) Outlet

Figure 38: Filter efficiency data for $40 \mathrm{~nm}$ particles where (a) and (b) is measured at the inlet and outlet, respectively.

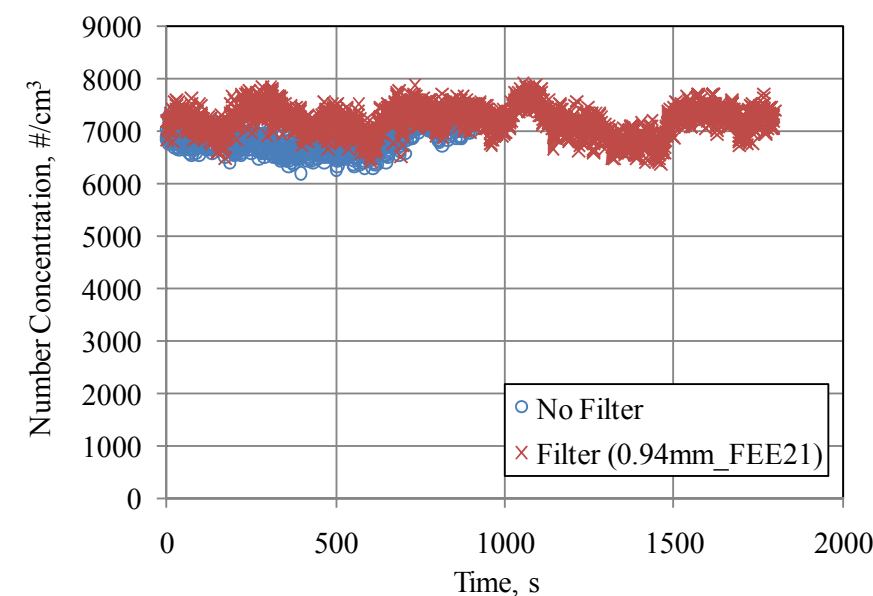

(a) Inlet

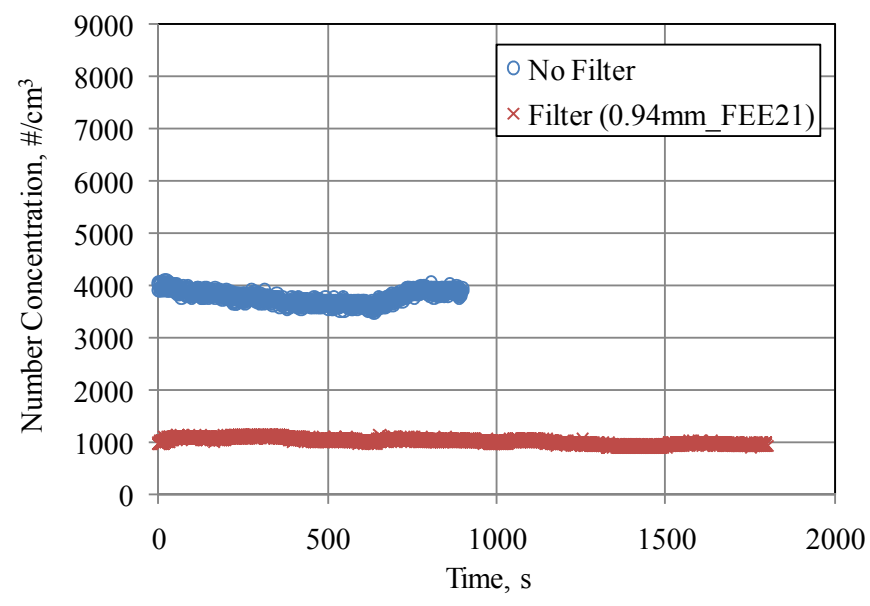

(b) Outlet

Figure 39: Filter efficiency data for $100 \mathrm{~nm}$ particles where (a) and (b) is measured at the inlet and outlet, respectively. 


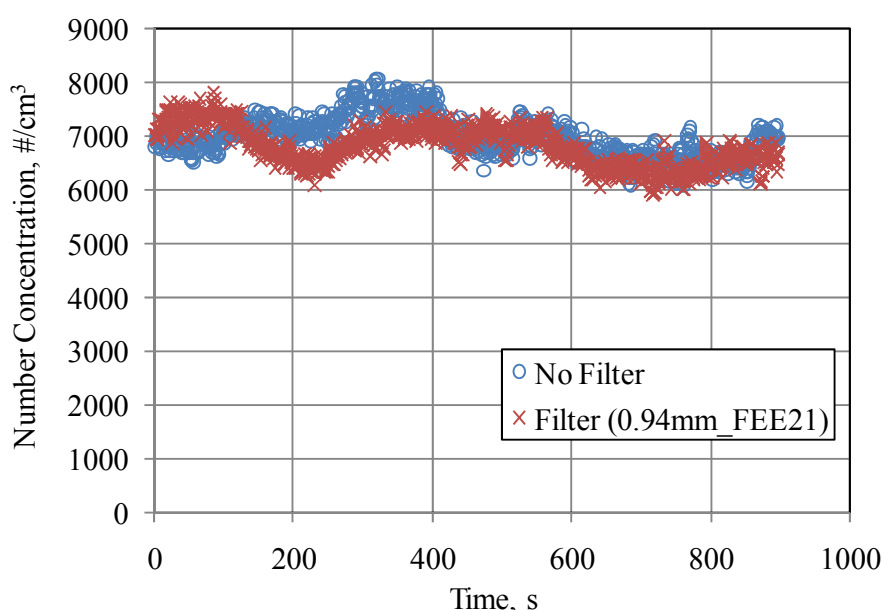

(a) Inlet

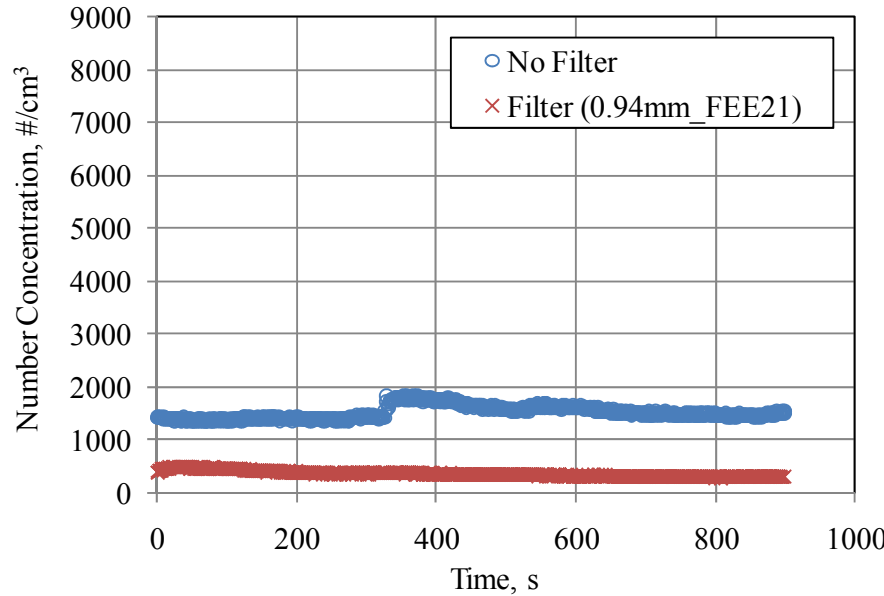

(b) Outlet

Figure 40: Filter efficiency data for $200 \mathrm{~nm}$ particles where (a) and (b) is measured at the inlet and outlet, respectively.

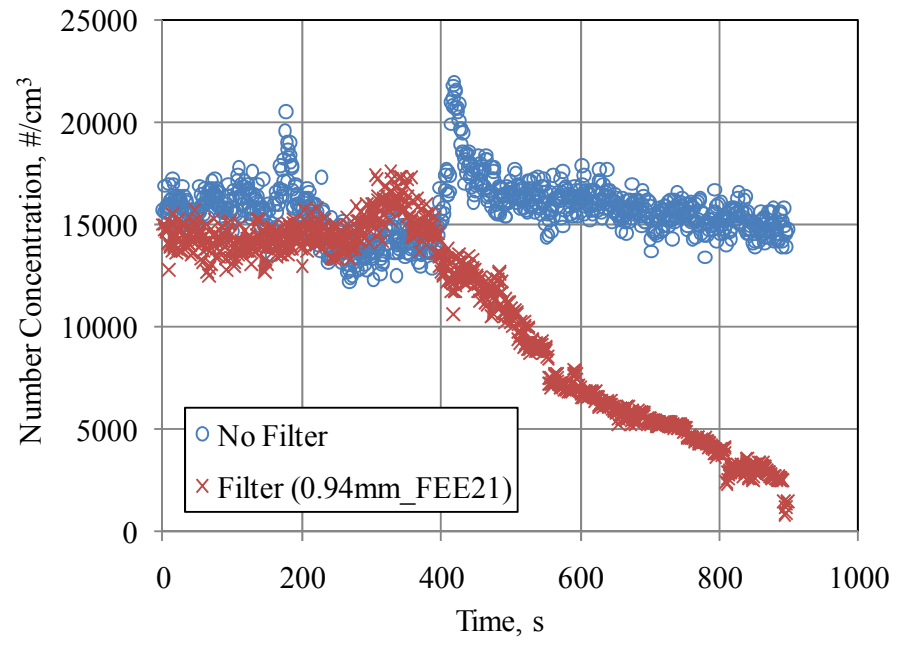

(a) Inlet

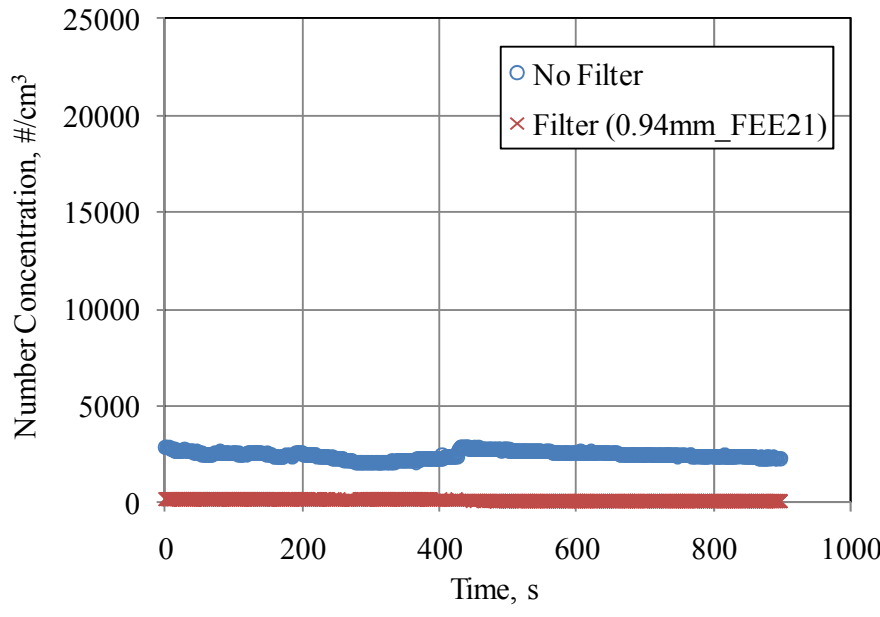

(b) Outlet

Figure 41: Filter efficiency data for $300 \mathrm{~nm}$ particles where (a) and (b) is measured at the inlet and outlet, respectively. 


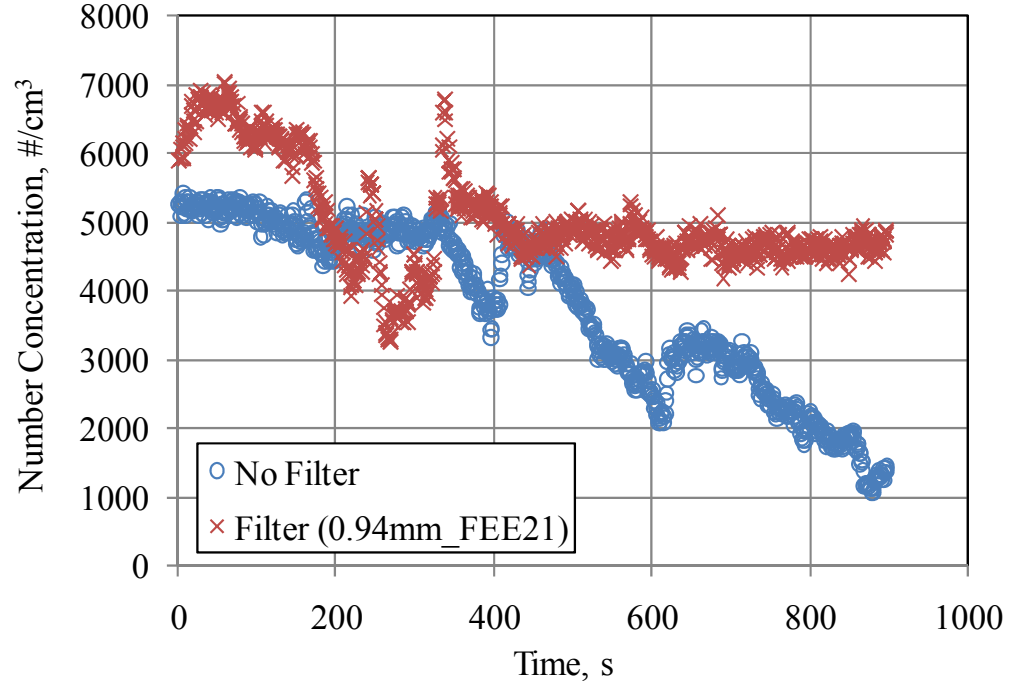

(a) Inlet

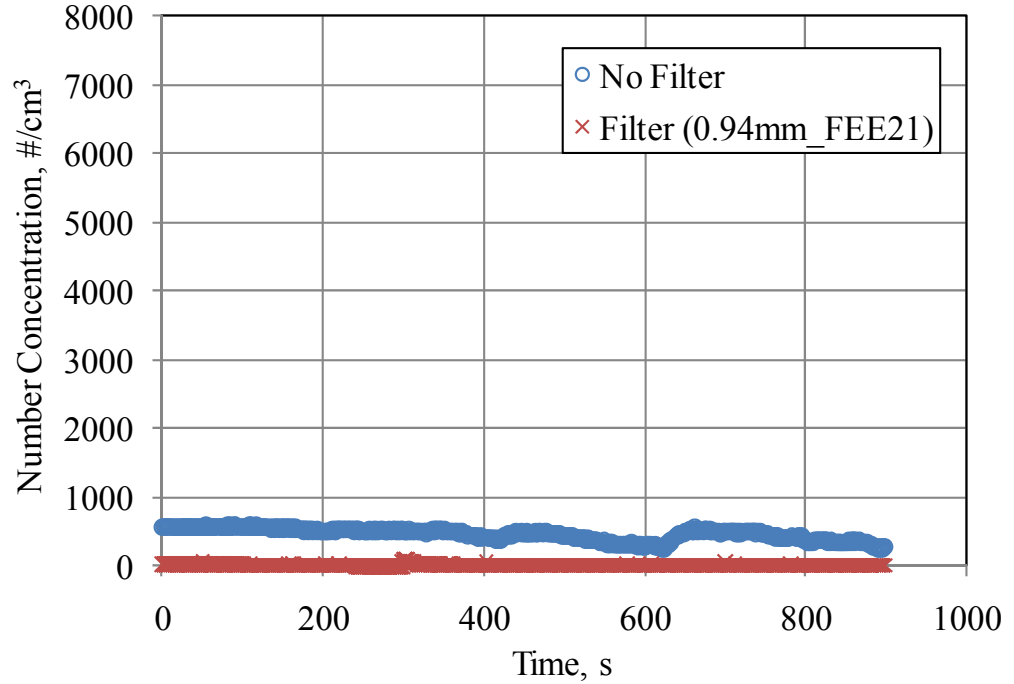

(b) Outlet

Figure 42: Filter efficiency data for $400 \mathrm{~nm}$ particles where (a) and (b) is measured at the inlet and outlet, respectively. 


\section{Appendix D - Aerosol Material SEM Images}

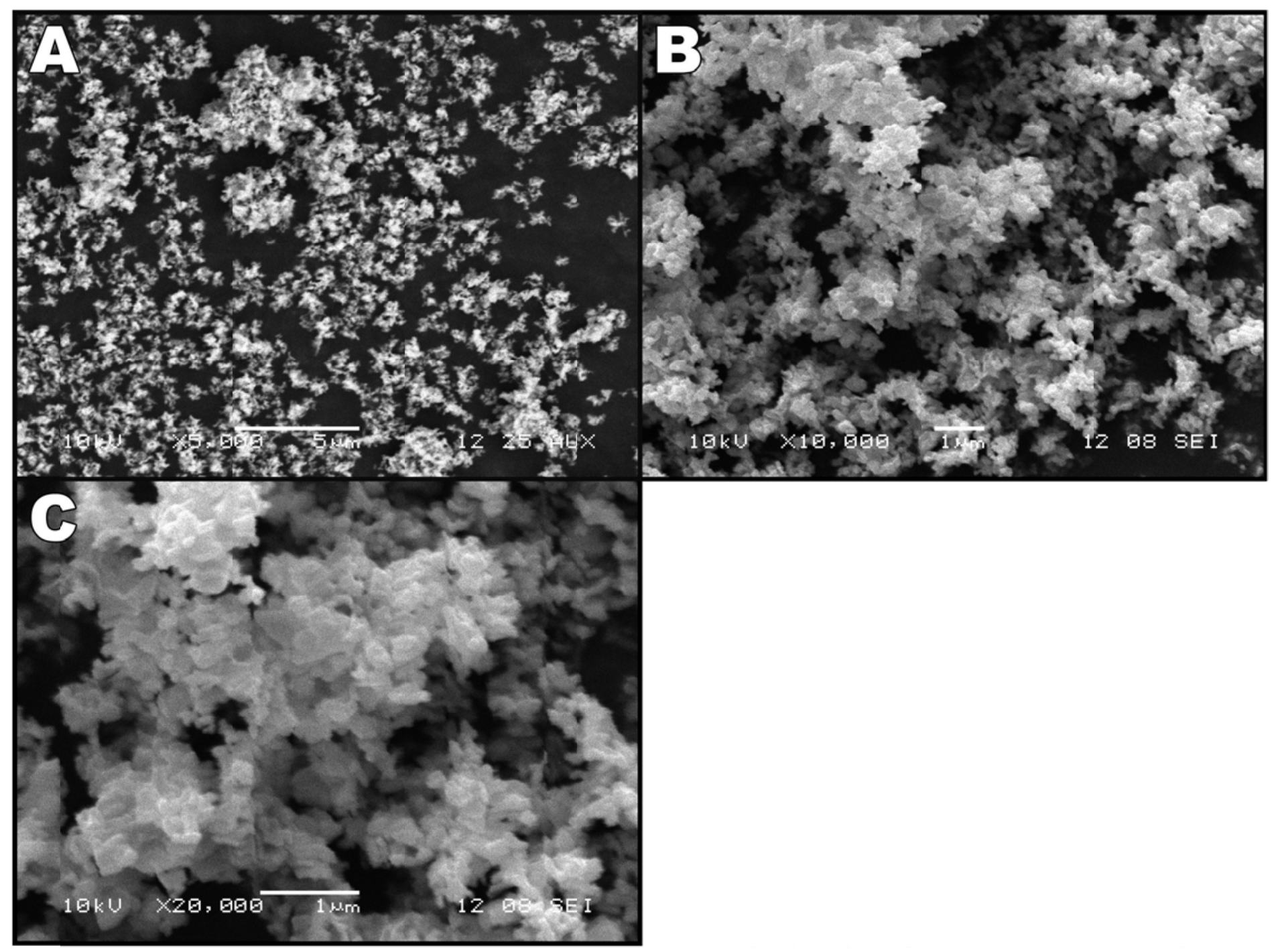

Figure 43: SEM images of iron oxide. Micrographs (A), (B) and (C) were captured at 5,000X, 10,000X and 20,000X, respectively. 


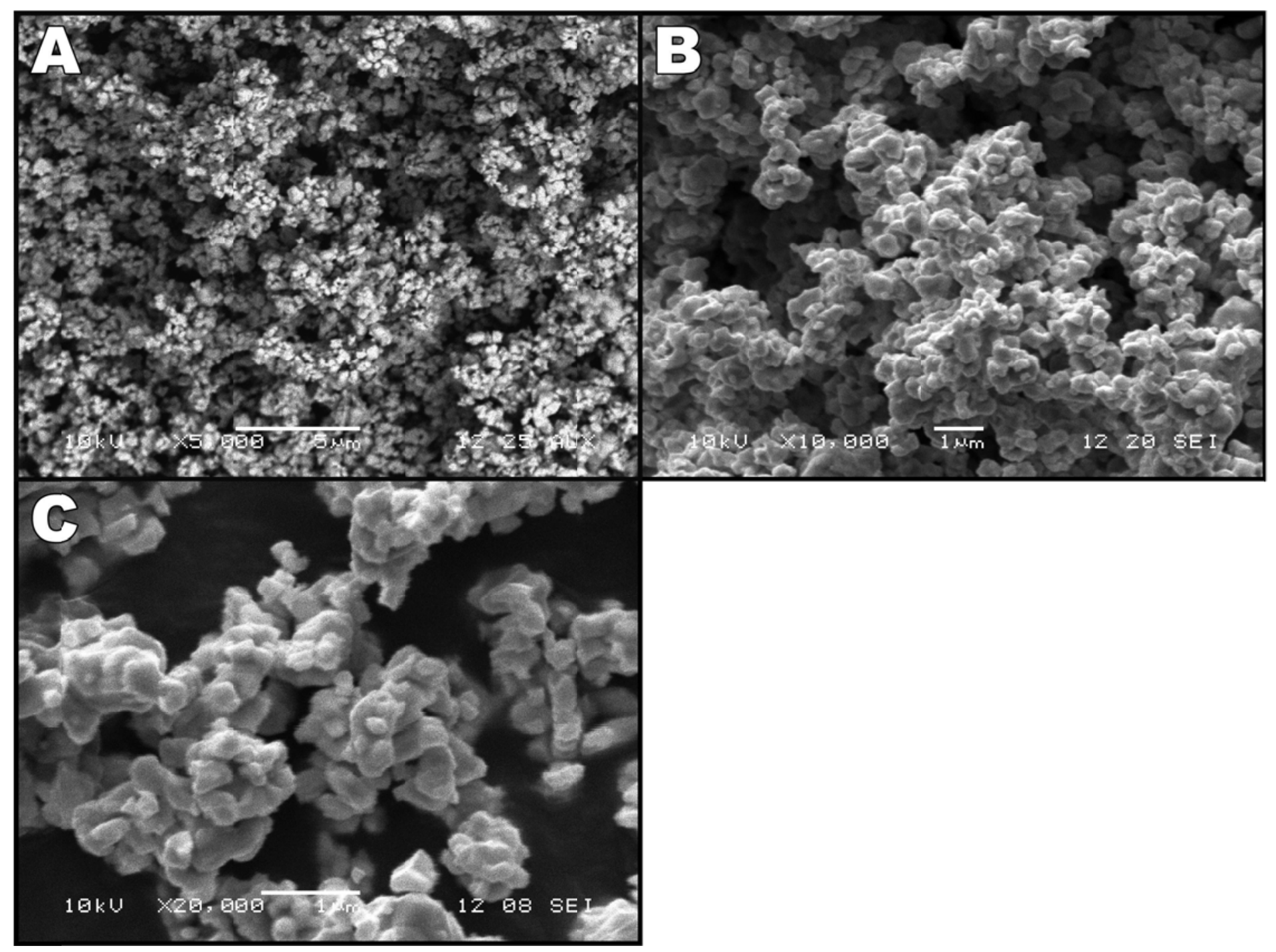

Figure 44: SEM images of tungsten carbide. Micrographs (A), (B) and (C) were captured at 5,000X, 10,000X and 20,000X, respectively. 


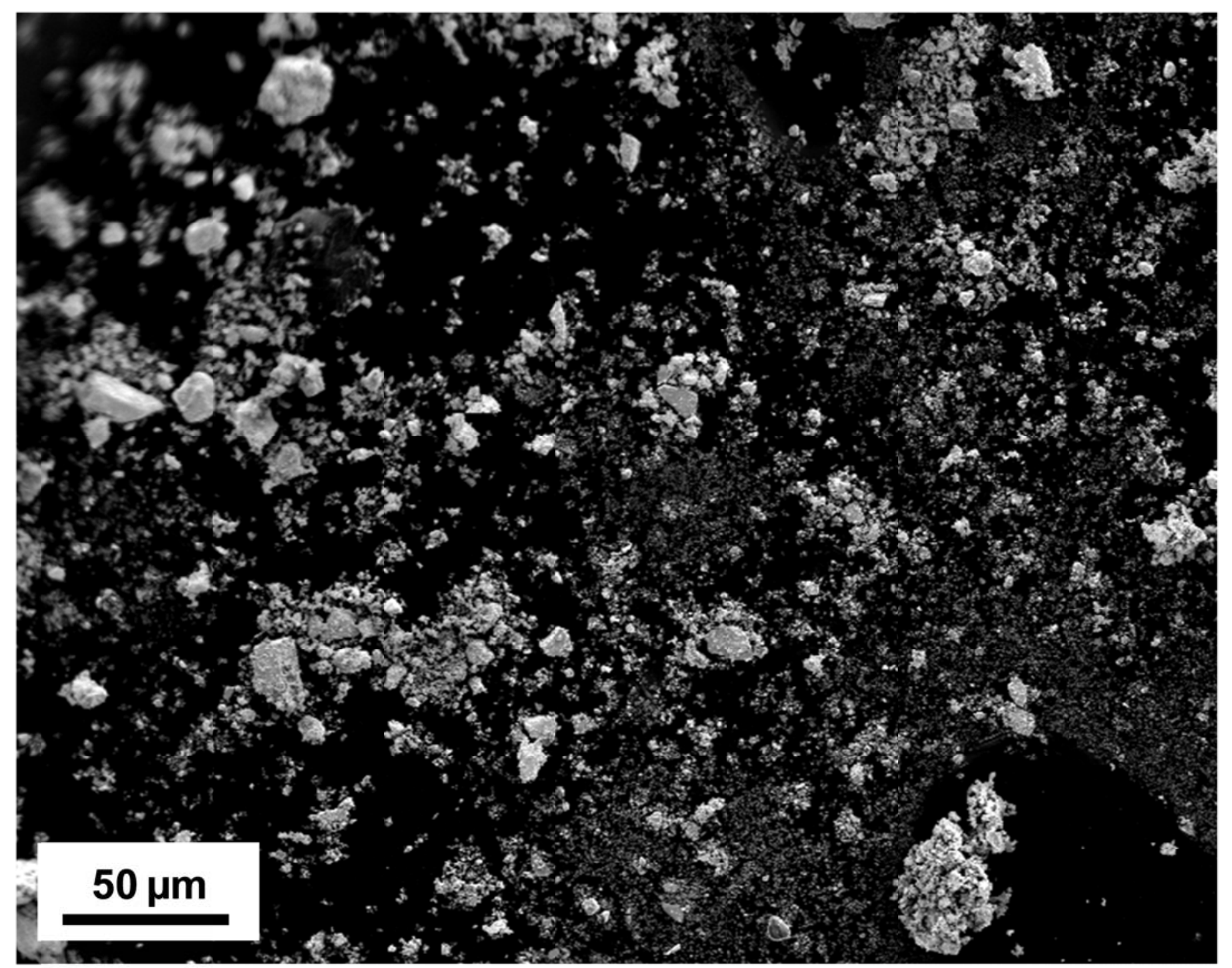

Figure 45: SEM image of uranium oxide. Note many large agglomerates are present. 


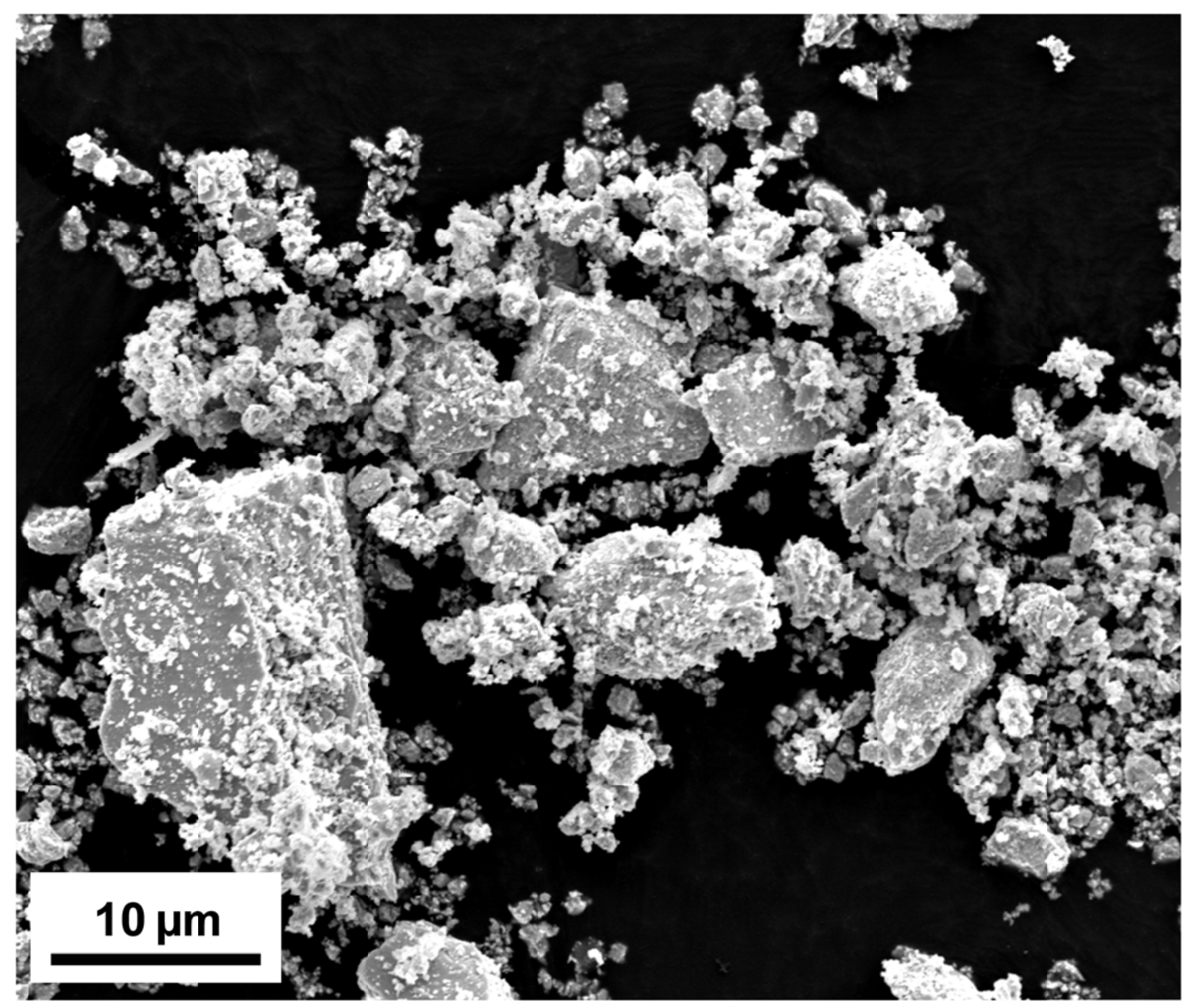

Figure 46: SEM image of uranium oxide showing small order $200 \mathrm{~nm}$ particles affixed to the surface of large order $20 \mu \mathrm{m}$ particles and agglomerates. 


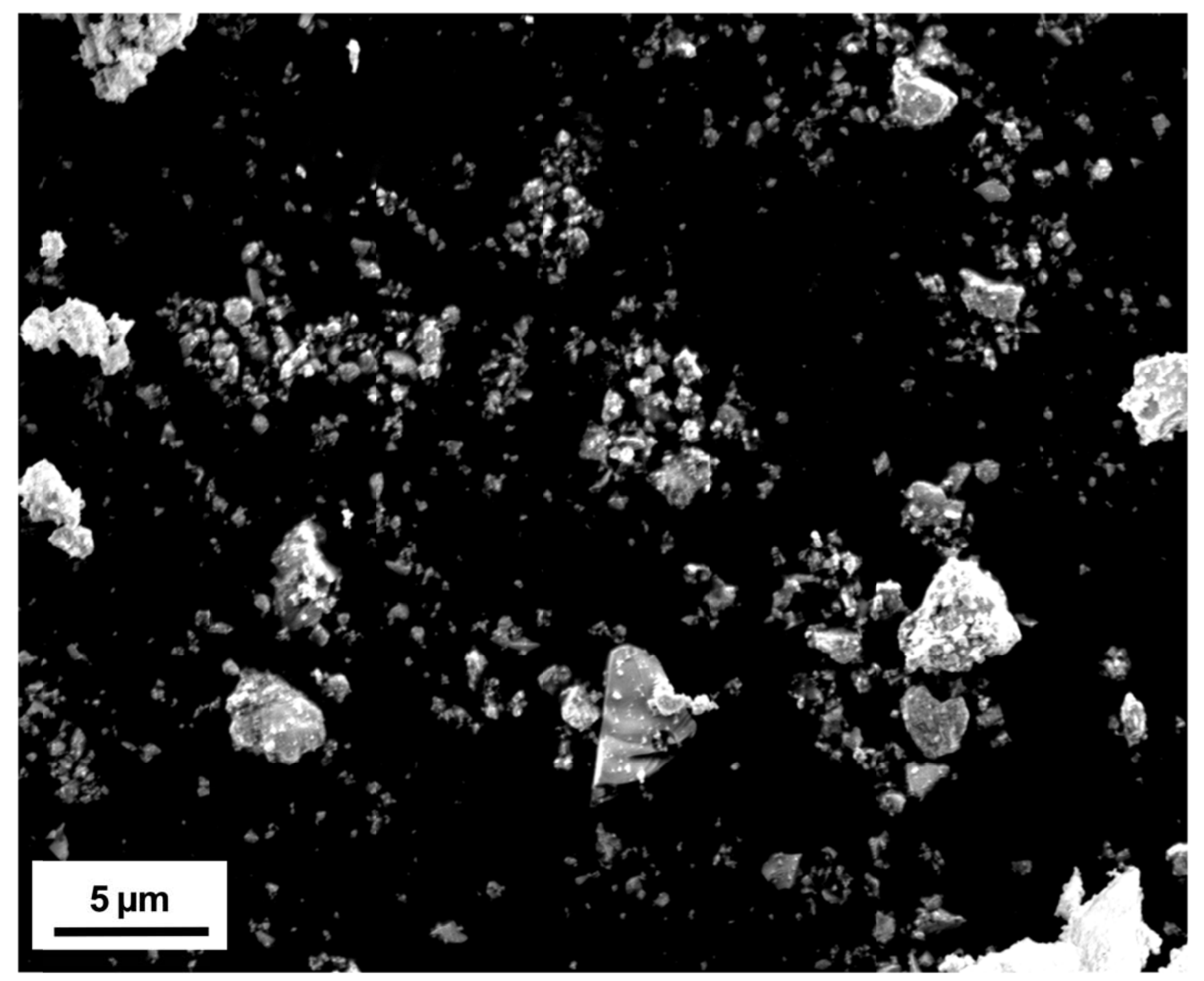

Figure 47: SEM image of uranium oxide showing many small particles. 


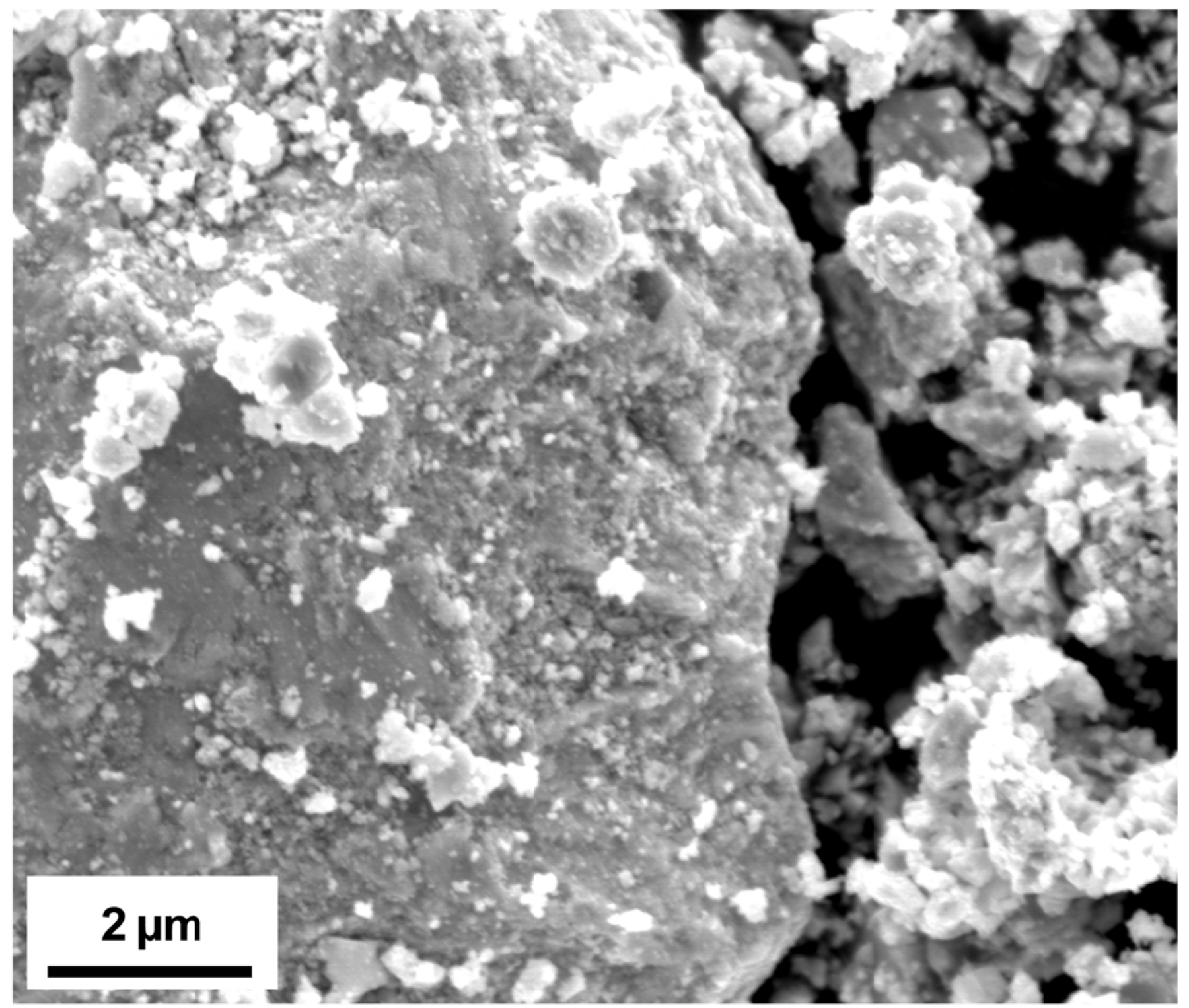

Figure 48: High magnification SEM image of uranium oxide. Extremely small particles can be seen on the surface of large agglomerates. 


\section{Appendix E - Aerosol Transport System Information}

Table 13: ATS 3-stage testing filter specifications.

\begin{tabular}{|c|c|c|}
\hline $\begin{array}{c}\text { Average Filter Pore Size } \\
(\mu \mathrm{m})\end{array}$ & Manufacturer & Model \\
\hline 5 & Fisher Scientific & Fiber paper P5 \\
\hline 2.5 & Whatman & Fiber paper number 5 \\
\hline 0.1 & Millipore & VCTP Isopore membrane filter \\
\hline
\end{tabular}

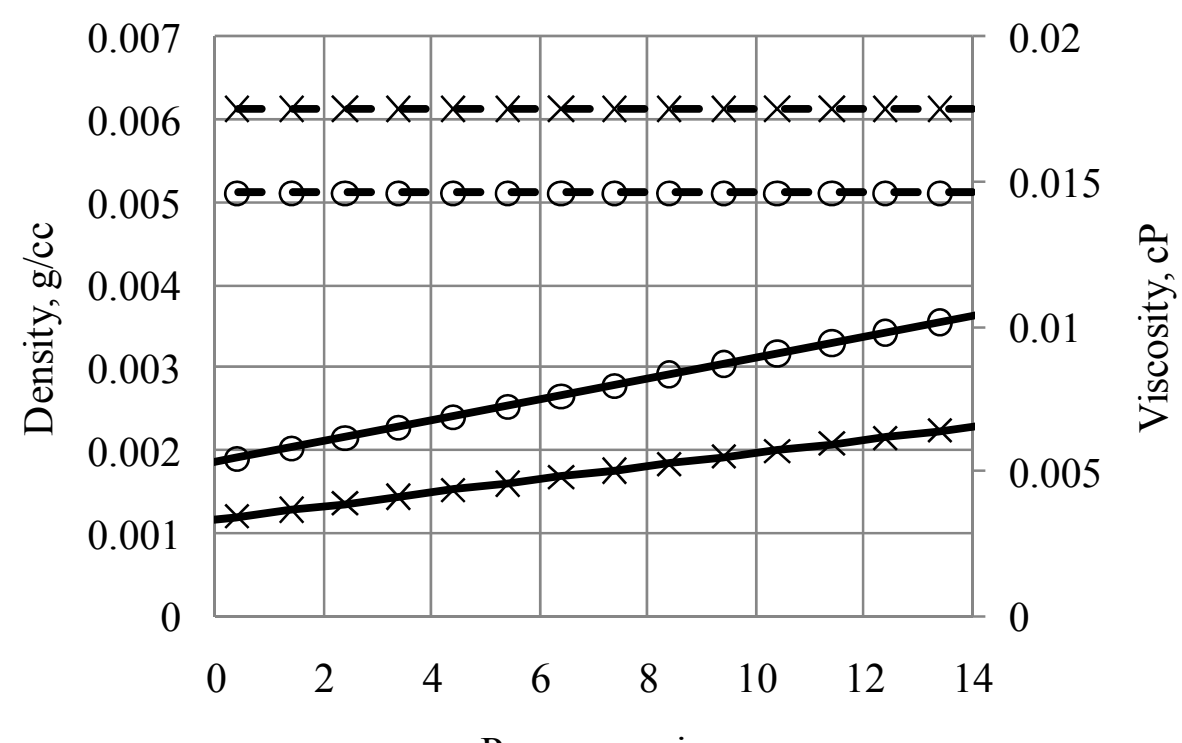

Pressure, psig

× $\mathrm{N}_{2}$ Density $\sim \mathrm{CO}_{2}$ Density $-\times-\mathrm{N}_{2}$ Viscosity $-\mathrm{O}-\mathrm{CO}_{2}$ Viscosity

Figure 49: Comparison of Nitrogen and Carbon Dioxide gas properties. 


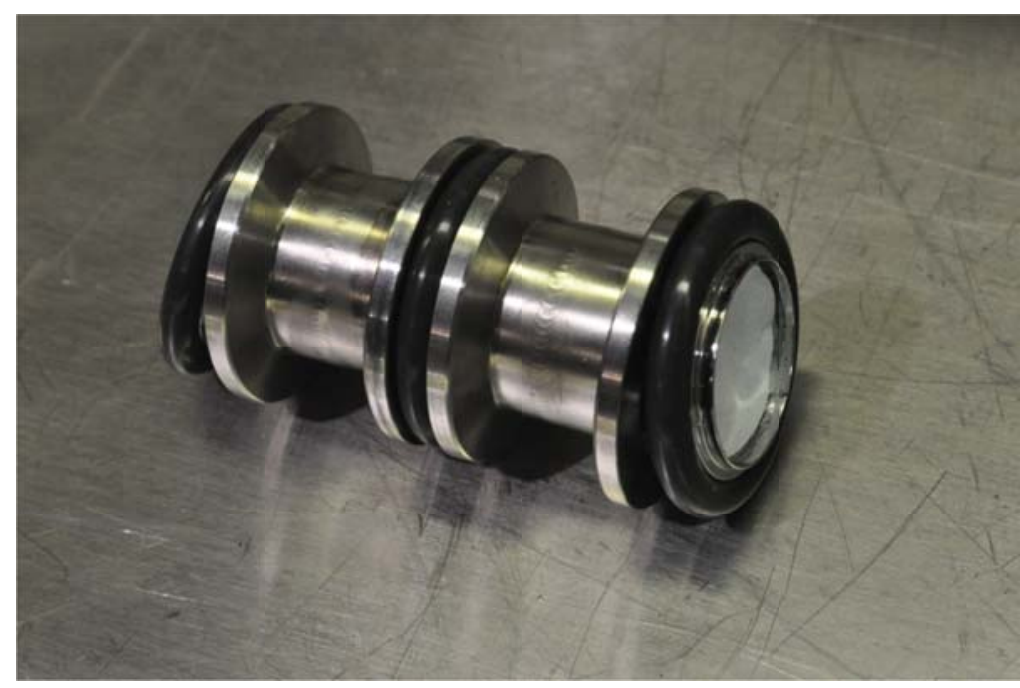

(a)

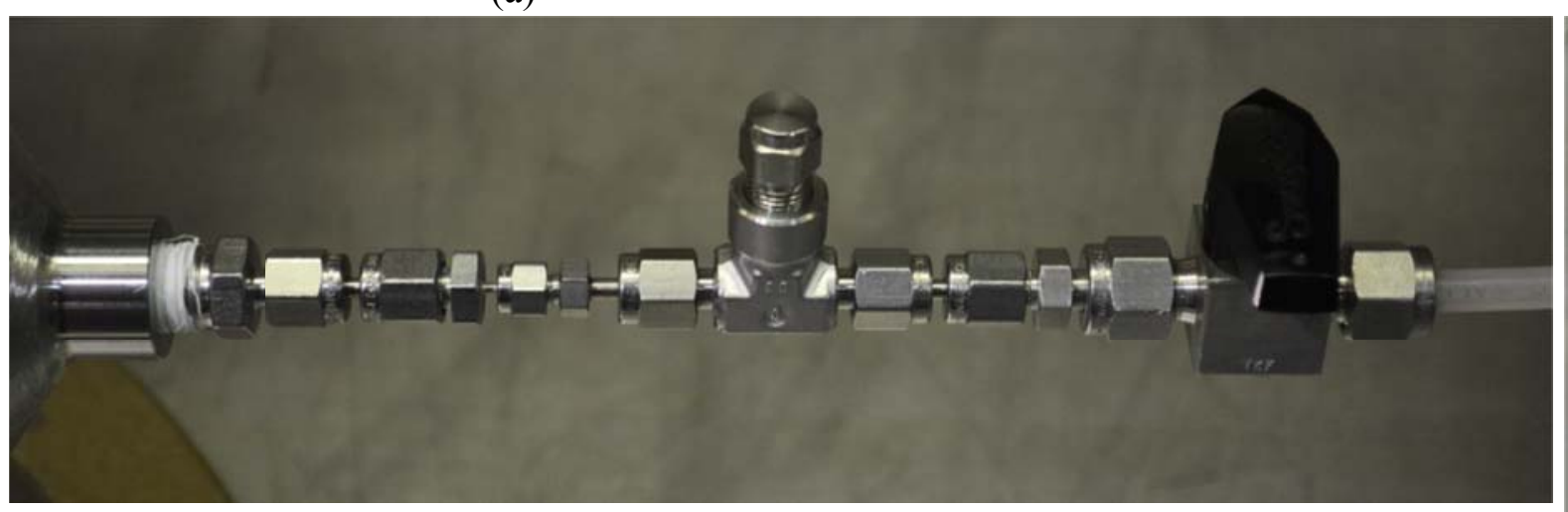

(c)

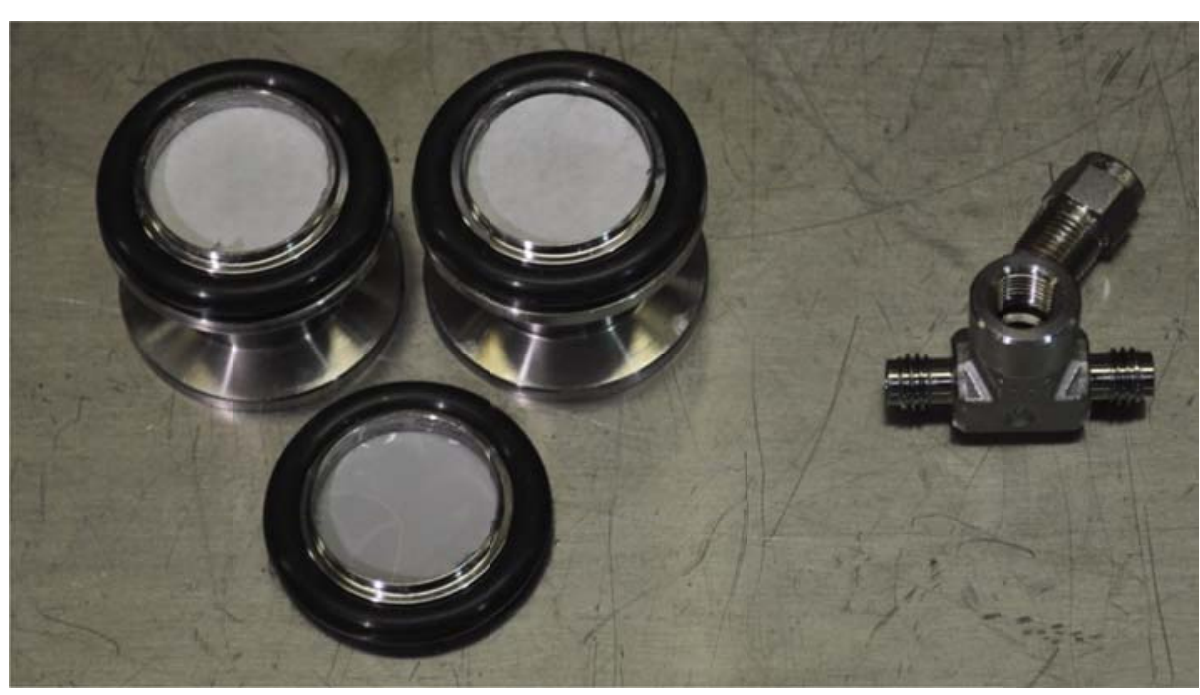

(b)

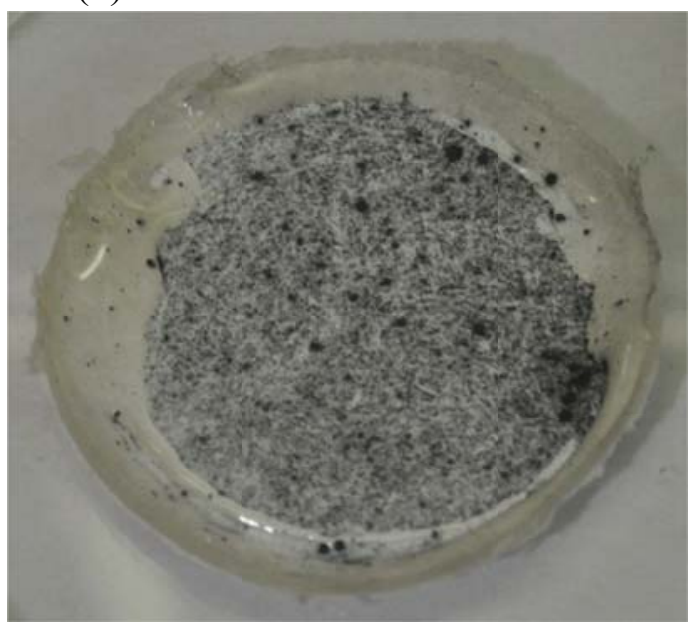

(d)

Figure 50: Photographs of ATS components. 3-stage filters mounted in vacuum fittings are shown in (a) and (b). A loading tee is shown in (b) and mounted in downstream of the isolation valve in (c). A sample $5 \mu \mathrm{m}$ pre-filter with tungsten carbide is shown in (d). 


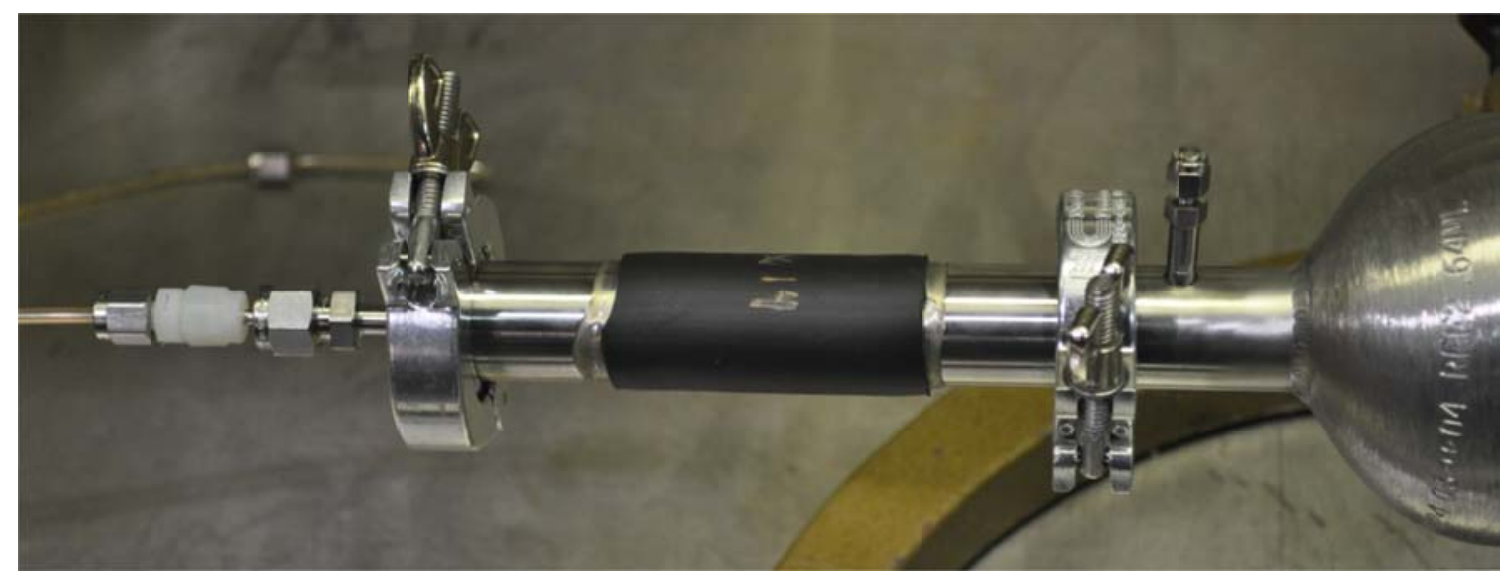

(a)

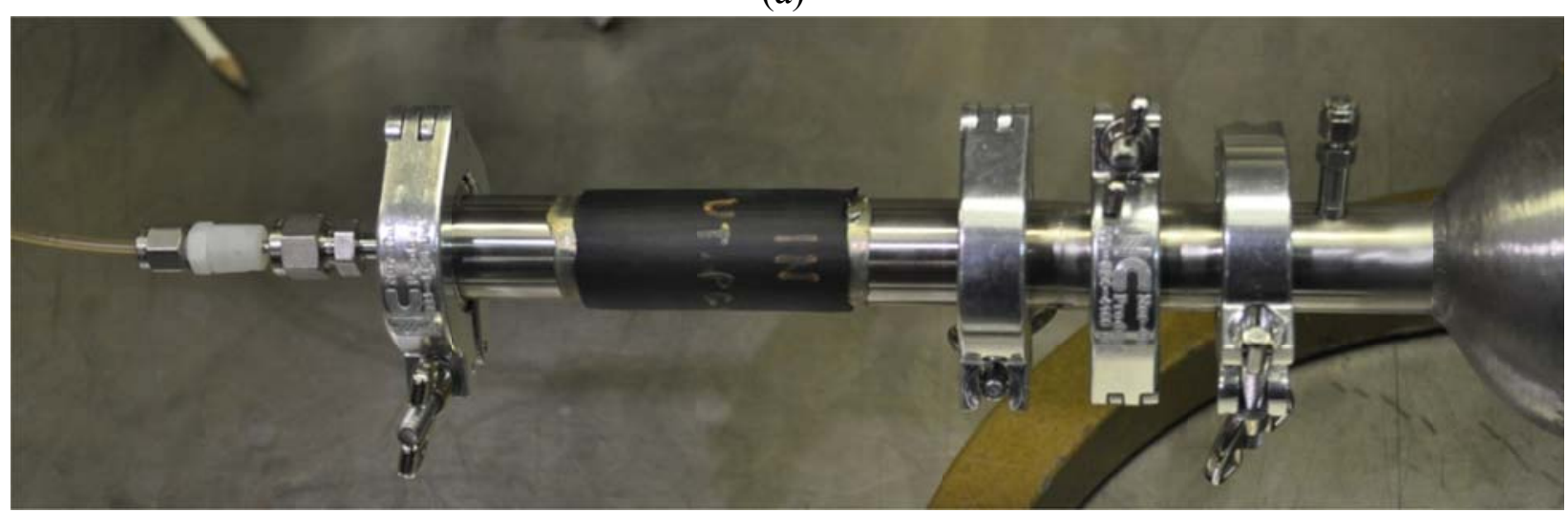

(b)

Figure 51: Photograph of potted graphite with pre-filter (a) and 3-stage filters (b). 
PNNL-21014

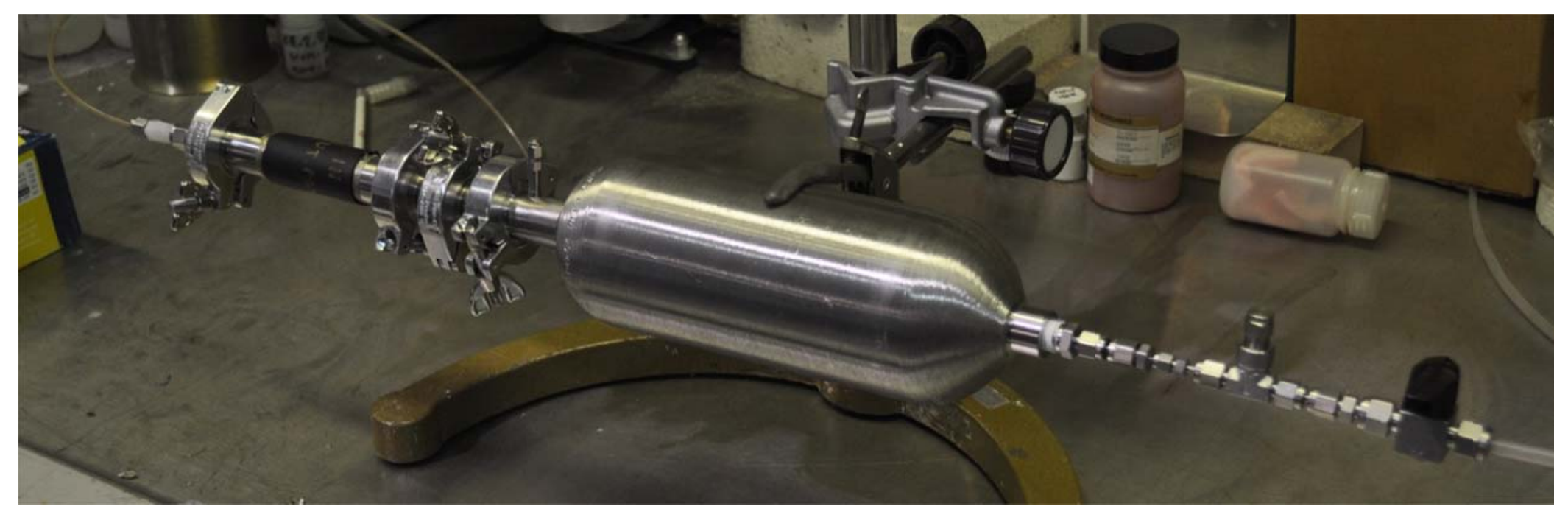

Figure 52: Photograph of Phase I ATS. 


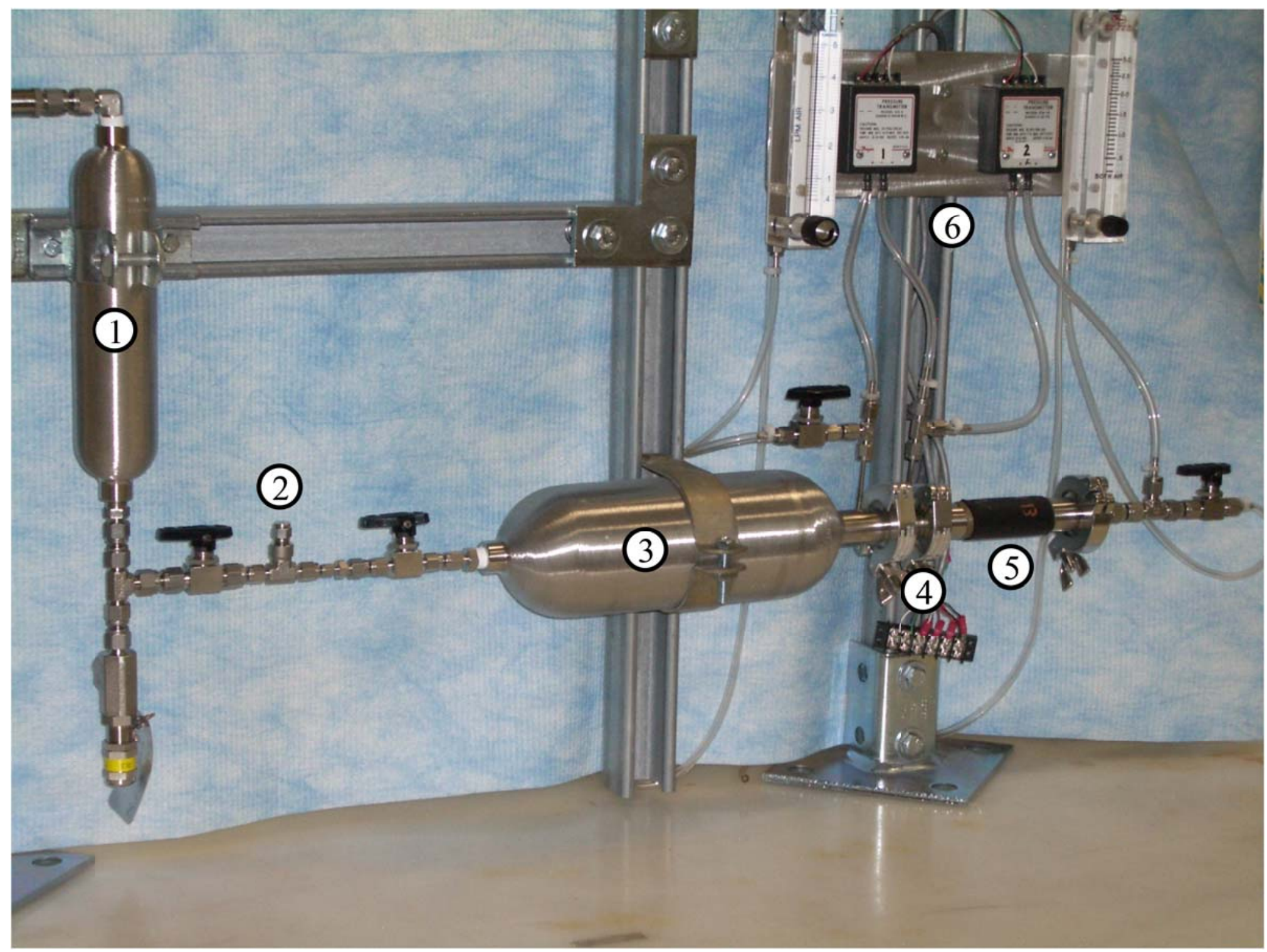

Figure 53: Photograph of radiological environment ATS, where 1 is the pressure chamber, 2 is the loading tee, 3 is the mixing chamber, 4 is the $5 \mu \mathrm{m}$ pre-filter, 5 is the potted graphite sample and 6 is the pressure and flow measurement instruments. 


\section{Appendix F - Review of Carbon Dioxide Oxidation of Uranium}

\section{F.1 Oxidation Experimental Technique}

A Seiko 320 TG/DTA thermogravimetric/differential thermal analyzer was employed to characterize the reaction of uranium with $\mathrm{CO}_{2}$. Uranium metal beads from the stock described by Delegard et al. (2004), and Matheson Tri-Gas Research Purity $\mathrm{CO}_{2}$ and Ultra High Purity neon (compositions provided in Table 14) were utilized. The neon was used with uranium metal to investigate air in-leakage into the TG/DTA, as a sealed system would result in no oxidation, which was measured via the mass gain.

Table 14: Analyzed Composition of Research Grade $\mathrm{CO}_{2}$

\begin{tabular}{|c|c|c|c|}
\hline \multicolumn{2}{|c|}{ Research Purity $\mathrm{CO}_{2}$} & \multicolumn{2}{c|}{ Ultra High Purity Ne } \\
\hline Constituent & Certified Concentration & Constituent & $\begin{array}{l}\text { Specification } \\
\text { Concentration }\end{array}$ \\
\hline $\mathrm{CO}_{2}$ & $99.999 \%$ & $\mathrm{Ne}$ & $99.999 \%$ \\
\hline $\mathrm{CO}$ & $\mathrm{ND}(<0.1 \mathrm{vppm})$ & $\mathrm{Ar}$ & $<1 \mathrm{vppm}$ \\
\hline $\mathrm{H}_{2}$ & $\mathrm{ND}(<0.1 \mathrm{vppm})$ & $\mathrm{CO}_{2}$ & $<1 \mathrm{vppm}$ \\
\hline $\mathrm{O}_{2}$ & $0.09 \mathrm{vppm}$ & $\mathrm{CO}$ & $<1 \mathrm{vppm}$ \\
\hline $\mathrm{THC}^{2}$ & $0.88 \mathrm{vppm}$ & $\mathrm{N}_{2}$ & $<4 \mathrm{vppm}$ \\
\hline $\mathrm{H}_{2} \mathrm{O}$ & $\mathrm{ND}(<0.05 \mathrm{vppm}$ & $\mathrm{THC}$ & $<1 \mathrm{vppm}$ \\
\hline \multicolumn{2}{|r|}{} & $\mathrm{H}_{2} \mathrm{O}$ & $<5 \mathrm{vppm}$ \\
\hline
\end{tabular}

Simultaneous TG/DTA measures the mass and relative temperature changes of a sample as it is exposed to a controlled atmosphere during sample heating or cooling at a controlled rate or under isothermal conditions. Mass change provides a measure of the amount of oxygen or carbon added to the uranium in the case of $\mathrm{CO} 2$ or $\mathrm{CO}$ reaction with $\mathrm{U}$ metal. DTA measures whether a reaction is endothermic (requires heat) or exothermic (produces heat).

Initial experiments indicated substantial air in-leakage into the purge gas system. Intensive effort was spent to eliminate the air in-leakage. The system was purged with helium (He) and a highly sensitive $\mathrm{He}$ leak detector was used to identify leaks from the TG/DTA. The experimental results presented below were performed after no further leaks were observed.

According to Delegard et al. (2004), the uranium metal particles used were typically spherical beads (see Figure 54) with diameters ranging from 200 to $1100 \mu \mathrm{m}$ (refer to Figure 55). The uranium content, based on spectrochemical analysis, was 99.7, with un-quantified traces of iron and aluminum based on energy dispersive spectrometric (EDS) analysis performed during scanning electron microscopic (SEM) analysis. The metal beads, as shown in Figure 54, had a thin coating of oxide. The beads were cleaned by treatment 
with an ammonium carbonate solution and hydrogen peroxide $(10 \mathrm{~mL}$ saturated ammonium carbonate, 1 $\mathrm{mL} 30 \% \mathrm{H}_{2} \mathrm{O}_{2}$ ). The beads were then rinsed with a dilute ammonium carbonate solution, which was replaced with ethanol. The ethanol was then exchanged with fresh ethanol. The ethanol coated beads were then placed into the TG/DTA and dried in the purge gas $\mathrm{CO}_{2}$ or Ne. This provided a silver-color, oxide-free uranium metal for investigation.

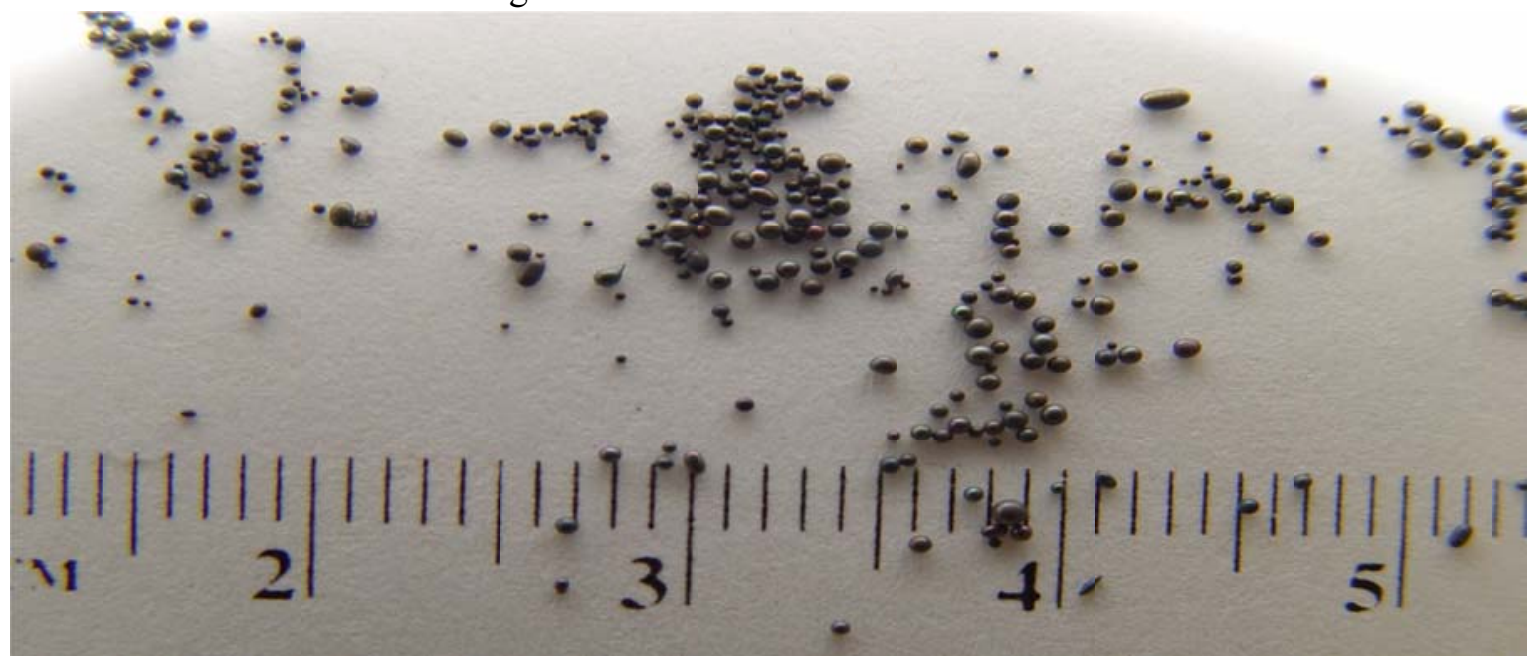

Figure 54: Uranium metal beads, where the scale is in $\mathrm{cm}$ (Delegard et al. 2004).

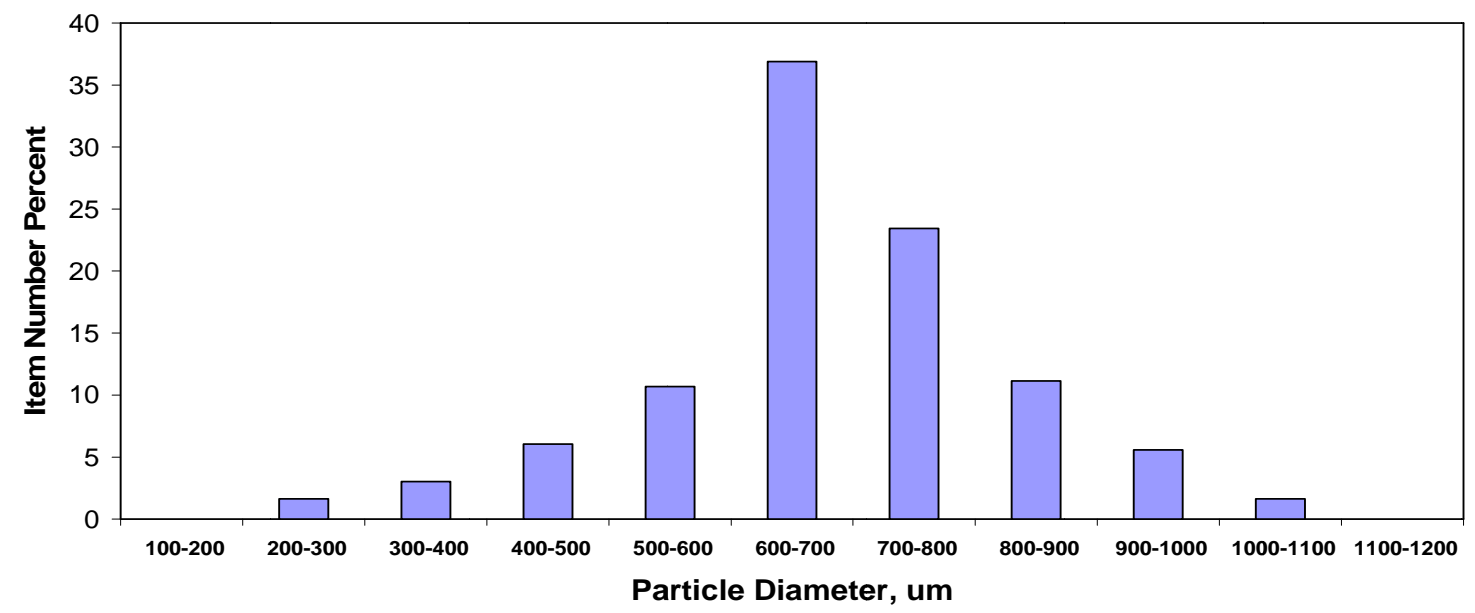

Figure 55: Particle size distribution of uranium metal beads (Delegard et al. 2004).

\section{F.2 Preparation of $\mathrm{CO}_{2}$-Oxidized Uranium Metal}

Upon sealing the TG/DTA and observing no further leaks, two experiments were performed to determine; first, how well the system prevented air ingress by exposing $\mathrm{U}$ metal to $\mathrm{Ne}$ and, second, exposing $\mathrm{U}$ metal to $\mathrm{CO}_{2}$ to quantify oxidation. The results of these two experiments are presented in Figure 56 . In the $\mathrm{Ne}$ experiment, the sample was heated to $480^{\circ} \mathrm{C}$ and maintained for $30 \mathrm{~min}$. In the $\mathrm{CO}_{2}$ experiment, the $\mathrm{U}$ was heated to $580^{\circ} \mathrm{C}$ and held at constant temperature for 30 minutes.

The formula provided by Pearce (1989) for $\mathrm{CO}_{2}$ oxidation of $\mathrm{U}$ metal is 
PNNL-21014

$$
\log \mathrm{k}=6.746-5602 / \mathrm{T}
$$

where the reaction rate $\mathrm{k}$ is in units of $\mathrm{mg} /\left(\mathrm{cm}^{2}-\mathrm{h}\right)$ and $\mathrm{T}$ is temperature in ${ }^{\circ} \mathrm{C}$. The equations provided by Pearce for $\mathrm{O} 2$ oxidation of $\mathrm{U}$ metal are

$$
\log \mathrm{k}=8.9464-4638.2 / \mathrm{T}\left(\mathrm{T}<300^{\circ} \mathrm{C}\right)
$$

and

$$
\log \mathrm{k}=28.381-7 \log \mathrm{T}-4638.2 / \mathrm{T}\left(\mathrm{T}>300^{\circ} \mathrm{C}\right)
$$

Based on Eqn. (6.7), the reaction rate at $577^{\circ} \mathrm{C}$ for $\mathrm{CO}_{2}$ oxidation of $\mathrm{U}$ metal will be $1.43 \mathrm{mg} /\left(\mathrm{cm}^{2}-\mathrm{h}\right)$, which is comparable to the $1.5 \mathrm{mg} /\left(\mathrm{cm}^{2}-\mathrm{h}\right)$ rate measured in the following experiment. This indicates that efforts were successful in eliminating the significant air in-leakage into the TG/DTA or that the airin-leakage is sufficient to provide a rate nearly equivalent to that for $100 \% \mathrm{CO}_{2}$. The similarity in rates for the $\mathrm{Ne}$ and $\mathrm{CO}_{2}$ experiments suggests that the latter reason might be the explanation.

The $\mathrm{CO}_{2}$-oxidization of $\mathrm{U}$ experiment was continued by heating the $\mathrm{U}$ metal to $580^{\circ} \mathrm{C}$ and holding it isothermally there for another 1,000 min as shown in Figure 57. The reaction rate continued as presented in Figure 56.

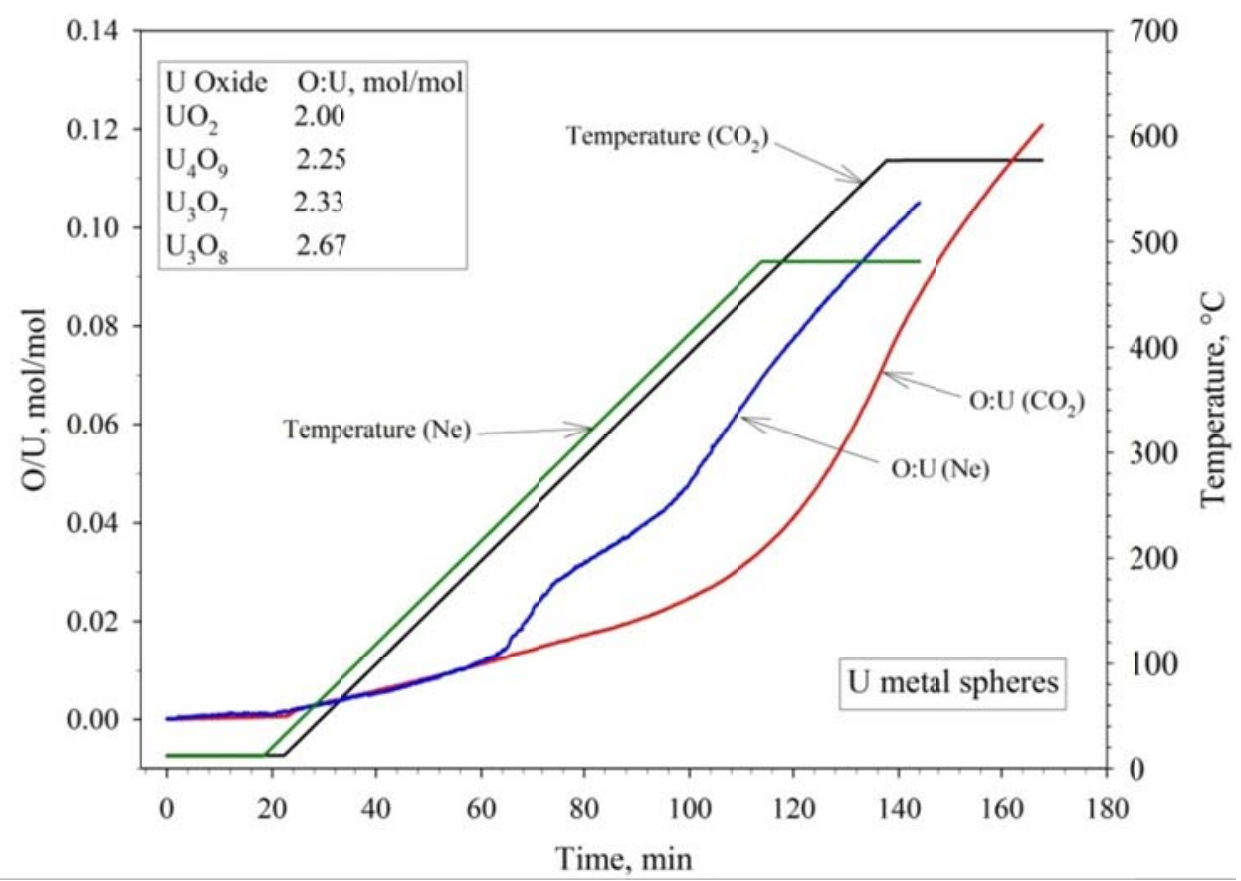

Figure 56: Observed oxidation behavior of U metal in TG/DTA experiments 
PNNL-21014

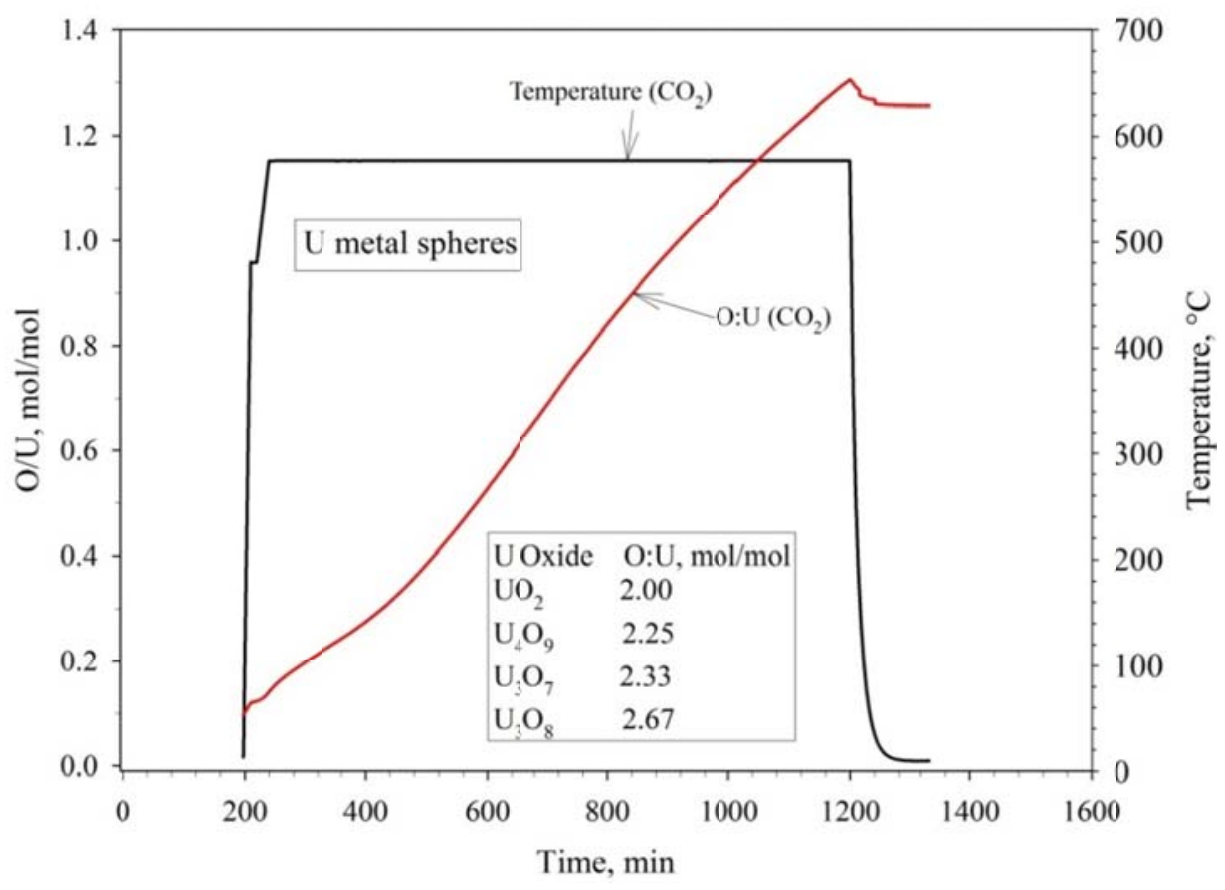

Figure 57: Continued $\mathrm{CO}_{2}$ oxidation of $\mathrm{U}$ metal experiment isothermally at $580^{\circ} \mathrm{C}$

Although some success was had with the preparation of $\mathrm{CO}_{2}$-oxidized $\mathrm{UO}_{2}$, there was insufficient time to assure that the reaction was prototypic and generate substantial mass that could be analyzed and deployed in the ATS testing. Therefore, recycled Redding $\mathrm{EU} \mathrm{UO}_{2}$ was obtained to be used for aerosol transport testing. The specifications of this material are provided in section 4.2.3. 


\section{Appendix G - Unit Collector Model Details}

Single collector capture efficiency by the diffusion mechanism was estimated using

$$
\eta_{D}=4.0 A_{s}{ }^{1 / 3}\left(\frac{1}{P e}\right)^{2 / 3},
$$

where $A_{S}$ is a geometric parameter, which accounts for the effect of neighboring collectors in a packed bed on the flow field (Elimelech et al., 1995), and was calculated from

$$
A_{S}=\frac{2\left(1-p^{5}\right)}{2-3 p+3 p^{5}-2 p^{6}}
$$

where

$$
p=(1-\varepsilon)^{1 / 3},
$$

and $\varepsilon$ is void fraction or porosity.

The particle Peclet number, Pe, is given by

$$
P e=\frac{u_{s} \cdot d_{c}}{D_{p}},
$$

where $u_{s}$ is the superficial velocity through the porous material, $d_{c}$ is the collector diameter, and $D_{p}$ is the particle diffusivity, which is estimated using

$$
D_{p}=\frac{k_{B} \cdot T \cdot S C F}{3 \cdot \pi \cdot \mu \cdot d_{p}},
$$

where $k_{B}$ is Boltzmann's constant, $\mu$ is the dynamic viscosity, and $T$ is the absolute temperature (Konstandopoulos et al., 2002).

The Stokes-Cunningham factor, SCF, represents an interpolation to cover diffusion behavior in between the viscous and free molecular regimes and is calculated using

$$
S C F=1+K n\left(1.257+0.4 e^{-1.1 / K n}\right)
$$

where $\mathrm{Kn}$ is the Knudsen number ( Konstandopoulos et al., 2002) (Friedlander, 2000). The Knudsen number is given by.

$$
K n=\frac{2 \lambda}{d_{p}},
$$

where $\lambda$ is the gas mean free path, calculated with 


$$
\lambda=v \sqrt{\frac{\pi \cdot M W}{2 R \cdot T}},
$$

where $v$ is the fluid kinematic viscosity, $M W$ is the fluid molecular weight, and $R$ is the universal gas constant.

Single collector capture efficiency by interception was estimated using

$$
\eta_{I}=\frac{3}{2} A_{S} R^{2},
$$

where $R$ is the ratio of particle diameter to collector diameter (Elimelech et al., 1995).

Collector capture efficiency by sedimentation or gravity settling was estimated using

$$
\eta_{G}=\frac{\rho_{p}-\rho}{18 \mu \cdot u_{s}} g d_{p}^{2},
$$

where $\rho$ is the fluid density, $\rho_{p}$ is the aerosol particle density, and $g$ is acceleration due to gravity (Elimelech et al., 1995).

Capture efficiency by momentum or inertial impaction was estimated using

$$
\eta_{M}=\frac{S t k_{a d j}^{3}}{0.014+S t k_{a d j}^{3}},
$$

where the adjusted Stokes parameter, Stk $\mathrm{adj}_{j}$, is calculated according to (Otani et al., 1989)

$$
S t k_{a d j}=\left(1+\frac{1.75 \operatorname{Re} \varepsilon}{150(1-\varepsilon)}\right) S t k .
$$

The Reynolds number, Re, based on the collector diameter is given by

$$
\operatorname{Re}=\frac{u_{i} \cdot d_{p}}{v} .
$$

The conventional Stokes number, Stk, is given by

$$
S t k=\frac{u_{i}\left(\rho_{p}-\rho\right) d_{p}{ }^{2} \cdot S C F}{9 \mu \cdot d_{c}},
$$

where $u_{i}$ is the interstitial velocity, which is defined by

$$
u_{i}=\frac{u_{s}}{\varepsilon} \text {. }
$$

The overall collector efficiency is approximated as the sum of the capture efficiencies by all of the relevant mechanisms (Elimelech et al., 1995)

$$
\eta_{c}=\eta_{D}+\eta_{I}+\eta_{G}+\eta_{M} .
$$


The concentration of aerosol particles of a given size penetrating into the porous medium decays exponentially with depth according to

$$
\ln \left(\frac{C}{C_{0}}\right)=-\frac{3}{4} \frac{(1-\varepsilon) \eta_{c} L}{r_{c}}
$$

where $C_{0}$ is the concentration entering the filter, $C$ is the concentration penetrating to a given depth, $L$, and $r_{c}$ is the collector radius (Elimelech et al., 1995).

Filter efficiency predictions are very sensitive to the collector diameter, $d_{c}$. For a packed bed of spherical grains, this is simply the grain diameter. When a unit collector model is used to approximate the filtration behavior of another porous medium, an equivalent collector diameter must be estimated. One approach is to estimate the collector diameter from the sphere diameter of a packed bed having the same equivalent pore size as the medium of interest. In a packed bed of spheres, the sphere diameter, $\mathrm{d}_{\mathrm{sph}}$, is related to a representative average pore diameter by

$$
d_{s p h}=d_{\text {pore }} \frac{6}{4} \frac{(1-\varepsilon)}{\varepsilon} .
$$

The equivalent pore diameter is considered to be four times the hydraulic radius, based on fluid volume and wetted surface area (Geankoplis, 1993), which could be approximated by an value obtained using a technique such as mercury porosimetry.

Another approach is to estimate the sphere diameter using the permeability of the medium. This can be done using the Blake-Kozeny equation (Geankoplis, 1993) for laminar flow through a bed of spherical particles, given by

$$
d_{s p h}=\sqrt{150 k \frac{(1-\varepsilon)^{2}}{\varepsilon^{3}}},
$$

where $k$ is the permeability. 



\section{\# Internal Distribution}

Pacific Northwest National Laboratory

P.O. Box 999

Richland, WA 99352

J Blanchard

K7-15

RD Scheele

P7-25

ML Stewart

K7-15

BD Reid

K8-34

PA Gauglitz

K7-15

LM Bagaasen

K6-28 